Solução numérica de escoamentos viscoelásticos tridimensionais com superfícies livres: Fluidos de Segunda Ordem 
SERVIÇO DE PÓS-GRADUAÇÃO DO ICMC-USP

Data de Depósito:

Assinatura:

\title{
Solução numérica de escoamentos viscoelásticos tridimensionais com superfícies livres: Fluidos de Segunda Ordem
}

\author{
Igor Feliciano Simplicio Revoredo
}

Orientador: Prof. Dr. Murilo Francisco Tomé

Dissertação apresentada ao Instituto de Ciências Matemáticas e de Computação - ICMC-USP, como parte dos requisitos para obtenção do título de Mestre em Ciências - Ciências de Computação e Matemática Computacional.

USP - São Carlos

Fevereiro/2010 
Aos meus pais,

Fátima e José 



\section{Agradecimentos}

Primeiramente a Deus, pelo dom da vida, por seu amor e orientação.

Aos meus pais, em especial a minha mãe Fátima, pelo amor, apoio e confiança sempre expressados e pelas correções nos momentos certos.

Às minhas irmãs Gleyce e Camila, pelo carinho e incentivo de toda hora.

À minha noiva Tânia, pelo amor, companhia e ajuda durante estes anos do mestrado.

Ao professor Dr. Murilo Francisco Tomé do ICMC-USP, pela orientação e tranquilidade passada nos momentos desafiadores.

Aos amigos Fernando, Rafael, Pedro, Fernando Kurokawa, Alysson, do LCAD, pela atenção e colaboração.

Aos amigos de Teresina, Ronildo, Júnior, Ricardo, Aldir e outros que incentivaram e ajudaram diretamente neste trabalho.

Aos amigos de São Carlos, Reinaldo, Tiago, Adriano, Pelegrine, Oswaldo, Patrícia, Alanna, Nilmara, Sarah, e muitos outros que sempre deram um voto de apoio.

Ao professor Dr. Sergio Luiz Frey da UFRGS e ao professor Dr. Roney Leon Thompson da UFF, pelos comentário e críticas feitos na banca virtual do meu Exame de Qualificação.

Meus sinceros agradecimentos a todos os professores e alunos dos grupos de pesquisa do LCAD e funcionários do ICMC-USP, pela saudável convivência e apoio dado durante a realização deste trabalho.

Por fim, meu agradecimento ao CNPq pelo auxílio financeiro que possibilitou a realização deste trabalho (números dos processos: 131947/2007-9 e 135938/2008-2). 


\section{Resumo}

Este trabalho apresenta uma técnica de diferenças finitas para resolver a equação constitutiva Fluido de Segunda Ordem para escoamentos tridimensionais com superfície livre. As equações governantes são resolvidas pelo método de diferenças finitas em uma malha deslocada 3D. A superfície livre é modelada por células marcadoras (Marker-and-Cell) e as condições de contorno a superfície livre são empregadas. O método numérico apresentado neste trabalho foi validado pela comparação entre as soluções numéricas obtidas para o escoamento em um tubo com a solução analítica correspondente para Fluidos de Segunda Ordem. Ao fazer refinamento de malha, a convergência do método numérico foi verificada. Resultados numéricos da simulação do problema do inchamento do extrudado para números de Deborah $D e \leq 0.3$ são apresentados.

Palavras-Chave: Diferenças finitas, Escoamentos viscoelásticos, Marker-and-Cell, Superfícies livres, Fluido de Segunda Ordem. 


\section{Abstract}

This work presents a finite difference method to simulate three-dimensional viscoelastic flow with free surfaces governed by the constitutive equation Second Order Fluid. The governing equations are solved by the finite difference method in a three-dimensional shifted mesh. The free surface of fluid is modeled by the Marker-and-Cell method which allows for the visualization and the location of the free surface of fluid. The full free surface stress conditions are employed. The numerical method developed in this work is validated by comparing the numerical and analytic solutions for the steady state flow of a Second Order Fluid in a pipe. By using mesh refinement convergence results are given. Numerical results of the simulation of the transient extrudate swell of a Second Order Fluid of the Deborah number $D e \leq 0.3$ are presented.

Keywords: Finite difference, Viscoelastic flows, Marker-and-Cell, Free surface, Seconde Order Fluid. 



\section{Sumário}

$\begin{array}{ll}\text { Agradecimentos } & \text { i }\end{array}$

Resumo

Abstract $\quad$ v

$\begin{array}{ll}\text { Nomenclatura } & \text { Xv }\end{array}$

Introdução $\quad$ xvii

1 Equações governantes $\quad 1$

1.1 Obtenção da derivada convectada contravariante em coordenadas cartesianas 3

1.2 Obtenção das equações governantes em coordenadas cartesianas . . . . . . . . 6

1.3 Adimensionalização das equações governantes . . . . . . . . . . . . . 8

1.4 Condições de contorno nos injetores, ejetores e contornos rígidos . . . . . . . 9

1.5 Condições de contorno nas superfícies livres . . . . . . . . . . . . . . . . 9

2 Método numérico utilizado $\quad 11$

2.1 GENSMAC3D-SOF . . . . . . . . . . . . . . . . . . . . 11

2.2 Algoritmo computacional . . . . . . . . . . . . . . . . . . . 11

3 Aproximação por diferenças finitas $\quad \mathbf{1 3}$

3.1 Discretização da equação de conservação de quantidade de movimento . . . . 13

3.1.1 Aproximações de segunda ordem . . . . . . . . . . . . . . . 15

3.2 Discretização da equação de Poisson . . . . . . . . . . . . . . . . . . . . . 15

3.3 Velocidades finais . . . . . . . . . . . . . . . . . . . 16

3.4 Discretização da função reológica $\Phi$. . . . . . . . . . . . . . . . . . . . 16

3.4.1 Aproximações de segunda ordem . . . . . . . . . . . . . . . . 18

3.5 Aplicação das condições de contorno na superfície livre . . . . . . . . . . . . 18

3.5.1 Superfície plana paralela a um eixo de coordenadas . . . . . . . . . . 18

3.5.2 Superfície plana com inclinação de $45^{\circ}$. . . . . . . . . . . . . . . 20

3.5.3 Superfície plana com inclinação de $60^{\circ}$. . . . . . . . . . . . . . . . . 22

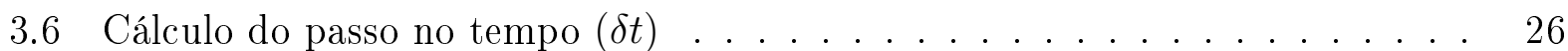

4 Validação do método numérico $\quad 27$

4.1 Escoamento totalmente desenvolvido em um tubo . . . . . . . . . . . . . 27 
6 Conclusão e considerações finais

A Discretização da função reológica $\Phi$

A.1 Componente $\Phi^{x y}$ discretizada no centro da célula . . . . . . . . . . . . . . 43

A.2 Componente $\Phi^{x z}$ discretizada no centro da célula . . . . . . . . . . . . . . . . 43

A.3 Componente $\Phi^{y y}$ discretizada no centro da célula . . . . . . . . . . . . . 44

A.4 Componente $\Phi^{y z}$ discretizada no centro da célula . . . . . . . . . . . . . . . . 44

A.5 Componente $\Phi^{z z}$ discretizada no centro da célula . . . . . . . . . . . . . . . . 44

B Tratamento de superfícies planas consideradas no código FreeFlow3D 45

B.1 Superfície plana paralela a um eixo de coordenadas . . . . . . . . . . . 45

B.1.1 Face $\left(i+\frac{1}{2}\right)$, onde $n=(1,0,0), m 1=(0,1,0)$ e $m 2=(0,0,1) \ldots \ldots$

B.1.2 Face $\left(i-\frac{1}{2}\right)$, onde $n=(-1,0,0), m 1=(0,1,0)$ e $m 2=(0,0,1) \ldots . \quad 46$

B.1.3 Face $\left(j+\frac{1}{2}\right)$, onde $n=(0,1,0), m 1=(1,0,0)$ e $m 2=(0,0,1) \ldots . \quad 47$

B.1.4 Face $\left(j-\frac{1}{2}\right)$, onde $n=(0,-1,0), m 1=(1,0,0)$ e $m 2=(0,0,1) \ldots . \quad 48$

B.1.5 Face $\left(k-\frac{1}{2}\right)$, onde $n=(0,0,-1), m 1=(0,1,0)$ e $m 2=(1,0,0) \ldots . \quad 49$

B.2 Superfície plana com inclinação de $45^{\circ} \ldots \ldots \ldots$. . . . . . . . . 50

B.2.1 Células $\mathrm{S}$ possuem apenas a face $\left(i+\frac{1}{2}\right)$ e $\left(j+\frac{1}{2}\right)$ em contato com células E. Onde $n=\left(\frac{\sqrt{2}}{2}, \frac{\sqrt{2}}{2}, 0\right), m 1=\left(\frac{\sqrt{2}}{2},-\frac{\sqrt{2}}{2}, 0\right)$ e $m 2=(0,0,1)$.

B.2.2 Células $\mathrm{S}$ possuem apenas a face $\left(j+\frac{1}{2}\right)$ e $\left(k+\frac{1}{2}\right)$ em contato com células E. Onde $n=\left(0, \frac{\sqrt{2}}{2}, \frac{\sqrt{2}}{2}\right), m 1=\left(0, \frac{\sqrt{2}}{2},-\frac{\sqrt{2}}{2}\right)$ e $m 2=(1,0,0)$.

B.2.3 Células $\mathrm{S}$ possuem apenas a face $\left(i-\frac{1}{2}\right)$ e $\left(j+\frac{1}{2}\right)$ em contato com células E. Onde $n=\left(-\frac{\sqrt{2}}{2}, \frac{\sqrt{2}}{2}, 0\right), m 1=\left(\frac{\sqrt{2}}{2}, \frac{\sqrt{2}}{2}, 0\right)$ e $m 2=(0,0,1)$.

B.2.4 Células $\mathrm{S}$ possuem apenas a face $\left(i-\frac{1}{2}\right)$ e $\left(k+\frac{1}{2}\right)$ em contato com células E. Onde $n=\left(-\frac{\sqrt{2}}{2}, 0, \frac{\sqrt{2}}{2}\right), m 1=\left(\frac{\sqrt{2}}{2}, 0, \frac{\sqrt{2}}{2}\right)$ e $m 2=(0,1,0)$.

B.2.5 Células $\mathrm{S}$ possuem apenas a face $\left(i+\frac{1}{2}\right)$ e $\left(j-\frac{1}{2}\right)$ em contato com células E. Onde $n=\left(\frac{\sqrt{2}}{2},-\frac{\sqrt{2}}{2}, 0\right), m 1=\left(\frac{\sqrt{2}}{2}, \frac{\sqrt{2}}{2}, 0\right)$ e $m 2=(0,0,1)$.

B.2.6 Células $\mathrm{S}$ possuem apenas a face $\left(j-\frac{1}{2}\right)$ e $\left(k+\frac{1}{2}\right)$ em contato com células E. Onde $n=\left(0,-\frac{\sqrt{2}}{2}, \frac{\sqrt{2}}{2}\right), m 1=\left(0, \frac{\sqrt{2}}{2}, \frac{\sqrt{2}}{2}\right)$ e $m 2=(1,0,0)$.

B.2.7 Células $\mathrm{S}$ possuem apenas a face $\left(i-\frac{1}{2}\right)$ e $\left(j-\frac{1}{2}\right)$ em contato com células E. Onde $n=\left(-\frac{\sqrt{2}}{2},-\frac{\sqrt{2}}{2}, 0\right), m 1=\left(\frac{\sqrt{2}}{2},-\frac{\sqrt{2}}{2}, 0\right)$ e $m 2=(0,0,1) . \quad 57$

B.2.8 Células $\mathrm{S}$ possuem apenas a face $\left(i+\frac{1}{2}\right)$ e $\left(k-\frac{1}{2}\right)$ em contato com células E. Onde $n=\left(\frac{\sqrt{2}}{2}, 0,-\frac{\sqrt{2}}{2}\right), m 1=\left(\frac{\sqrt{2}}{2}, 0, \frac{\sqrt{2}}{2}\right)$ e $m 2=(0,1,0)$.

B.2.9 Células $\mathrm{S}$ possuem apenas a face $\left(j+\frac{1}{2}\right)$ e $\left(k-\frac{1}{2}\right)$ em contato com células E. Onde $n=\left(0, \frac{\sqrt{2}}{2},-\frac{\sqrt{2}}{2}\right), m 1=\left(0, \frac{\sqrt{2}}{2}, \frac{\sqrt{2}}{2}\right)$ e $m 2=(1,0,0)$.

B.2.10 Células $\mathrm{S}$ possuem apenas a face $\left(i-\frac{1}{2}\right)$ e $\left(k-\frac{1}{2}\right)$ em contato com células E. Onde $n=\left(-\frac{\sqrt{2}}{2}, 0,-\frac{\sqrt{2}}{2}\right), m 1=\left(\frac{\sqrt{2}}{2}, 0,-\frac{\sqrt{2}}{2}\right)$ e $m 2=(0,1,0) . \quad 60$

B.2.11 Células $\mathrm{S}$ possuem apenas a face $\left(j-\frac{1}{2}\right)$ e $\left(k-\frac{1}{2}\right)$ em contato com células E. Onde $n=\left(0,-\frac{\sqrt{2}}{2},-\frac{\sqrt{2}}{2}\right), m 1=\left(0, \frac{\sqrt{2}}{2},-\frac{\sqrt{2}}{2}\right)$ e $m 2=(1,0,0) . \quad 61$

B.3 Superfície plana com inclinação de $60^{\circ} \ldots \ldots \ldots$. . . . . . . . 63

B.3.1 Células $\mathrm{S}$ possuem as faces $\left(i-\frac{1}{2}\right),\left(j+\frac{1}{2}\right)$ e $\left(k+\frac{1}{2}\right)$ em contato com células E. . . . . . . . . . . . . . . . . .

B.3.2 Células $\mathrm{S}$ possuem as faces $\left(i+\frac{1}{2}\right),\left(j-\frac{1}{2}\right)$ e $\left(k+\frac{1}{2}\right)$ em contato com células E. 
B.3.3 Células $\mathrm{S}$ possuem as faces $\left(i-\frac{1}{2}\right),\left(j-\frac{1}{2}\right)$ e $\left(k+\frac{1}{2}\right)$ em contato com células E. . . . . . . . . . . . . . . . . . .

B.3.4 Células S possuem as faces $\left(i+\frac{1}{2}\right),\left(j+\frac{1}{2}\right)$ e $\left(k-\frac{1}{2}\right)$ em contato com células E. . . . . . . . . . . . . . . . . . .

Células S possuem as faces $\left(i-\frac{1}{2}\right),\left(j+\frac{1}{2}\right)$ e $\left(k-\frac{1}{2}\right)$ em contato com células E. . . . . . . . . . . . . . . . . . . 75

B.3.6 Células $\mathrm{S}$ possuem as faces $\left(i+\frac{1}{2}\right),\left(j-\frac{1}{2}\right)$ e $\left(k-\frac{1}{2}\right)$ em contato com células E. . . . . . . . . . . . . . . . . . . . 78

B.3.7 Células $\mathrm{S}$ possuem as faces $\left(i-\frac{1}{2}\right),\left(j-\frac{1}{2}\right)$ e $\left(k-\frac{1}{2}\right)$ em contato com células E. . . . . . . . . . . . . . . . . . . 


\section{Lista de Figuras}

3.1 Célula (a) e domínio computacional (b) utilizadas pelo Freeflow3D. . . . . . 14

3.2 Pontos utilizados para o cálculo de $\left.\frac{\partial \Phi^{x y}}{\partial y}\right|_{i+\frac{1}{2}, j, k}$ por diferença de segunda ordem. 16

3.3 Pontos utilizados para o cálculo de $\left.\frac{\partial u}{\partial y}\right|_{i, j, k} \ldots \ldots \ldots \ldots \ldots$. . . . . . . . . 18

3.4 Células de superfície com somente uma face em contato com células vazias. . 19

3.5 Célula $\mathrm{S}$ com somente a face $\left(k+\frac{1}{2}\right)$ em contato com células E. . . . . . . . 20

3.6 Células de superfície com duas faces adjacentes em contato com faces de células vazias. . . . . . . . . . . . . . . . . . . 21

3.7 Célula $\mathrm{S}$ com as faces $\left(i+\frac{1}{2}\right)$ e $\left(k+\frac{1}{2}\right)$ em contato com faces de células vazias. 22

3.8 Células de superfície com três faces em contato com células vazias. . . . . . . 23

3.9 Célula $\mathrm{S}$ com as faces $\left(i+\frac{1}{2}\right),\left(j+\frac{1}{2}\right)$ e $\left(k+\frac{1}{2}\right)$ em contato com faces de células vazias. . . . . . . . . . . . . . . . . . 24

4.1 Representação do escoamento em um tubo. . . . . . . . . . . . . . . . . . . . 27

4.2 Enchimento do tudo com fluido de segunda ordem: $D e=0.4, \kappa=0.4$ e malha M2. . . . . . . . . . . . . . . . . . 29

4.3 Visualização de $w(x, y)$ no meio e ao longo do tubo em $t=50 . . .30$

4.4 Comparação entre as soluções analíticas e as soluções numéricas para $w(x, y)$, $\Phi^{x x}, \Phi^{x y}, \Phi^{y y}, \Phi^{z z}$. Coluna da esquerda: malha M0. Coluna do meio: malha M1. Coluna da direita: malha M2. . . . . . . . . . . . . . . . . . 31

4.5 Decaimento dos erros com o refinamento da malha. . . . . . . . . . . . . . 32

5.1 Extrudate swell. . . . . . . . . . . . . . . . . . . . . . 33

5.2 Simulação numérica do inchamento do extrudado para vários números $D e$ e $\kappa$ na malha intermediária (M1). Configuração do fluido nos tempos $t=$ $5,7.5,10,12.5,15 \ldots \ldots \ldots \ldots \ldots \ldots \ldots \ldots \ldots \ldots \ldots \ldots \ldots \ldots \ldots$

5.3 Simulação numérica do inchamento do extrudado na malha mais refinada (M2). Configuração do fluido nos tempos $t=5,7.5,10,12.5,15 \ldots \ldots$. . . 36

B.1 Célula S com somente a face $\left(i+\frac{1}{2}\right)$ em contato com células E. . . . . . . . 45

B.2 Célula $\mathrm{S}$ com somente a face $\left(i-\frac{1}{2}\right)$ em contato com células E. . . . . . . . 46

B.3 Célula $\mathrm{S}$ com somente a face $\left(j+\frac{1}{2}\right)$ em contato com células E. . . . . . . . 47

B.4 Célula $\mathrm{S}$ com somente a face $\left(j-\frac{1}{2}\right)$ em contato com células E. . . . . . . . 48

B.5 Célula $\mathrm{S}$ com somente a face $\left(k-\frac{1}{2}\right)$ em contato com células E. . . . . . . . 49

B.6 Célula $\mathrm{S}$ com as faces $\left(i+\frac{1}{2}\right)$ e $\left(j+\frac{1}{2}\right)$ em contato com faces de células vazias. 50 
B.7 Célula $\mathrm{S}$ com as faces $\left(j+\frac{1}{2}\right)$ e $\left(k+\frac{1}{2}\right)$ em contato com faces de células vazias. 51

B.8 Célula $\mathrm{S}$ com as faces $\left(i-\frac{1}{2}\right)$ e $\left(j+\frac{1}{2}\right)$ em contato com faces de células vazias. 52

B.9 Célula $\mathrm{S}$ com as faces $\left(i-\frac{1}{2}\right)$ e $\left(k+\frac{1}{2}\right)$ em contato com faces de células vazias. 53

B.10 Célula $\mathrm{S}$ com as faces $\left(i+\frac{1}{2}\right)$ e $\left(j-\frac{1}{2}\right)$ em contato com faces de células vazias. 55

B.11 Célula $\mathrm{S}$ com as faces $\left(j-\frac{1}{2}\right)$ e $\left(k+\frac{1}{2}\right)$ em contato com faces de células vazias. 56

B.12 Célula $\mathrm{S}$ com as faces $\left(i-\frac{1}{2}\right)$ e $\left(j-\frac{1}{2}\right)$ em contato com faces de células vazias. 57

B.13 Célula $\mathrm{S}$ com as faces $\left(i+\frac{1}{2}\right)$ e $\left(k-\frac{1}{2}\right)$ em contato com faces de células vazias. 58

B.14 Célula $\mathrm{S}$ com as faces $\left(j+\frac{1}{2}\right)$ e $\left(k-\frac{1}{2}\right)$ em contato com faces de células vazias. 59

B.15 Célula $\mathrm{S}$ com as faces $\left(i-\frac{1}{2}\right)$ e $\left(k-\frac{1}{2}\right)$ em contato com faces de células vazias. 61

B.16 Célula $\mathrm{S}$ com as faces $\left(j-\frac{1}{2}\right)$ e $\left(k-\frac{1}{2}\right)$ em contato com faces de células vazias. 62

B.17 Célula $\mathrm{S}$ com as faces $\left(i-\frac{1}{2}\right),\left(j+\frac{1}{2}\right)$ e $\left(k+\frac{1}{2}\right)$ em contato com faces de células

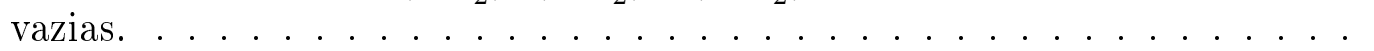

B.18 Célula S com as faces $\left(i+\frac{1}{2}\right),\left(j-\frac{1}{2}\right)$ e $\left(k+\frac{1}{2}\right)$ em contato com faces de células vazias. . . . . . . . . . . . . . . . . . 6 66

B.19 Célula S com as faces $\left(i-\frac{1}{2}\right),\left(j-\frac{1}{2}\right)$ e $\left(k+\frac{1}{2}\right)$ em contato com faces de células

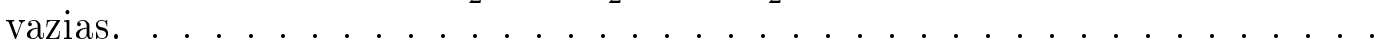

B.20 Célula S com as faces $\left(i+\frac{1}{2}\right),\left(j+\frac{1}{2}\right)$ e $\left(k-\frac{1}{2}\right)$ em contato com faces de células vazias. . . . . . . . . . . . . . . . . . .

B.21 Célula S com as faces $\left(i-\frac{1}{2}\right),\left(j+\frac{1}{2}\right)$ e $\left(k-\frac{1}{2}\right)$ em contato com faces de células vazias. . . . . . . . . . . . . . . . . 76

B.22 Célula S com as faces $\left(i+\frac{1}{2}\right),\left(j-\frac{1}{2}\right)$ e $\left(k-\frac{1}{2}\right)$ em contato com faces de células

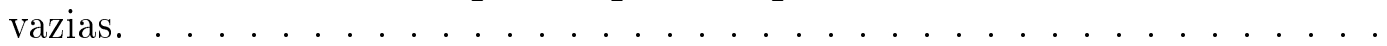

B.23 Célula S com as faces $\left(i-\frac{1}{2}\right),\left(j-\frac{1}{2}\right)$ e $\left(k-\frac{1}{2}\right)$ em contato com faces de células vazias. 


\section{Lista de Tabelas}

4.1 Erros obtidos nas três malhas. . . . . . . . . . . . . . . . . . . . . 30

5.1 Simulação numérica do inchamento do extrudado: taxas de inchamento obtidas. 34 


\section{Nomenclatura}

Propriedades do Fluido

\section{$\rho$}

$\eta_{0}$

\section{Dinâmica do Fluido}

\section{$\mathrm{u}$}

$\mathbf{x}$

$p$

$\sigma$

$\tau$

$t$

$\frac{D \mathbf{D}}{D t}$

\section{Tensores Cinemático}

$\nabla \mathbf{u}$

$\mathrm{D}$

$\stackrel{\nabla}{D}$

$\Phi$

Massa específica do fluido

Coeficiente de viscosidade

(ou viscosidade dinâmica) do fluido

Vetor velocidade $(u, v, w)$

Vetor posição $(x, y, x)$

Campo de pressão

Tensor tensão total $(=-p \mathbf{I}+\boldsymbol{\tau})$

Tensor de tensão extra

Tempo

Derivada material (substancial), quando

o escoamento é incompressível

$\left(=\frac{\partial \mathbf{D}}{\partial t}+\nabla \cdot(\mathbf{u D})\right)$

Tensor gradiente de velocidade $\left(=\frac{\partial u_{j}}{\partial x_{i}}\right)$

Tensor de taxa de deformação

$\left(=\nabla \mathbf{u}+(\nabla \mathbf{u})^{T}\right)$

$\left(=\frac{D(\mathbf{D})}{D t}-\left[(\nabla \mathbf{u})^{T} \cdot \mathbf{D}+\mathbf{D} \cdot \nabla \mathbf{u}\right]\right)$

Tensor não-newtoniano

Derivada convectada contravariante (ou convectada superior)

$\left(=\lambda_{2} \stackrel{\nabla}{\mathbf{D}}+\lambda_{4}(\mathbf{D} \cdot \mathbf{D})\right)$

\section{Geral}

$\mathrm{g}$

Vetor de aceleração gravitacional 


\section{Propriedades Reológicas}

$\lambda_{2}$

$\lambda_{4}$

$N_{1}$

$N_{2}$

$\Psi_{1}$

$\Psi_{2}$

$\tau_{12}$
Primeiro parâmetro reológico

Segundo parâmetro reológico

Primeira diferença de tensões normais

Segunda diferença de tensões normais

Primeiro coeficiente de diferença de tensões normais

Segundo coeficiente de diferença de tensões normais

Tensão de cisalhamento 


\section{Introdução}

Muitos processos na indústria de polímeros envolvem escoamentos com superfícies livres de fluidos complexos. Como exemplo podemos citar a extrusão e o enchimento de containeres com fluidos de reologia complexa. Esses problemas têm motivado o desenvolvimento de métodos numéricos de modo que esses processos possam ser estudados via simulação. Os modelos UCM (Upper-Convected Maxwell), Oldroyd B e PTT (Phan-Thien-Tanner) têm sido considerados por muitos pesquisadores e uma variedade de técnicas para simular escoamentos viscoelásticos governados por esses modelos tem sido desenvolvidos, por exemplo Brasseur et al. [15], Carew et al [16], Huang et al. [33], Marchal e Crochet [21], Phillips e Williams [5], Xue et al. [8], Yoo e Na [10], entre muitos outros pesquisadores. Os problemas investigados têm sido o escoamento através de uma contração 4:1 em duas dimensões (por exemplo, Alves et al. [4], Phillips e Williams [5], Yoo e Na [10]) e em três dimensões (ver por exemplo, Mompean e Deville [22], Xue et al. [8]). O inchamento do extrudado também tem sido estudado por muitos investigadores, por exemplo Brasseur et al. [15], Ryan e Dutta [37], Crochet e Keunings [23, 38], Tomé et al. [20], Paulo et al. [14], entre muitos outros.

Por outro lado, escoamentos viscoelásticos governados pela equação constitutiva Criminale-Ericksen-Filbey (CEF), que é conhecida como Fluido de Segunda Ordem, tem sido pouco estudadas. Entre os trabalhos que simulam escoamentos com superfície livre utilizando essa equação constitutiva, podemos mencionar o trabalho de Gast e Ellington [31] que utilizaram o código FIDAP [30] para simular o inchamento do extrudado de um Fluido de Segunda Ordem e conseguiram resultados para fluidos com pouca elasticidade (isto é, número de Deborah pequeno, $D e \approx 0.1$ ). Mais recentemente, Tomé et al. [13] apresentaram um método numérico para simular escoamentos com superfícies livres governados pela equação constitutiva Fluidos de Segunda Ordem em duas dimensões. Tomé et al. [13] desenvolveram um método de diferenças finitas para resolver as equações governantes e utilizaram o método de partículas marcadoras [32] para representar o fluido. Resultados para o problema da contração plana 4:1 e do inchamento do extrudado para fluidos com alta viscoelasticidade foram obtidos.

O objetivo desse trabalho é estender a técnica apresentada por Tomé et al. [13] para escoamentos tridimensionais. Pretende-se desenvolver uma técnica numérica que seja capaz de simular escoamentos tridimensionais com superfícies livres de Fluidos de Segunda Ordem. Vamos utilizar o método das partículas marcadoras e desenvolver um método de diferenças finitas para resolver as equações básicas. Pretende-se também obter a solução analítica para escoamentos estacionários de Fluidos de Segunda Ordem em um tubo e utilizar essa solução analítica para validar o método numérico proposto nesse trabalho. Resultados numéricos para o problema do inchamento do extrudado são apresentados. 


\section{Organização da dissertação}

O trabalho está organizado da seguinte maneira:

- Capítulo 1: Apresenta as equações governantes para escoamentos viscoelásticos tridimensionais modelados pela equação constitutiva Fluido de Segunda Ordem. Descreve a obtenção da derivada convectada contravariante e das equações governantes em coordenadas cartesianas. Apresenta as equações envolvidas na forma adimensional. Por fim, apresenta as condições de contorno nos injetores, ejetores, contornos rígidos e nas superfícies livres.

- Capítulo 2: Esse capítulo apresenta o algoritmo utilizado para resolver as equações governantes.

- Capítulo 3: Esse capítulo apresenta a discretização das equações básicas (função reológica, equação de conservação de quantidade de movimento, equação de Poisson e velocidades finais) por meio da técnica de diferenças finitas. A aplicação das condições de contorno na superfície livre são também tratadas pelo método de diferenças finitas.

- Capítulo 4: Apresenta os resultados de validação. A solução numérica do escoamento em um tubo é comparada com a respectiva solução analítica. A convergência é demonstrada através do refinamento de malha computacional.

- Capítulo 5: Apresenta os resultados obtidos nas simulações do fenômeno do inchamento do extrudado.

- Capítulo 6: Apresenta as conclusões finais relacionadas à pesquisa desenvolvida. 


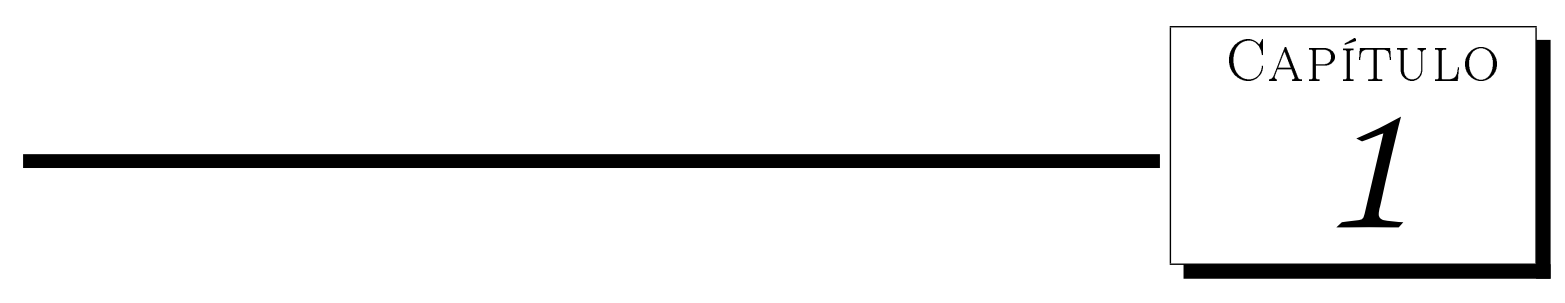

\section{Equações governantes}

As equações que governam o escoamento incompressível de um Fluido de Segunda Ordem (Second Order Fluid - SOF) são, conforme Bird [2], a equação de conservação de quantidade de movimento (1.1), a equação de conservação de massa (continuidade) (1.2) e a equação constitutiva para fluidos de segunda ordem (1.3), como segue:

$$
\begin{gathered}
\rho\left(\frac{\partial \mathbf{u}}{\partial t}+\nabla \cdot(\mathbf{u u})\right)=-\nabla p+\nabla \cdot \boldsymbol{\tau}+\rho \mathbf{g}, \\
\nabla \cdot \mathbf{u}=0 \\
\boldsymbol{\tau}=\eta_{0}\left[\mathbf{D}+\lambda_{2} \stackrel{\nabla}{\mathbf{D}}+\lambda_{4}(\mathbf{D} \cdot \mathbf{D})\right]
\end{gathered}
$$

onde $\mathbf{u}$ é o campo de velocidades, $\rho$ é a massa específica do fluido, $p$ é o campo de pressão, $\mathbf{g}$ é o vetor de aceleração gravitacional, $\tau$ é o tensor de tensão extra. Na equação constitutiva (1.3), $\eta_{0}$ denota a viscosidade e os parâmetros $\lambda_{2}$ e $\lambda_{4}$ denotam propriedades materiais. O tensor taxa de deformação $\mathbf{D}$ e a derivada convectada contravariante $\stackrel{\nabla}{\mathbf{D}}$ são dados por:

$$
\mathbf{D}=\nabla \mathbf{u}+(\nabla \mathbf{u})^{T}
$$

e

$$
\stackrel{\nabla}{\mathbf{D}}=\frac{D(\mathbf{D})}{D t}-\left[(\nabla \mathbf{u})^{T} \cdot \mathbf{D}+\mathbf{D} \cdot \nabla \mathbf{u}\right]
$$

respectivamente. A notação $\frac{D}{D t}$ representa a derivada material e é definida por

$$
\frac{D(\mathbf{D})}{D t}=\frac{\partial \mathbf{D}}{\partial t}+\nabla \cdot(\mathbf{u D})
$$

Para escoamentos cisalhantes simples em regime permanente, os parâmetros materiais $\lambda_{2}$ e $\lambda_{4}$ são conhecidos como o primeiro e o segundo coeficientes de diferença de tensões normais, definidos por: 


$$
\begin{aligned}
\eta & =\eta_{0}=\frac{\tau_{12}}{\mathrm{D}^{x y}} \\
\Psi_{1} & =\frac{N_{1}}{\left(\mathrm{D}^{x y}\right)^{2}}=\frac{\tau_{11}-\tau_{22}}{\left(\mathrm{D}^{x y}\right)^{2}}=-2 \eta_{0} \lambda_{2}, \\
\Psi_{2} & =\frac{N_{2}}{\left(\mathrm{D}^{x y}\right)^{2}}=\frac{\tau_{22}-\tau_{33}}{\left(\mathrm{D}^{x y}\right)^{2}}=\eta_{0} \lambda_{4},
\end{aligned}
$$

onde $N_{1}$ e $N_{2}$ são a primeira e a segunda diferenças de tensões normais, $\Psi_{1}$ e $\Psi_{2}$ são o primeiro e o segundo coeficiente de diferença de tensões normais e $\tau_{12}$ é a tensão de cisalhamento. Na notação acima, se o fluido se move somente em uma direção e sua velocidade também varia somente em uma direção, então a direção da velocidade do fluido é representada pelo índice 1, a direção de variação da velocidade é representado pelo índice 2 e a direção neutra é representado pelo índice 3 .

Na literatura (por exemplo [24], [25], [26], [27]) a equação constitutiva SOF é definida em função dos tensores de Rivlin-Ericksen $\mathbf{A}_{1}$ e $\mathbf{A}_{2}$, como apresentada a seguir,

$$
\tau=\eta_{0} \mathbf{A}_{1}+\alpha_{1} \mathbf{A}_{2}+\alpha_{2} \mathbf{A}_{1}^{2}
$$

onde $\mathbf{A}_{1}$ é igual ao tensor taxa de deformação $\mathbf{D}$, e $\mathbf{A}_{2}$ é a derivada convectada covariante, que é dada por:

$$
\mathbf{A}_{2}=\frac{D\left(\mathbf{A}_{1}\right)}{D t}+\left[(\nabla \mathbf{u})^{T} \cdot \mathbf{A}_{1}+\mathbf{A}_{1} \cdot \nabla \mathbf{u}\right]
$$

Sendo o fluido de segunda ordem modelado pela equação (1.10), para que ele seja compatível com a $2^{\mathrm{a}}$ lei da termodinâmica e também satisfaça a inequação de Clausius-Duhem para todos os movimentos e a hipótese de que a energia livre de Helmholtz específica do fluido é mínima quando está em repouso (ver [28]), então deve obedecer as restrições,

$$
\eta_{0} \geq 0, \quad \alpha_{1} \geq 0, \quad \alpha_{1}+\alpha_{2}=0
$$

Os fluidos caracterizados por essas restrições são conhecidos como fluidos de segundo grau (second-grade fluids), um caso particular dos fluidos de segunda ordem [29].

Para se ter as mesmas condições de estabilidade acima com a formulação deste trabalho, ou seja, para o modelo da equação constitutiva (1.3), apresentamos a seguir a conversão destas condições a partir do tensor taxa de deformação $\mathbf{D}=\mathbf{A}_{1}$ e da relação entre as derivadas convectadas $\stackrel{\nabla}{\mathbf{D}}=\mathbf{A}_{2}-2 \mathbf{A}_{1}^{2}$ :

$$
\begin{aligned}
\tau & =\eta_{0} \mathbf{D}+\beta_{1} \stackrel{\nabla}{\mathbf{D}}+\beta_{2} \mathbf{D}^{2} \\
& =\eta_{0} \mathbf{A}_{1}+\beta_{1}\left(\mathbf{A}_{2}-2 \mathbf{A}_{1}^{2}\right)+\beta_{2} \mathbf{A}_{1}^{2} \\
& =\eta_{0} \mathbf{A}_{1}+\beta_{1} \mathbf{A}_{2}-2 \beta_{1} \mathbf{A}_{1}^{2}+\beta_{2} \mathbf{A}_{1}^{2} \\
& =\eta_{0} \mathbf{A}_{1}+\beta_{1} \mathbf{A}_{2}+\left(\beta_{2}-2 \beta_{1}\right) \mathbf{A}_{1}^{2}
\end{aligned}
$$

como em (1.10) a condição é $\alpha_{1}+\alpha_{2}=0$, temos

$$
\beta_{1}+\left(\beta_{2}-2 \beta_{1}\right)=0
$$


$\log 0$

$$
\beta_{1}=\beta_{2} .
$$

Neste trabalho serão considerados o caso geral, onde $\beta_{1} \neq \beta_{2}$, e também o caso simplificado $\beta_{1}=\beta_{2}$. Como a taxa de variação do tensor taxa de deformação é supostamente pequena e iremos consider que os escoamentos tridimensionais convergem para uma solução estacionária, a derivada material $\frac{D(\mathbf{D})}{D t}$ será desprezada nas simulações.

Nas próximas sessões vamos considerar escoamentos cartesianos tridimensionais e apresentar as equações (1.1)-(1.6) em coordenadas cartesianas.

\subsection{Obtenção da derivada convectada contravariante em coordenadas cartesianas}

Nessa sessão, vamos expandir a derivada convectada contravariante $\stackrel{\nabla}{\mathbf{D}}$ (também chamada de derivada convectada superior) em coordenadas cartesianas tridimensionais.

O gradiente do vetor $\mathbf{u}$ e o tensor taxa de deformação $\mathbf{D}$ são dados por

$$
\begin{aligned}
& \nabla \mathbf{u}=\left[\begin{array}{lll}
\frac{\partial u}{\partial x} & \frac{\partial v}{\partial x} & \frac{\partial w}{\partial x} \\
\frac{\partial u}{\partial y} & \frac{\partial v}{\partial y} & \frac{\partial w}{\partial y} \\
\frac{\partial u}{\partial z} & \frac{\partial v}{\partial z} & \frac{\partial w}{\partial z}
\end{array}\right] \\
& \mathbf{D}=\left[\begin{array}{ccc}
2 \frac{\partial u}{\partial x} & \left(\frac{\partial u}{\partial y}+\frac{\partial v}{\partial x}\right) & \left(\frac{\partial u}{\partial z}+\frac{\partial w}{\partial x}\right) \\
\left(\frac{\partial u}{\partial y}+\frac{\partial v}{\partial x}\right) & 2 \frac{\partial v}{\partial y} & \left(\frac{\partial v}{\partial z}+\frac{\partial w}{\partial y}\right) \\
\left(\frac{\partial u}{\partial z}+\frac{\partial w}{\partial x}\right) & \left(\frac{\partial v}{\partial z}+\frac{\partial w}{\partial y}\right) & 2 \frac{\partial w}{\partial z}
\end{array}\right]
\end{aligned}
$$

onde $\mathbf{u}=(u, v, w)$ é o vetor velocidade e $\mathbf{x}=(x, y, z)$.

Para a soma da derivada temporal $\frac{\partial \mathbf{D}}{\partial t}$ e o termo convectivo $\nabla \cdot(\mathbf{u D})$ definidos na equação (1.6) temos, em notação matricial:

$$
\frac{\partial \mathbf{D}}{\partial t}+\nabla \cdot(\mathbf{u D})=\mathbf{A}=\left[\begin{array}{lll}
A^{x x} & A^{x y} & A^{x z} \\
A^{x y} & A^{y y} & A^{y z} \\
A^{x z} & A^{y z} & A^{z z}
\end{array}\right]
$$

onde seus elementos são:

$$
\begin{aligned}
& A^{x x}=\frac{\partial\left(\mathrm{D}^{x x}\right)}{\partial t}+\frac{\partial\left(u \mathrm{D}^{x x}\right)}{\partial x}+\frac{\partial\left(v \mathrm{D}^{x x}\right)}{\partial y}+\frac{\partial\left(w \mathrm{D}^{x x}\right)}{\partial z} \\
& A^{x y}=\frac{\partial\left(\mathrm{D}^{x y}\right)}{\partial t}+\frac{\partial\left(u \mathrm{D}^{x y}\right)}{\partial x}+\frac{\partial\left(v \mathrm{D}^{x y}\right)}{\partial y}+\frac{\partial\left(w \mathrm{D}^{x y}\right)}{\partial z} \\
& A^{x z}=\frac{\partial\left(\mathrm{D}^{x z}\right)}{\partial t}+\frac{\partial\left(u \mathrm{D}^{x z}\right)}{\partial x}+\frac{\partial\left(v \mathrm{D}^{x z}\right)}{\partial y}+\frac{\partial\left(w \mathrm{D}^{x z}\right)}{\partial z} \\
& A^{y y}=\frac{\partial\left(\mathrm{D}^{y y}\right)}{\partial t}+\frac{\partial\left(u \mathrm{D}^{y y}\right)}{\partial x}+\frac{\partial\left(v \mathrm{D}^{y y}\right)}{\partial y}+\frac{\partial\left(w \mathrm{D}^{y y}\right)}{\partial z} \\
& A^{y z}=\frac{\partial\left(\mathrm{D}^{y z}\right)}{\partial t}+\frac{\partial\left(u \mathrm{D}^{y z}\right)}{\partial x}+\frac{\partial\left(v \mathrm{D}^{y z}\right)}{\partial y}+\frac{\partial\left(w \mathrm{D}^{y z}\right)}{\partial z} \\
& A^{z z}=\frac{\partial\left(\mathrm{D}^{z z}\right)}{\partial t}+\frac{\partial\left(u \mathrm{D}^{z z}\right)}{\partial x}+\frac{\partial\left(v \mathrm{D}^{z z}\right)}{\partial y}+\frac{\partial\left(w \mathrm{D}^{z z}\right)}{\partial z}
\end{aligned}
$$


sendo as componentes $\mathrm{D}^{x x}, \mathrm{D}^{x y}, \mathrm{D}^{x z}, \mathrm{D}^{z y}, \mathrm{D}^{y z}$ e $\mathrm{D}^{z z}$ do tensor taxa de deformação obtidas pela equação (1.13).

Utilizando as equações (1.12) e (1.13) obtemos:

$$
(\nabla \mathbf{u})^{T} \mathbf{D}=\mathbf{B}=\left[\begin{array}{lll}
B^{x x} & B^{x y} & B^{x z} \\
B^{y x} & B^{y y} & B^{y z} \\
B^{z x} & B^{z y} & B^{z z}
\end{array}\right]
$$

onde:

$$
\begin{aligned}
B^{x x} & =\frac{\partial u}{\partial x} \mathrm{D}^{x x}+\frac{\partial u}{\partial y} \mathrm{D}^{x y}+\frac{\partial u}{\partial z} \mathrm{D}^{x z} \\
B^{x y} & =\frac{\partial u}{\partial x} \mathrm{D}^{x y}+\frac{\partial u}{\partial y} \mathrm{D}^{y y}+\frac{\partial u}{\partial z} \mathrm{D}^{y z} \\
B^{x z} & =\frac{\partial u}{\partial x} \mathrm{D}^{x z}+\frac{\partial u}{\partial y} \mathrm{D}^{y z}+\frac{\partial u}{\partial z} \mathrm{D}^{z z} \\
B^{y x} & =\frac{\partial v}{\partial x} \mathrm{D}^{x x}+\frac{\partial v}{\partial y} \mathrm{D}^{x y}+\frac{\partial v}{\partial z} \mathrm{D}^{x z} \\
B^{y y} & =\frac{\partial v}{\partial x} \mathrm{D}^{x y}+\frac{\partial v}{\partial y} \mathrm{D}^{y y}+\frac{\partial v}{\partial z} \mathrm{D}^{y z} \\
B^{y z} & =\frac{\partial v}{\partial x} \mathrm{D}^{x z}+\frac{\partial v}{\partial y} \mathrm{D}^{y z}+\frac{\partial v}{\partial z} \mathrm{D}^{z z} \\
B^{z x} & =\frac{\partial w}{\partial x} \mathrm{D}^{x x}+\frac{\partial w}{\partial y} \mathrm{D}^{x y}+\frac{\partial w}{\partial z} \mathrm{D}^{x z} \\
B^{z y} & =\frac{\partial w}{\partial x} \mathrm{D}^{x y}+\frac{\partial w}{\partial y} \mathrm{D}^{y y}+\frac{\partial w}{\partial z} \mathrm{D}^{y z} \\
B^{z z} & =\frac{\partial w}{\partial x} \mathrm{D}^{x z}+\frac{\partial w}{\partial y} \mathrm{D}^{y z}+\frac{\partial w}{\partial z} \mathrm{D}^{z z}
\end{aligned}
$$

De maneira análoga, obtém-se

$$
\mathbf{D} \nabla \mathbf{u}=\mathbf{C}=\left[\begin{array}{lll}
C^{x x} & C^{x y} & C^{x z} \\
C^{y x} & C^{y y} & C^{y z} \\
C^{z x} & C^{z y} & C^{z z}
\end{array}\right]
$$


onde:

$$
\begin{aligned}
C^{x x} & =\frac{\partial u}{\partial x} \mathrm{D}^{x x}+\frac{\partial u}{\partial y} \mathrm{D}^{x y}+\frac{\partial u}{\partial z} \mathrm{D}^{x z} \\
C^{x y} & =\frac{\partial v}{\partial x} \mathrm{D}^{x x}+\frac{\partial v}{\partial y} \mathrm{D}^{x y}+\frac{\partial v}{\partial z} \mathrm{D}^{x z} \\
C^{x z} & =\frac{\partial w}{\partial x} \mathrm{D}^{x x}+\frac{\partial w}{\partial y} \mathrm{D}^{x y}+\frac{\partial w}{\partial z} \mathrm{D}^{x z} \\
C^{y x} & =\frac{\partial u}{\partial x} \mathrm{D}^{x y}+\frac{\partial u}{\partial y} \mathrm{D}^{y y}+\frac{\partial u}{\partial z} \mathrm{D}^{y z} \\
C^{y y} & =\frac{\partial v}{\partial x} \mathrm{D}^{x y}+\frac{\partial v}{\partial y} \mathrm{D}^{y y}+\frac{\partial v}{\partial z} \mathrm{D}^{y z} \\
C^{y z} & =\frac{\partial w}{\partial x} \mathrm{D}^{x y}+\frac{\partial w}{\partial y} \mathrm{D}^{y y}+\frac{\partial w}{\partial z} \mathrm{D}^{y z} \\
C^{z x} & =\frac{\partial u}{\partial x} \mathrm{D}^{x z}+\frac{\partial u}{\partial y} \mathrm{D}^{y z}+\frac{\partial u}{\partial z} \mathrm{D}^{z z} \\
C^{z y} & =\frac{\partial v}{\partial x} \mathrm{D}^{x z}+\frac{\partial v}{\partial y} \mathrm{D}^{y z}+\frac{\partial v}{\partial z} \mathrm{D}^{z z} \\
C^{z z} & =\frac{\partial w}{\partial x} \mathrm{D}^{x z}+\frac{\partial w}{\partial y} \mathrm{D}^{y z}+\frac{\partial w}{\partial z} \mathrm{D}^{z z}
\end{aligned}
$$

Finalmente, utilizando a notação para as componentes da derivada convectada contravariante $\stackrel{\nabla}{\mathrm{D}}$ :

$$
\overrightarrow{\mathbf{D}}=\left[\begin{array}{ccc}
\nabla & \nabla & \nabla \\
\mathrm{D}^{x x} & \mathrm{D}^{x y} & \mathrm{D}^{x z} \\
\nabla & \nabla & \nabla \\
\mathrm{D}^{x y} & \mathrm{D}^{y y} & \mathrm{D}^{y z} \\
\nabla & \nabla & \nabla \\
\mathrm{D}^{x z} & \mathrm{D}^{y z} & \mathrm{D}^{z z}
\end{array}\right]
$$

e das equações $(1.14),(1.21)$ e $(1.31)$, temos que as componentes do tensor $\stackrel{\nabla}{\mathbf{D}}$ são dadas por:

$$
\stackrel{\nabla}{\mathbf{D}}=\mathbf{A}-[\mathbf{B}+\mathbf{C}]
$$

Logo,

$$
\begin{aligned}
& \stackrel{\nabla}{\mathrm{D}^{x x}}=A^{x x}-\left[B^{x x}+C^{x x}\right] \\
& \stackrel{\nabla}{\mathrm{D}^{x y}}=A^{x y}-\left[B^{x y}+C^{x y}\right] \\
& \stackrel{\nabla}{\mathrm{D}^{x z}}=A^{x z}-\left[B^{x z}+C^{x z}\right] \\
& \stackrel{\nabla}{\mathrm{D}^{y y}}=A^{y y}-\left[B^{y y}+C^{y y}\right] \\
& \stackrel{\nabla}{\mathrm{D}^{y z}}=A^{y z}-\left[B^{y z}+C^{y z}\right] \\
& \stackrel{\nabla}{\mathrm{D}^{z z}}=A^{z z}-\left[B^{z z}+C^{z z}\right]
\end{aligned}
$$




\subsection{Obtenção das equações governantes em coordenadas cartesianas}

Utilizando a equação (1.13), pode-se verificar que o produto (tensorial) $\mathbf{D} \cdot \mathbf{D}$ é dado por:

$$
\mathbf{D} \cdot \mathbf{D}=\mathcal{D}=\left[\begin{array}{lll}
\mathcal{D}^{x x} & \mathcal{D}^{x y} & \mathcal{D}^{x z} \\
\mathcal{D}^{x y} & \mathcal{D}^{y y} & \mathcal{D}^{y z} \\
\mathcal{D}^{x z} & \mathcal{D}^{y z} & \mathcal{D}^{z z}
\end{array}\right]
$$

onde

$$
\begin{aligned}
& \mathcal{D}^{x x}=\left(\mathrm{D}^{x x}\right)^{2}+\left(\mathrm{D}^{x y}\right)^{2}+\left(\mathrm{D}^{x z}\right)^{2} \\
& \mathcal{D}^{x y}=\mathrm{D}^{x x} \mathrm{D}^{x y}+\mathrm{D}^{x y} \mathrm{D}^{y y}+\mathrm{D}^{x z} \mathrm{D}^{y z} \\
& \mathcal{D}^{x z}=\mathrm{D}^{x x} \mathrm{D}^{x z}+\mathrm{D}^{x y} \mathrm{D}^{y z}+\mathrm{D}^{x z} \mathrm{D}^{z z} \\
& \mathcal{D}^{y y}=\left(\mathrm{D}^{x y}\right)^{2}+\left(\mathrm{D}^{y y}\right)^{2}+\left(\mathrm{D}^{y z}\right)^{2} \\
& \mathcal{D}^{y z}=\mathrm{D}^{x y} \mathrm{D}^{x z}+\mathrm{D}^{y y} \mathrm{D}^{y z}+\mathrm{D}^{y z} \mathrm{D}^{z z} \\
& \mathcal{D}^{z z}=\left(\mathrm{D}^{x z}\right)^{2}+\left(\mathrm{D}^{y z}\right)^{2}+\left(\mathrm{D}^{z z}\right)^{2}
\end{aligned}
$$

Logo, podemos escrever as componentes da equação constitutiva (1.3) na forma:

$$
\begin{aligned}
& \tau^{x x}=\eta_{0}\left[\mathrm{D}^{x x}+\lambda_{2} \mathrm{D}^{x x}+\lambda_{4} \mathcal{D}^{x x}\right] \\
& \tau^{x y}=\eta_{0}\left[\mathrm{D}^{x y}+\lambda_{2} \mathrm{D}^{x y}+\lambda_{4} \mathcal{D}^{x y}\right] \\
& \tau^{x z}=\eta_{0}\left[\mathrm{D}^{x z}+\lambda_{2} \mathrm{D}^{x z}+\lambda_{4} \mathcal{D}^{x z}\right] \\
& \tau^{y y}=\eta_{0}\left[\mathrm{D}^{y y}+\lambda_{2} \mathrm{D}^{y y}+\lambda_{4} \mathcal{D}^{y y}\right] \\
& \tau^{y z}=\eta_{0}\left[\mathrm{D}^{y z}+\lambda_{2} \mathrm{D}^{y z}+\lambda_{4} \mathcal{D}^{y z}\right] \\
& \tau^{z z}=\eta_{0}\left[\mathrm{D}^{z z}+\lambda_{2} \mathrm{D}^{z z}+\lambda_{4} \mathcal{D}^{z z}\right]
\end{aligned}
$$

onde $\mathcal{D}^{x x}, \mathcal{D}^{x y}, \ldots, \mathcal{D}^{z z}$ são obtidos por meio das equações (1.50)-(1.55), respectivamente e $\stackrel{\nabla}{D^{x x}}, \stackrel{\nabla}{\mathrm{D}^{x y}}, \ldots, \stackrel{\nabla}{\mathrm{D}^{z z}}$ são calculados pelas equações (1.43)-(1.48), respectivamente.

Para facilitar a notação, vamos escrever o tensor de tensão extra como uma soma de um tensor newtoniano, representado por $\eta_{0} \mathbf{D}$, e um tensor não-newtoniano que representaremos por $\eta_{0} \boldsymbol{\Phi}$, onde $\boldsymbol{\Phi}$ é a função reológica descrita por

$$
\boldsymbol{\Phi}=\lambda_{2} \stackrel{\nabla}{\mathbf{D}}+\lambda_{4}(\mathbf{D} \cdot \mathbf{D})
$$

dessa forma temos:

$$
\boldsymbol{\tau}=\eta_{0}[\mathbf{D}+\boldsymbol{\Phi}]
$$

logo, as equações (1.56)-(1.61) podem ser escritas como: 
componente $\tau^{x x}$ :

$$
\tau^{x x}=\eta_{0}\left[\mathrm{D}^{x x}+\Phi^{x x}\right]
$$

onde a componente não-newtoniana $\Phi^{x x}$ é dada por:

$$
\Phi^{x x}=\lambda_{2}\left\{A^{x x}-\left[B^{x x}+C^{x x}\right]\right\}+\lambda_{4}\left[\mathcal{D}^{x x}\right]
$$

sendo $A^{x x}, B^{x x}, C^{x x}, \mathcal{D}^{x x}$ dados por (1.15), (1.22), (1.32) e (1.50), respectivamente.

componente $\tau^{x y}$ :

$$
\tau^{x y}=\eta_{0}\left[\mathrm{D}^{x y}+\Phi^{x y}\right]
$$

onde a componente não-newtoniana $\Phi^{x y}$ é dada por:

$$
\Phi^{x y}=\lambda_{2}\left\{A^{x y}-\left[B^{x y}+C^{x y}\right]\right\}+\lambda_{4}\left[\mathcal{D}^{x y}\right]
$$

componente $\tau^{x z}$ :

$$
\tau^{x z}=\eta_{0}\left[\mathrm{D}^{x z}+\Phi^{x z}\right]
$$

onde a componente não-newtoniana $\Phi^{x z}$ é dada por:

$$
\Phi^{x z}=\lambda_{2}\left\{A^{x z}-\left[B^{x z}+C^{x z}\right]\right\}+\lambda_{4}\left[\mathcal{D}^{x z}\right]
$$

componente $\tau^{y y}$ :

$$
\tau^{y y}=\eta_{0}\left[\mathrm{D}^{y y}+\Phi^{y y}\right],
$$

onde a componente não-newtoniana $\Phi^{y y}$ é dada por:

$$
\Phi^{y y}=\lambda_{2}\left\{A^{y y}-\left[B^{y y}+C^{y y}\right]\right\}+\lambda_{4}\left[\mathcal{D}^{y y}\right]
$$

componente $\tau^{y z}$ :

$$
\tau^{y z}=\eta_{0}\left[\mathrm{D}^{y z}+\Phi^{y z}\right]
$$

onde a componente não-newtoniana $\Phi^{y z}$ é dada por:

$$
\Phi^{y z}=\lambda_{2}\left\{A^{y z}-\left[B^{y z}+C^{y z}\right]\right\}+\lambda_{4}\left[\mathcal{D}^{y z}\right]
$$

componente $\tau^{z z}$ :

$$
\tau^{z z}=\eta_{0}\left[\mathrm{D}^{z z}+\Phi^{z z}\right]
$$

onde a componente não-newtoniana $\Phi^{z z}$ é dada por:

$$
\Phi^{z z}=\lambda_{2}\left\{A^{z z}-\left[B^{z z}+C^{z z}\right]\right\}+\lambda_{4}\left[\mathcal{D}^{z z}\right]
$$

Tendo obtido a equação constitutiva do modelo SOF expandida em coordenadas cartesianas tridimensionais conforme a equação (1.63), a equação de conservação de quantidade de movimento (1.1) toma a forma

$$
\frac{\partial \mathbf{u}}{\partial t}+\nabla \cdot(\mathbf{u u})=-\nabla p^{*}+\left(\frac{\eta_{0}}{\rho}\right)\left[\nabla^{2} \mathbf{u}+\nabla \cdot \mathbf{\Phi}\right]+\mathbf{g} .
$$


onde $p^{*}=(p / \rho)$ denota a pressão por unidade de massa específica. Por simplicificação, $p^{*}$ será representado apenas por $p$. Em coordenas cartesianas, as componentes $u, v, w$ são:

$$
\begin{aligned}
\frac{\partial u}{\partial t}+ & \frac{\partial\left(u^{2}\right)}{\partial x}+\frac{\partial(u v)}{\partial y}+\frac{\partial(u w)}{\partial z}=-\frac{\partial p}{\partial x}+ \\
& +\frac{\eta_{0}}{\rho}\left[\nabla^{2} u+\frac{\partial \Phi^{x x}}{\partial x}+\frac{\partial \Phi^{x y}}{\partial y}+\frac{\partial \Phi^{x z}}{\partial z}\right]+g_{x}, \\
\frac{\partial v}{\partial t}+ & \frac{\partial(u v)}{\partial x}+\frac{\partial\left(v^{2}\right)}{\partial y}+\frac{\partial(v w)}{\partial z}=-\frac{\partial p}{\partial y}+ \\
& +\frac{\eta_{0}}{\rho}\left[\nabla^{2} v+\frac{\partial \Phi^{x y}}{\partial x}+\frac{\partial \Phi^{y y}}{\partial y}+\frac{\partial \Phi^{y z}}{\partial z}\right]+g_{y}, \\
\frac{\partial w}{\partial t}+ & \frac{\partial(u w)}{\partial x}+\frac{\partial(v w)}{\partial y}+\frac{\partial\left(w^{2}\right)}{\partial z}=-\frac{\partial p}{\partial z}+ \\
& +\frac{\eta_{0}}{\rho}\left[\nabla^{2} w+\frac{\partial \Phi^{x z}}{\partial x}+\frac{\partial \Phi^{y z}}{\partial y}+\frac{\partial \Phi^{z z}}{\partial z}\right]+g_{z},
\end{aligned}
$$

onde $\Phi^{x x}, \Phi^{x y}, \ldots, \Phi^{z z}$ são dadas por (1.64), (1.65), . ., (1.69), respectivamente.

A equação da continuidade (1.2) é dada por:

$$
\frac{\partial u}{\partial x}+\frac{\partial v}{\partial y}+\frac{\partial w}{\partial z}=0
$$

Com a expansão das equações governantes, finalizamos o desenvolvimento do modelo SOF incompressível no sistema de coordenadas adotado. O próximo passo é adimensionar as equações governantes.

\subsection{Adimensionalização das equações governantes}

Para adimensionar as equações governantes, vamos utilizar $U$ e $L$ valores de referência para a velocidade e para o comprimento, $g$ um valor de referência para a constante gravitacional e as variáveis adimensionais:

$$
\overline{\mathbf{x}}=\frac{\mathbf{x}}{L}, \quad \overline{\mathbf{u}}=\frac{\mathbf{u}}{U}, \quad \bar{p}=\frac{p}{\rho U^{2}}, \quad \overline{\mathbf{g}}=\frac{\mathbf{g}}{g}, \quad \bar{t}=\frac{U}{L} t .
$$

Nesse caso, pode ser mostrado que a equação de conservação de massa, a equação de conservação de quantidade de movimento (1.70) e a equação constitutiva (1.63) podem ser escritas na forma adimensional como (para detalhes, ver Tomé et al. [13]):

$$
\begin{aligned}
\nabla \cdot \mathbf{u} & =0 \\
\frac{\partial \mathbf{u}}{\partial t} & =-\nabla \cdot(\mathbf{u u})-\nabla p+\left(\frac{1}{R e}\right)\left[\nabla^{2} \mathbf{u}+\nabla \cdot \boldsymbol{\Phi}\right]+\frac{1}{F r^{2}} \mathbf{g}
\end{aligned}
$$

onde

$$
\mathbf{\Phi}=D e[\mathbf{A}-(\mathbf{B}+\mathbf{C})]+\kappa \mathcal{D}
$$

e A, B, C e $\mathcal{D}$ são obtidos pelas equações (1.14), (1.21), (1.31) e (1.49), respectivamente. As barras foram omitidas para simplificação.

Nas equacões acima, $R e=\frac{\rho U L}{\eta_{0}}$ é o número de Reynolds, $F r=\frac{U}{\sqrt{g L}}$ é o número de Froude, $D e=\frac{\lambda_{2} U}{L}$ é o número de Deborah e o número adimensional $\kappa$ é definido como $\kappa=\frac{\lambda_{4} U}{L}$. 
As equações (1.75)-(1.77) são as equações governantes na forma adimensional para simular escoamentos incompressíveis de fluidos de segunda ordem (para simplificar a notação, o sinal de barra em cima das variáveis foi omitido). A partir desse capítulo, trabalharemos somente com as equações adimensionais. A seguir, trataremos as condições de contorno necessárias para escoamento do tipo SOF.

\subsection{Condições de contorno nos injetores, ejetores e con- tornos rígidos}

Os contornos da malha podem ser de vários tipos: injetores, ejetores ou parede rígida. Sejam $u_{n}, u_{m 1}, u_{m 2}$ a velocidade normal e tangentes ao contorno, respectivamente. Então, para um contorno tipo parede rígida, temos

$$
u_{n}=0 \quad u_{m 1}=0 \quad u_{m 2}=0 \quad \text { (condição não-escorregamento) }
$$

e em injetores a velocidade normal é prescrita e as velocidades tangenciais são nulas, ou seja

$$
u_{n}=U_{i n j} \quad u_{m 1}=0 \quad u_{m 2}=0 .
$$

Em ejetores, assumimos a condiçao de Neumann homogênea,

$$
\frac{\partial u_{n}}{\partial n}=\frac{\partial u_{m 1}}{\partial n}=\frac{\partial u_{m 2}}{\partial n}=0
$$

\subsection{Condições de contorno nas superfícies livres}

Considera-se um fluido viscoso escoando em uma atmosfera passiva (onde assume-se pressão zero), de forma que as componentes normal e tangencial da tensão devem ser contínuas através da superfície livre. Assume-se também que os efeitos da tensão superficial podem ser desprezados (para detalhes ver Batchelor [1]). Nesse caso, as condições a serem satisfeitas são:

$$
\begin{array}{r}
\mathbf{n} \cdot(\boldsymbol{\sigma} \cdot \mathbf{n})=\mathbf{0} \\
\mathbf{m} \mathbf{1} \cdot(\boldsymbol{\sigma} \cdot \mathbf{n})=\mathbf{0} \\
\mathbf{m} \mathbf{2} \cdot(\boldsymbol{\sigma} \cdot \mathbf{n})=\mathbf{0}
\end{array}
$$

onde $\boldsymbol{\sigma}$ é o tensor tensão total dado por

$$
\boldsymbol{\sigma}=-p \mathbf{I}+\frac{1}{R e}[\mathbf{D}+\mathbf{\Phi}]
$$

onde $\mathbf{n}=\left(n_{x}, n_{y}, n_{z}\right)$ é o vetor unitário normal à superfície livre e $\mathbf{m} \mathbf{1}, \mathbf{m} \mathbf{2}$ são os vetores unitários tangenciais. Em coordenadas cartesianas estas equações são dadas por:

$$
\begin{aligned}
\tilde{p}= & \frac{1}{R e}\left[2 \frac{\partial u}{\partial x} n_{x}^{2}+2 \frac{\partial v}{\partial y} n_{y}^{2}+2 \frac{\partial w}{\partial z} n_{z}^{2}+2\left(\frac{\partial u}{\partial y}+\frac{\partial v}{\partial x}\right) n_{x} n_{y}\right. \\
& +2\left(\frac{\partial u}{\partial z}+\frac{\partial w}{\partial x}\right) n_{x} n_{z}+2\left(\frac{\partial v}{\partial z}+\frac{\partial w}{\partial y}\right) n_{y} n_{z}+\Phi^{x x} n_{x}^{2}+\Phi^{y y} n_{y}^{2} \\
& \left.+\Phi^{z z} n_{z}^{2}+2\left(\Phi^{x y} n_{x} n_{y}+\Phi^{x z} n_{x} n_{z}+\Phi^{y z} n_{y} n_{z}\right)\right]
\end{aligned}
$$




$$
\begin{aligned}
& 2 \frac{\partial u}{\partial x} n_{x} m 1_{x}+2 \frac{\partial v}{\partial y} n_{y} m 1_{y}+2 \frac{\partial w}{\partial z} n_{z} m 1_{z} \\
& +\left(\frac{\partial u}{\partial y}+\frac{\partial v}{\partial x}\right)\left(n_{x} m 1_{y}+n_{y} m 1_{x}\right)+\left(\frac{\partial u}{\partial z}+\frac{\partial w}{\partial x}\right)\left(n_{x} m 1_{z}+n_{z} m 1_{x}\right) \\
& +\left(\frac{\partial v}{\partial z}+\frac{\partial w}{\partial y}\right)\left(n_{y} m 1_{z}+n_{z} m 1_{y}\right)=-\left[\Phi^{x x} n_{x} m 1_{x}+\Phi^{y y} n_{y} m 1_{y}+\Phi^{z z} n_{z} m 1_{z}\right. \\
& \left.+\Phi^{x y}\left(n_{x} m 1_{y}+n_{y} m 1_{x}\right)+\Phi^{x z}\left(n_{x} m 1_{z}+n_{z} m 1_{x}\right)+\Phi^{y z}\left(n_{y} m 1_{z}+n_{z} m 1_{y}\right)\right]
\end{aligned}
$$

$\mathrm{e}$

$$
\begin{aligned}
& 2 \frac{\partial u}{\partial x} n_{x} m 2_{x}+2 \frac{\partial v}{\partial y} n_{y} m 2_{y}+2 \frac{\partial w}{\partial z} n_{z} m 2_{z} \\
& +\left(\frac{\partial u}{\partial y}+\frac{\partial v}{\partial x}\right)\left(n_{x} m 2_{y}+n_{y} m 2_{x}\right)+\left(\frac{\partial u}{\partial z}+\frac{\partial w}{\partial x}\right)\left(n_{x} m 2_{z}+n_{z} m 2_{x}\right) \\
& +\left(\frac{\partial v}{\partial z}+\frac{\partial w}{\partial y}\right)\left(n_{y} m 2_{z}+n_{z} m 2_{y}\right)=-\left[\Phi^{x x} n_{x} m 2_{x}+\Phi^{y y} n_{y} m 2_{y}+\Phi^{z z} n_{z} m 2_{z}\right. \\
& \left.+\Phi^{x y}\left(n_{x} m 2_{y}+n_{y} m 2_{x}\right)+\Phi^{x z}\left(n_{x} m 2_{z}+n_{z} m 2_{x}\right)+\Phi^{y z}\left(n_{y} m 2_{z}+n_{z} m 2_{y}\right)\right]
\end{aligned}
$$

As equações (1.82)-(1.84) representam as condições de contorno apropriadas para a superfície livre do fluido. A aproximação por diferenças finitas destas equações serão dadas no capítulo 3. 


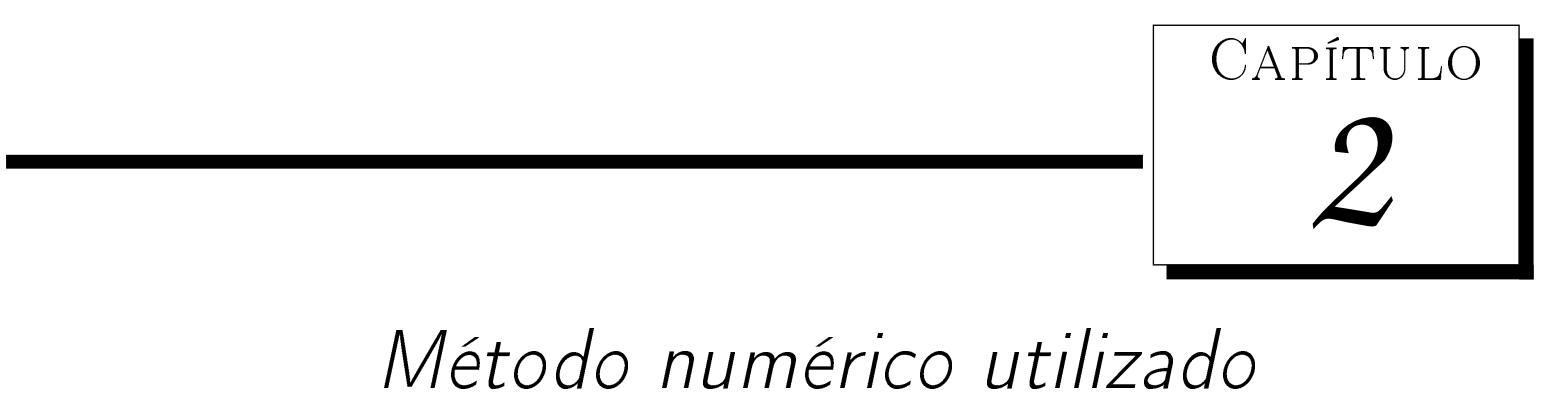

\subsection{GENSMAC3D-SOF}

Para resolver as equações da continuidade (1.75), conservação de quantidade de movimento (1.76) e a equação reológica (1.77) vamos nos basear na metodologia apresentada por Tomé et al. [13] para os escoamentos bidimensionais de fluidos de segunda ordem. Neste trabalho vamos apresentar a extensão dessa metodologia para escoamentos tridimensionais como segue.

Assume-se que, no tempo $t_{n}$, a velocidade é conhecida, bem como as condições de contorno para $\mathbf{u}\left(\mathbf{x}, t_{n}\right)$. Seja $t_{n+1}=t_{n}+\delta t$, então, a velocidade $\mathbf{u}\left(\mathbf{x}, t_{n+1}\right)$ e a pressão $p\left(\mathbf{x}, t_{n+1}\right)$ são calculadas pelos seguintes passos:

\subsection{Algoritmo computacional}

Passo 1: Com $\mathbf{u}\left(\mathbf{x}, t_{n}\right)$, calcule as componentes de $\boldsymbol{\Phi}\left(\mathbf{x}, t_{n}\right)$ usando equações (1.64)-(1.69).

Passo 2: Seja $\tilde{p}\left(\mathbf{x}, t_{n}\right)$ um campo de pressão inicial que satisfaz a condição de contorno para a pressão na superfície livre. Esse campo de pressão é calculado de modo que a equação (1.82) seja satisfeita na superfície livre.

Passo 3: Com a função reológica $\boldsymbol{\Phi}\left(\mathbf{x}, t_{n}\right)$ e a pressão $\tilde{p}\left(\mathbf{x}, t_{n}\right)$, calcula-se a velocidade intermediária $\widetilde{\mathbf{u}}\left(\mathbf{x}, t_{n+1}\right)$ por meio da equação

$$
\frac{\partial \widetilde{\mathbf{u}}}{\partial t}=-\nabla \cdot(\mathbf{u u})-\nabla \tilde{p}+\left(\frac{1}{R e}\right)\left[\nabla^{2} \mathbf{u}+\nabla \cdot \boldsymbol{\Phi}\right]+\frac{1}{F r^{2}} \mathbf{g}
$$

com $\widetilde{\mathbf{u}}\left(\mathbf{x}, t_{n}\right)=\mathbf{u}\left(\mathbf{x}, t_{n}\right)$ inclusive para as condições de contorno. Subtraindo-se (2.1) de (1.76), temos

$$
\frac{\partial(\mathbf{u}-\widetilde{\mathbf{u}})}{\partial t}=-\nabla(p-\tilde{p})
$$


Aplicando-se o operador rotacional em (2.2), obtém-se

$$
\nabla \times\left(\frac{\partial(\mathbf{u}-\widetilde{\mathbf{u}})}{\partial t}\right)=-\nabla \times(\nabla(p-\tilde{p}))=0 .
$$

Como é válida a comutação entre os operadores em (2.3), tem-se

$$
\frac{\partial(\nabla \times(\mathbf{u}-\widetilde{\mathbf{u}}))}{\partial t}=0
$$

ou seja, $\nabla \times(\mathbf{u}-\widetilde{\mathbf{u}})=\mathbf{f}(\mathbf{x}), t \in\left[t_{n}, t_{n+1}\right]$. Porém, como $\widetilde{\mathbf{u}}\left(\mathbf{x}, t_{n}\right)=\mathbf{u}\left(\mathbf{x}, t_{n}\right)$, conclui-se que $\mathbf{f}(\mathbf{x})=0$ e portanto

$$
\nabla \times \widetilde{\mathbf{u}}\left(\mathbf{x}, t_{n+1}\right)=\nabla \times \mathbf{u}\left(\mathbf{x}, t_{n+1}\right),
$$

o que mostra que $\widetilde{\mathbf{u}}\left(\mathbf{x}, t_{n+1}\right)$ contém a vorticidade correta em $t=t_{n+1}$. Porém, seu cálculo através de (2.1) não garante que a equação da continuidade seja satisfeita.

Seja $\psi\left(\mathbf{x}, t_{n+1}\right)$ uma função escalar que satisfaça a equação de Poisson

$$
\nabla^{2} \psi\left(\mathbf{x}, t_{n+1}\right)=\nabla \cdot \widetilde{\mathbf{u}}\left(\mathbf{x}, t_{n+1}\right)
$$

Define-se $\mathbf{u}\left(\mathbf{x}, t_{n+1}\right)$ por

$$
\mathbf{u}\left(\mathbf{x}, t_{n+1}\right)=\widetilde{\mathbf{u}}\left(\mathbf{x}, t_{n+1}\right)-\nabla \psi\left(\mathbf{x}, t_{n+1}\right)
$$

Logo, $\mathbf{u}\left(\mathbf{x}, t_{n+1}\right)$ contém a mesma vorticidade (gerada por $\widetilde{\mathbf{u}}\left(\mathbf{x}, t_{n}\right)$ ) e adicionalmente, conserva a massa.

Passo 4: Resolver a equação de Poisson (2.6) com condições de contorno $\psi\left(\mathbf{x}, t_{n+1}\right)=0$ na superfície livre e ejetores, e com $\frac{\partial \psi\left(\mathbf{x}, t_{n+1}\right)}{\partial n}=0$ em contorno rígidos e injetores.

Passo 5: Calcular a velocidade final $\mathbf{u}\left(\mathbf{x}, t_{n+1}\right)$ através da equação $(2.7)$.

Passo 6: Utilizando as equações (2.2) e (2.7), obtém-se, para o cálculo da pressão final, a seguinte equação:

$$
p\left(\mathbf{x}, t_{n+1}\right)=\tilde{p}\left(\mathbf{x}, t_{n}\right)+\frac{\psi\left(\mathbf{x}, t_{n+1}\right)}{\delta t} .
$$

Passo 7: O último passo é atualizar a posição das partículas marcadoras que descrevem a superfície do fluido resolvendo as equações

$$
\frac{\partial \mathbf{x}}{\partial t}=\mathbf{u}\left(\mathbf{x}, t_{n+1}\right)
$$

pelo método de Euler explícito. A superfície do fluido é uma superfície em três dimensões que é composta por quadriláteros e triângulos cujos vértices são definidos pelas partículas marcadoras. Neste passo, após a solução da equação (2.9) essas partículas são movidas para suas novas posições e a superfície do fluido é atualizada. 


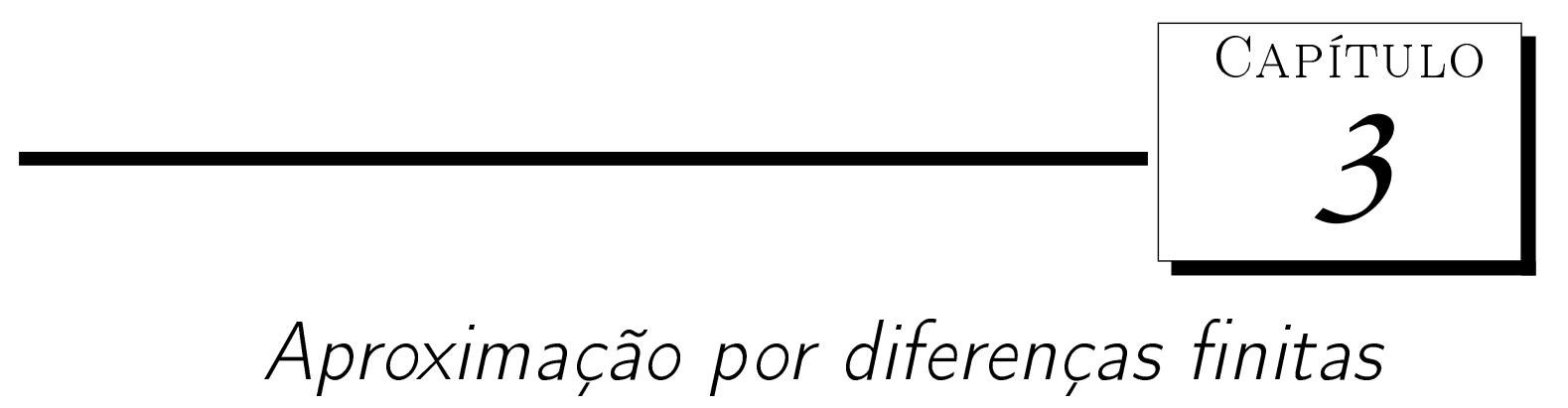

Para resolver as equações do algoritmo computacional vamos utilizar o método de diferenças finitas e uma malha deslocada ("staggered grid"). Nessa malha, as componentes da velocidade $(u, v, w)$ são posicionadas nas faces da célula, enquanto que a pressão $(p) \mathrm{e}$ a função reológica $(\boldsymbol{\Phi})$ são calculadas no centro das células. A Figura (3.1-a) ilustra um exemplo típico das células da malha. Pretendemos simular problemas transientes onde o fluido (também a superfície livre) está em movimento. Assim, um esquema para identificar a região do fluido bem como a localização da superfície livre é empregado. Para isso, as células da malha são classificadas como:

- Vazia (E) - células que não contêm fluido.

- Cheia (F) - células que possuem fluido e não estão em contato com faces de células vazias.

- Superfície (S) - células que representam a superfície livre. Essas células contêm fluido e possuem pelo menos uma face em contato com faces de células vazias.

- Contorno (B) - Célula que delimita uma fronteira rígida. Nessa célula é aplicada a condição não-escorregamento.

- Injetoras (I) - células que definem um injetor ("inflow").

- Ejetoras $(\mathrm{O})$ - células que definem um ejetor ("outflow").

A Figura (3.1-b) ilustra os tipos de células no domínio computacional.

\subsection{Discretização da equação de conservação de quan- tidade de movimento}

As componentes da velocidade intermediária $\widetilde{u}\left(\mathbf{x}, t_{n+1}\right), \widetilde{v}\left(\mathbf{x}, t_{n+1}\right)$ e $\widetilde{w}\left(\mathbf{x}, t_{n+1}\right)$ são calculadas por meio da equação (2.1). Essas equações são aproximadas por diferenças finitas e são aplicadas nas faces $\left(i+\frac{1}{2}, j, k\right),\left(i, j+\frac{1}{2}, k\right)$ e $\left(i, j, k+\frac{1}{2}\right)$, respectivamente. Os termos lineares são aproximados por diferenças de segunda ordem, enquanto que os termos não-lineares são 
a)

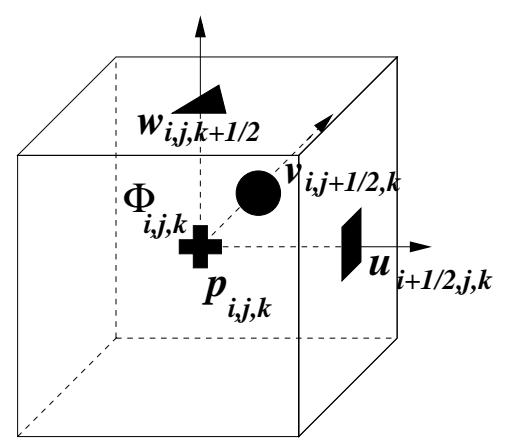

b)

\begin{tabular}{|c|c|c|c|c|c|c|c|c|c|c|c|c|c|c|c|c|c|c|c|c|}
\hline & B & B & B & B & B & B & B & B & B & B & B & B & B & B & B & B & B & B & B & \\
\hline I & $F$ & $\mathrm{~F}$ & $\mathrm{~F}$ & $F$ & $\mathrm{~F}$ & 5 & $S$ & & & & & & & & & & & & & 0 \\
\hline 1 & $\mathrm{~F}$ & $\mathrm{~F}$ & $\mathrm{~F}$ & $F$ & $\mathrm{~F}$ & $\mathrm{~F}$ & s) & & & & & & & & & & & & & 0 \\
\hline I & $\mathrm{F}$ & $\mathrm{F}$ & $\mathrm{F}$ & $\mathrm{F}$ & $\mathrm{F}$ & $\mathrm{F}$ & $\mathrm{S}$ & & & & & $\delta x$ & & & & & & & & 0 \\
\hline I & $\mathrm{F}$ & $F$ & $\mathrm{~F}$ & $F$ & $\mathrm{~F}$ & $\mathrm{~F}$ & $\mathrm{~s}$ & & & & & E & $1 \delta \mathrm{y}$ & & & & & & & 0 \\
\hline I & $\mathrm{F}$ & $\mathrm{F}$ & $\mathrm{F}$ & F & $\mathrm{F}$ & $\mathrm{F}$ & d) & & & & & & & & & & & & & $\mathrm{O}$ \\
\hline I & $\mathrm{F}$ & $F$ & $\mathrm{~F}$ & $F$ & $\mathrm{~F}$ & S & S & & & & & & & & & & & & & 0 \\
\hline & B & B & B & B & B & B & B & B & B & B & B & B & B & B & B & B & B & B & B & \\
\hline
\end{tabular}

Figura 3.1: Célula (a) e domínio computacional (b) utilizadas pelo Freeflow3D.

aproximados pelo método upwind de alta ordem CUBISTA [3]. Assim, as velocidades $\widetilde{u}, \widetilde{v}, \widetilde{w}$ são calculadas pelas equações de diferenças finitas

$$
\begin{aligned}
& \widetilde{u}_{i+\frac{1}{2}, j, k}^{n+1}=u_{i+\frac{1}{2}, j, k}^{n}+\delta t\left\{-\left.\mathcal{C}(u u)\right|_{i+\frac{1}{2}, j, k}-\left.\mathcal{C}(v u)\right|_{i+\frac{1}{2}, j, k}-\left.\mathcal{C}(w u)\right|_{i+\frac{1}{2}, j, k}\right. \\
& -\left(\frac{\widetilde{p}_{i+1, j, k}-\widetilde{p}_{i, j, k}}{\delta x}\right)+\frac{1}{R e}\left[\frac{u_{i+\frac{3}{2}, j, k}-2 u_{i+\frac{1}{2}, j, k}+u_{i-\frac{1}{2}, j, k}}{\delta x^{2}}\right. \\
& +\frac{u_{i+\frac{1}{2}, j+1, k}-2 u_{i+\frac{1}{2}, j, k}+u_{i+\frac{1}{2}, j-1, k}}{\delta y^{2}}+\frac{u_{i+\frac{1}{2}, j, k+1}-2 u_{i+\frac{1}{2}, j, k}+u_{i+\frac{1}{2}, j, k-1}}{\delta z^{2}} \\
& \left.+\frac{\Phi_{i+1, j, k}^{x x}-\Phi_{i, j, k}^{x x}}{\delta x}+\frac{\Phi_{i+\frac{1}{2}, j+\frac{1}{2}, k}^{x y}-\Phi_{i+\frac{1}{2}, j-\frac{1}{2}, k}^{x y}}{\delta y}+\frac{\Phi_{i+\frac{1}{2}, j, k+\frac{1}{2}}^{x z}-\Phi_{i+\frac{1}{2}, j, k-\frac{1}{2}}^{x z}}{\delta z}\right] \\
& \left.+\frac{1}{F r^{2}} g_{x}\right\} \\
& \widetilde{v}_{i, j+\frac{1}{2}, k}^{n+1}=v_{i, j+\frac{1}{2}, k}^{n}+\delta t\left\{-\left.\mathcal{C}(u v)\right|_{i, j+\frac{1}{2}, k}-\left.\mathcal{C}(v v)\right|_{i, j+\frac{1}{2}, k}-\left.\mathcal{C}(w v)\right|_{i, j+\frac{1}{2}, k}\right. \\
& -\left(\frac{\widetilde{p}_{i, j+1, k}-\widetilde{p}_{i, j, k}}{\delta y}\right)+\frac{1}{R e}\left[\frac{v_{i+1, j+\frac{1}{2}, k}-2 v_{i, j+\frac{1}{2}, k}+v_{i-1, j+\frac{1}{2}, k}}{\delta x^{2}}\right. \\
& +\frac{v_{i, j+\frac{3}{2}, k}-2 v_{i, j+\frac{1}{2}, k}+v_{i, j-\frac{1}{2}, k}}{\delta y^{2}}+\frac{v_{i, j+\frac{1}{2}, k+1}-2 v_{i, j+\frac{1}{2}, k}+v_{i, j+\frac{1}{2}, k-1}}{\delta z^{2}} \\
& \left.+\frac{\Phi_{i+\frac{1}{2}, j+\frac{1}{2}, k}^{x y}-\Phi_{i-\frac{1}{2}, j+\frac{1}{2}, k}^{x y}}{\delta x}+\frac{\Phi_{i, j+1, k}^{y y}-\Phi_{i, j, k}^{y y}}{\delta y}+\frac{\Phi_{i, j+\frac{1}{2}, k+\frac{1}{2}}^{y z}-\Phi_{i, j+\frac{1}{2}, k-\frac{1}{2}}^{y z}}{\delta z}\right] \\
& \left.+\frac{1}{F r^{2}} g_{y}\right\}
\end{aligned}
$$




$$
\begin{aligned}
& \widetilde{w}_{i, j, k+\frac{1}{2}}^{n+1}=w_{i, j, k+\frac{1}{2}}^{n}+\delta t\left\{-\left.\mathcal{C}(u w)\right|_{i, j, k+\frac{1}{2}}-\left.\mathcal{C}(v w)\right|_{i, j, k+\frac{1}{2}}-\left.\mathcal{C}(w w)\right|_{i, j, k+\frac{1}{2}}\right. \\
& -\left(\frac{\widetilde{p}_{i+1, j, k}-\widetilde{p}_{i, j, k}}{\delta z}\right)+\frac{1}{R e}\left[\frac{w_{i+1, j, k+\frac{1}{2}}-2 w_{i, j, k+\frac{1}{2}}+w_{i-1, j, k+\frac{1}{2}}}{\delta x^{2}}\right. \\
& +\frac{w_{i, j+1, k+\frac{1}{2}}-2 w_{i, j, k+\frac{1}{2}}+w_{i, j-1, k+\frac{1}{2}}}{\delta y^{2}}+\frac{w_{i, j, k+\frac{3}{2}}-2 w_{i, j, k+\frac{1}{2}}+w_{i, j, k-\frac{1}{2}}}{\delta z^{2}} \\
& \left.+\frac{\Phi_{i+\frac{1}{2}, j, k+\frac{1}{2}}^{x z}-\Phi_{i-\frac{1}{2}, j, k+\frac{1}{2}}^{x z}}{\delta x}+\frac{\Phi_{i, j+\frac{1}{2}, k+\frac{1}{2}}^{y z}-\Phi_{i, j-\frac{1}{2}, k+\frac{1}{2}}^{y z}}{\delta y}+\frac{\Phi_{i, j, k+1}^{z z}-\Phi_{i, j, k}^{z z}}{\delta z}\right] \\
& \left.+\frac{1}{F r^{2}} g_{z}\right\}
\end{aligned}
$$

respectivamente. Nas equações acima, os termos $\left.\mathcal{C}(u u)\right|_{i+\frac{1}{2}, j, k}, \cdots,\left.\mathcal{C}(w w)\right|_{i, j, k+\frac{1}{2}}$ representam aproximações para os termos advectivos $\frac{\partial(u u)}{\partial x}, \cdots, \frac{\partial(w w)}{\partial z}$. Essas aproximações são calculadas pelo método upwind de alta ordem CUBISTA [3]. Detalhes sobre a implementação desse método para escoamentos cartesianos tridimensionais podem ser encontrados em Costacurta [18].

Nas equações acima, os termos que não estão definidos nas células são obtidos fazendo uma média aritmética utilizando valores vizinhos, por exemplo, $\Phi_{i+\frac{1}{2}, j+\frac{1}{2}, k}^{x y}$ e $\Phi_{i+\frac{1}{2}, j-\frac{1}{2}, k}^{x y}$ são obtidos por

$$
\begin{aligned}
\Phi_{i+\frac{1}{2}, j+\frac{1}{2}, k}^{x y} & =\frac{\Phi_{i, j, k}^{x y}+\Phi_{i+1, j, k}^{x y}+\Phi_{i, j+1, k}^{x y}+\Phi_{i+1, j+1, k}^{x y}}{4}, \\
\Phi_{i+\frac{1}{2}, j-\frac{1}{2}, k}^{x y} & =\frac{\Phi_{i, j, k}^{x y}+\Phi_{i+1, j, k}^{x y}+\Phi_{i, j-1, k}^{x y}+\Phi_{i+1, j-1, k}^{x y}}{4} .
\end{aligned}
$$

\subsubsection{Aproximações de segunda ordem}

Em células que estão próximas ao contorno da malha, as derivadas cruzadas da função reológica nas equações (3.1)-(3.3) são aproximadas por diferenças finitas de segunda ordem. Por exemplo, com relação a Figura 3.2, a derivada $\left.\frac{\partial \Phi^{x y}}{\partial y}\right|_{i+\frac{1}{2}, j, k}$ é obtida derivando-se o polinômio interpolador de $\Phi^{x y}$ nos pontos $\left(i+\frac{1}{2}, j, k\right),\left(i+\frac{1}{2}, j+1, k\right),\left(i+\frac{1}{2}, j+2, k\right)$ no ponto $\left(i+\frac{1}{2}, j, k\right)$. Nesse caso, obtém-se a seguinte fórmula

$$
\left.\frac{\partial \Phi^{x y}}{\partial y}\right|_{i+\frac{1}{2}, j, k}=\frac{-3 \Phi_{i+\frac{1}{2}, j, k}^{x y}+4 \Phi_{i+\frac{1}{2}, j+1, k}^{x y}-\Phi_{i+\frac{1}{2}, j+2, k}^{x y}}{2 \delta y} .
$$

As expressões para as outras derivadas cruzadas das componentes de $\boldsymbol{\Phi}$ nas equações (3.1)-(3.3) são obtidas de maneira análoga.

\subsection{Discretização da equação de Poisson}

A equação de Poisson é discretizada no centro da célula utilizando-se o operador laplaciano discreto com 7 pontos:

$$
\begin{aligned}
\frac{\psi_{i+1, j, k}-2 \psi_{i, j, k}+\psi_{i-1, j, k}}{\delta x^{2}} & +\frac{\psi_{i, j+1, k}-2 \psi_{i, j, k}+\psi_{i, j-1, k}}{\delta y^{2}} \\
& +\frac{\psi_{i, j, k+1}-2 \psi_{i, j, k}+\psi_{i, j, k-1}}{\delta z^{2}}=\widetilde{D}_{i j}
\end{aligned}
$$




\section{Nível $k$}

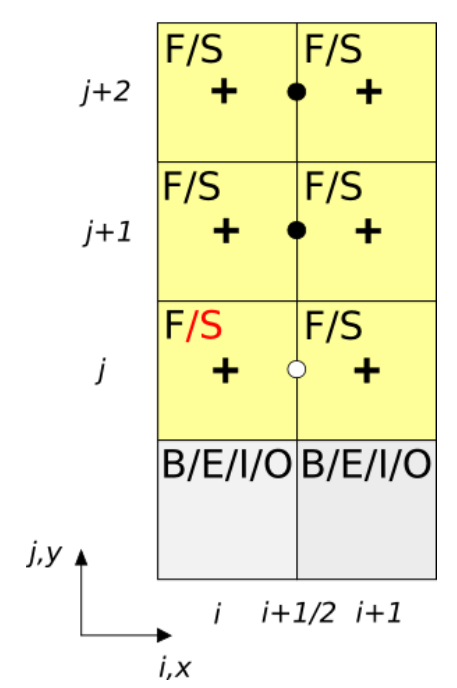

Figura 3.2: Pontos utilizados para o cálculo de $\left.\frac{\partial \Phi^{x y}}{\partial y}\right|_{i+\frac{1}{2}, j, k}$ por diferença de segunda ordem.

onde

$$
\widetilde{D}_{i j}=\frac{\widetilde{u}_{i+\frac{1}{2}, j, k}-\widetilde{u}_{i-\frac{1}{2}, j, k}}{\delta x}+\frac{\widetilde{v}_{i, j+\frac{1}{2}, k}-\widetilde{v}_{i, j-\frac{1}{2}, k}}{\delta y}+\frac{\widetilde{w}_{i, j, k+\frac{1}{2}}-\widetilde{w}_{i, j, k-\frac{1}{2}}}{\delta z}
$$

\subsection{Velocidades finais}

A velocidade final é obtida discretizando-se as equações (2.7) nos respectivos nós, ou seja,

$$
\begin{aligned}
& u_{i+\frac{1}{2}, j, k}^{n+1}=\widetilde{u}_{i+\frac{1}{2}, j, k}^{n+1}-\left(\frac{\psi_{i+1, j, k}^{n+1}-\psi_{i, j, k}^{n+1}}{\delta x}\right), \\
& v_{i, j+\frac{1}{2}, k}^{n+1}=\widetilde{v}_{i, j+\frac{1}{2}, k}^{n+1}-\left(\frac{\psi_{i, j+1, k}^{n+1}-\psi_{i, j, k}^{n+1}}{\delta y}\right), \\
& w_{i, j, k+\frac{1}{2}}^{n+1}=\widetilde{w}_{i, j, k+\frac{1}{2}}^{n+1}-\left(\frac{\psi_{i, j, k+1}^{n+1}-\psi_{i, j, k}^{n+1}}{\delta z}\right) .
\end{aligned}
$$

\subsection{Discretização da função reológica $\Phi$}

As componentes da função reológica são calculadas pelas equações (1.64)-(1.69), escritas na forma adimensional (ver 1.77). Por simplicidade, na discretização desta função, a derivada temporal do tensor taxa de deformação $\left(\frac{\partial \mathbf{D}}{\partial t}\right)$ foi desprezada, pois é supostamente pequena. 
Por exemplo, a componente $\Phi^{x x}$ é discretizada no centro da célula como

$$
\begin{aligned}
\Phi_{i, j, k}^{x x}= & D e\left\{\left.\frac{\partial\left(u \mathrm{D}^{x x}\right)}{\partial x}\right|_{i, j, k}+\left.\frac{\partial\left(v \mathrm{D}^{x x}\right)}{\partial y}\right|_{i, j, k}+\left.\frac{\partial\left(w \mathrm{D}^{x x}\right)}{\partial z}\right|_{i, j, k}\right. \\
& \left.-2\left[\left.\left(\frac{\partial u}{\partial x} \mathrm{D}^{x x}\right)\right|_{i, j, k}+\left.\left(\frac{\partial u}{\partial y} \mathrm{D}^{x y}\right)\right|_{i, j, k}+\left.\left(\frac{\partial u}{\partial z} \mathrm{D}^{x z}\right)\right|_{i, j, k}\right]\right\} \\
& +\kappa\left[\left.\left(\mathrm{D}^{x x}\right)^{2}\right|_{i, j, k}+\left.\left(\mathrm{D}^{x y}\right)^{2}\right|_{i, j, k}+\left.\left(\mathrm{D}^{x z}\right)^{2}\right|_{i, j, k}\right],
\end{aligned}
$$

onde os termos convectivos (por ex. $\left.\frac{\partial\left(u \mathrm{D}^{x x}\right)}{\partial x}\right|_{i, j, k}$ ) são aproximados pelo método de alta ordem CUBISTA [3]. As equações referentes as outras componentes $\Phi^{x y}, \Phi^{x z}, \ldots, \Phi^{z z}$ são apresentadas no Apêndice A.

As componentes do tensor taxa de deformação são compostas por derivadas parciais das componentes do vetor velocidade conforme equação (1.13). Essas derivadas são discretizadas usando diferenças centrais ou diferenças avançadas ou atrasadas. Por exemplo, $\frac{\partial u}{\partial x}$ é aproximada por

$$
\left.\frac{\partial u}{\partial x}\right|_{i, j, k} \approx \frac{u_{i+\frac{1}{2}, j, k}-u_{i-\frac{1}{2}, j, k}}{\delta x}
$$

e $\left.\frac{\partial v}{\partial y}\right|_{i, j, k},\left.\frac{\partial w}{\partial z}\right|_{i, j, k}$ são obtidas de maneira análoga. As derivadas cruzadas são obtidas por diferenças centrais ou de primeira ordem. Por exemplo, se uma célula F não tiver nenhuma face em contato com faces de células B ou E, então a derivada $\left.\frac{\partial u}{\partial y}\right|_{i, j, k}$ é aproximada por diferenças centrais

$$
\left.\frac{\partial u}{\partial y}\right|_{i, j, k} \approx \frac{u_{i, j+\frac{1}{2}, k}-u_{i, j-\frac{1}{2}, k}}{\delta y},
$$

onde

e

$$
u_{i, j+\frac{1}{2}, k}=\frac{u_{i+\frac{1}{2}, j, k}+u_{i-\frac{1}{2}, j, k}+u_{i+\frac{1}{2}, j+1, k}+u_{i-\frac{1}{2}, j+1, k}}{4}
$$

$$
u_{i, j-\frac{1}{2}, k}=\frac{u_{i+\frac{1}{2}, j, k}+u_{i-\frac{1}{2}, j, k}+u_{i+\frac{1}{2}, j-1, k}+u_{i-\frac{1}{2}, j-1, k}}{4} .
$$

Caso contrário, $\left.\frac{\partial u}{\partial y}\right|_{i, j, k}$ é aproximada por diferenças de primeira ordem. Por exemplo, se a célula $\mathrm{F}$ tiver a face $\left(i, j+\frac{1}{2}, k\right)$ em contato com uma face de célula $\mathrm{B}$, I ou O, então $\left.\frac{\partial u}{\partial y}\right|_{i, j, k}$ é aproximada por diferenças atrasadas

$$
\left.\frac{\partial u}{\partial y}\right|_{i, j, k} \approx \frac{u_{i, j, k}-u_{i, j-1, k}}{\delta y}
$$

onde

$$
u_{i, j, k}=\frac{u_{i+\frac{1}{2}, j, k}+u_{i-\frac{1}{2}, j, k}}{2} \quad \text { e } \quad u_{i, j-1, k}=\frac{u_{i+\frac{1}{2}, j-1, k}+u_{i-\frac{1}{2}, j-1, k}}{2} .
$$

Por outro lado, se a célula $\mathrm{F}$ tiver a face $\left(i, j-\frac{1}{2}, k\right)$ em contato com uma face de célula B, I ou O, então $\left.\frac{\partial u}{\partial y}\right|_{i, j, k}$ é aproximada por diferenças avançadas

$$
\left.\frac{\partial u}{\partial y}\right|_{i, j, k} \approx \frac{u_{i, j+1, k}-u_{i, j, k}}{\delta y}
$$

onde

$$
u_{i, j+1, k}=\frac{u_{i+\frac{1}{2}, j+1, k}+u_{i-\frac{1}{2}, j+1, k}}{2} \quad \text { e } \quad u_{i, j, k}=\frac{u_{i+\frac{1}{2}, j, k}+u_{i-\frac{1}{2}, j, k}}{2}
$$



análoga.

Os outros tipos de derivadas cruzadas $\left(\left.\frac{\partial u}{\partial z}\right|_{i, j, k},\left.\frac{\partial v}{\partial x}\right|_{i, j, k}, \cdots,\left.\frac{\partial w}{\partial y}\right|_{i, j, k}\right)$ são obtidos de maneira

\subsubsection{Aproximações de segunda ordem}

Em células $\mathrm{F}$ que estão em contato com faces de células B ou E, as derivadas cruzadas da velocidade na equação (3.11) podem ser calculadas por diferenças de segunda ordem. Essas derivadas são obtidas de maneira análoga ao procedimento utilizado para calcular as derivadas cruzadas das componentes do tensor $\boldsymbol{\Phi}$ nas equações (3.1)-(3.3). Por exemplo, com referência a Figura 3.3, a derivada $\left.\frac{\partial u}{\partial y}\right|_{i, j, k}$ é aproximada por

$$
\left.\frac{\partial u}{\partial y}\right|_{i, j, k} \approx \frac{-3 u_{i, j, k}+4 u_{i, j+1, k}-u_{i, j+2, k}}{2 \delta y}
$$

onde

$u_{i, j, k}=\frac{u_{i+\frac{1}{2}, j, k}+u_{i-\frac{1}{2}, j, k}}{2}, u_{i, j+1, k}=\frac{u_{i+\frac{1}{2}, j+1, k}+u_{i-\frac{1}{2}, j+1, k}}{2}, u_{i, j+2, k}=\frac{u_{i+\frac{1}{2}, j+2, k}+u_{i-\frac{1}{2}, j+2, k}}{2}$.

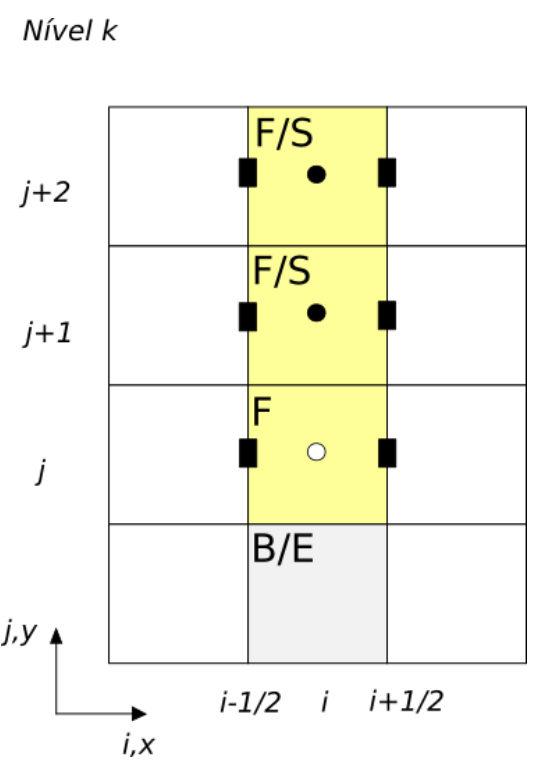

Figura 3.3: Pontos utilizados para o cálculo de $\left.\frac{\partial u}{\partial y}\right|_{i, j, k}$.

\subsection{Aplicação das condições de contorno na superfície livre}

Para aplicar as condições de contorno na superfície livre, conforme equações (1.82)-(1.84), vamos supor que o espaçamento da malha é suficientemente pequeno de modo que, localmente, a superfície livre possa ser aproximada por uma superfície plana, como segue.

\subsubsection{Superfície plana paralela a um eixo de coordenadas}

Essa superfície será definida como aquela em que o vetor normal unitário é paralelo a um dos eixos coordenados, isto é, $\mathbf{n}=( \pm 1,0,0)$ ou $\mathbf{n}=(0, \pm 1,0)$ ou $\mathbf{n}=(0,0, \pm 1)$. Estas 


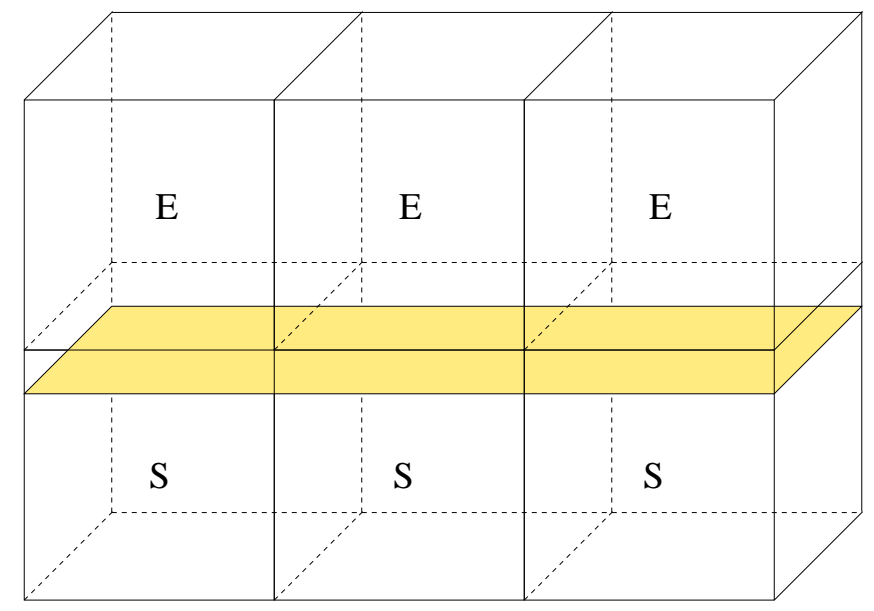

Figura 3.4: Células de superfície com somente uma face em contato com células vazias.

superfícies são identificadas por células de superfície livre S que possuem somente uma face em contato com uma face de célula vazia E (ver Figura 3.4).

Considerando as células $\mathrm{S}$ da Figura 3.4, o vetor normal toma a forma $\mathbf{n}=(0,0,1)$ e as equações (1.82)-(1.84) então se reduzem a

$$
\begin{gathered}
\widetilde{p}=\frac{1}{R e}\left(2 \frac{\partial w}{\partial z}+\Phi^{z z}\right), \\
\left(\frac{\partial v}{\partial z}+\frac{\partial w}{\partial y}\right)=-\Phi^{y z}, \\
\left(\frac{\partial u}{\partial z}+\frac{\partial w}{\partial x}\right)=-\Phi^{x z}
\end{gathered}
$$

respectivamente.

Por exemplo, considerando a célula $\mathrm{S}$ mostrada na Figura 3.5, quando calculamos a velocidade intermediária utilizando as equações (3.1)-(3.3), as velocidades $w_{i, j, k+\frac{1}{2}}, u_{i+\frac{1}{2}, j, k+1}$ e $v_{i, j+\frac{1}{2}, k+1}$ e a pressão $\widetilde{p}_{i, j, k}$ na célula de superfície livre são necessárias e obtidas como segue.

Discretizando (3.19) no ponto $\left(i+\frac{1}{2}, j, k+\frac{1}{2}\right)$ temos

$$
\frac{u_{i+\frac{1}{2}, j, k+1}-u_{i+\frac{1}{2}, j, k}}{\delta z}+\frac{w_{i+1, j, k+\frac{1}{2}}-w_{i, j, k+\frac{1}{2}}}{\delta x}=-\Phi_{i+\frac{1}{2}, j, k+\frac{1}{2}}^{x z}
$$

e discretizando também a equação (3.18) no ponto $\left(i, j+\frac{1}{2}, k+\frac{1}{2}\right)$ obtém-se

$$
\frac{v_{i, j+\frac{1}{2}, k+1}-v_{i, j+\frac{1}{2}, k}}{\delta z}+\frac{w_{i, j+1, k+\frac{1}{2}}-w_{i, j, k+\frac{1}{2}}}{\delta y}=-\Phi_{i, j+\frac{1}{2}, k+\frac{1}{2}}^{y z} .
$$

Agora, aplicando a equação da continuidade (1.75) no centro da célula S vem

$$
\frac{u_{i+\frac{1}{2}, j, k}-u_{i-\frac{1}{2}, j, k}}{\delta x}+\frac{v_{i, j+\frac{1}{2}, k}-v_{i, j-\frac{1}{2}, k}}{\delta y}+\frac{w_{i, j, k+\frac{1}{2}}-w_{i, j, k-\frac{1}{2}}}{\delta z}=0 .
$$




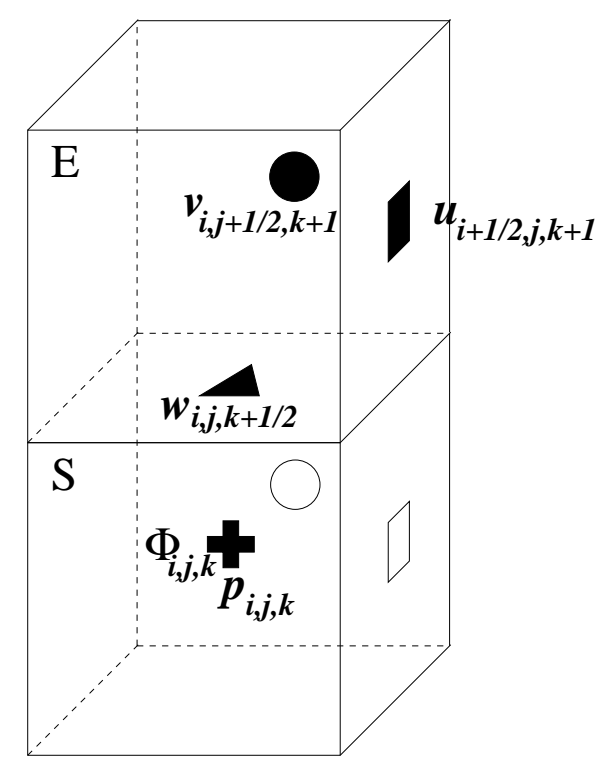

Figura 3.5: Célula S com somente a face $\left(k+\frac{1}{2}\right)$ em contato com células E.

As equações (3.20)-(3.22) fornecem 3 equações para as incógnitas $u_{i+\frac{1}{2}, j, k+1}, v_{i, j+\frac{1}{2}, k+1}$ e $w_{i, j, k+\frac{1}{2}}$ que podem ser resolvidas facilmente. Calculamos $w_{i, j, k+\frac{1}{2}}$ pela equação da continuidade $(3.22)$

$$
w_{i, j, k+\frac{1}{2}}=w_{i, j, k-\frac{1}{2}}-\frac{\delta z}{\delta x}\left(u_{i+\frac{1}{2}, j, k}-u_{i-\frac{1}{2}, j, k}\right)-\frac{\delta z}{\delta y}\left(v_{i, j+\frac{1}{2}, k}-v_{i, j-\frac{1}{2}, k}\right)
$$

e $u_{i+\frac{1}{2}, j, k+1}$ e $v_{i, j+\frac{1}{2}, k+1}$ pelas equações (3.20)-(3.21), ou seja,

$$
u_{i+\frac{1}{2}, j, k+1}=u_{i+\frac{1}{2}, j, k}-\frac{\delta z}{\delta x}\left(w_{i+1, j, k+\frac{1}{2}}-w_{i, j, k+\frac{1}{2}}\right)-\delta z \Phi_{i+\frac{1}{2}, j, k+\frac{1}{2}}^{x z}
$$

$\mathrm{e}$

$$
v_{i, j+\frac{1}{2}, k+1}=v_{i, j+\frac{1}{2}, k}-\frac{\delta z}{\delta y}\left(w_{i, j+1, k+\frac{1}{2}}-w_{i, j, k+\frac{1}{2}}\right)-\delta z \Phi_{i, j+\frac{1}{2}, k+\frac{1}{2}}^{y z}
$$

respectivamente.

A pressão $\widetilde{p}_{i, j, k}$ no centro da célula de superfície livre é então calculada usando (3.17) como

$$
\widetilde{p}_{i, j, k}=\frac{1}{R e}\left(\frac{2\left(w_{i, j, k+\frac{1}{2}}-w_{i, j, k-\frac{1}{2}}\right)}{\delta z}+\Phi_{i, j, k}^{z z}\right) .
$$

As demais configurações de células de superfície com apenas uma face em contato com faces de células vazias são tratadas de maneira similar e são detalhadas no Apêndice B.1.

\subsubsection{Superfície plana com inclinação de $45^{\circ}$}

Essas superfícies são identificadas por células de superfície que possuem duas faces adjacentes em contato com duas faces de células vazias. Nestas células assumimos que o vetor normal faz um ângulo de $45^{\circ}$ com dois eixos coordenados (ver Figura 3.6).

Por exemplo, consideremos a célula de superfície com as faces $\left(k+\frac{1}{2}\right)$ e $\left(i+\frac{1}{2}\right)$ em contato com duas faces adjacentes de células vazias conforme mostrado na Figura 3.7. Para 


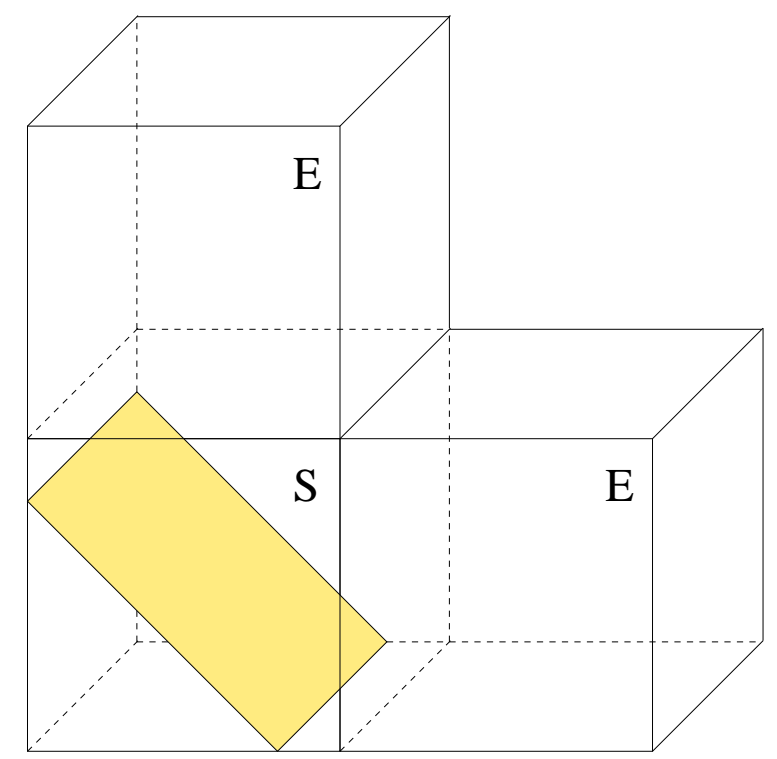

Figura 3.6: Células de superfície com duas faces adjacentes em contato com faces de células vazias.

esta célula assumimos que o vetor normal é dado por $\mathbf{n}=\left(\frac{\sqrt{2}}{2}, 0, \frac{\sqrt{2}}{2}\right)$ e os vetores tangenciais são:

$$
\mathbf{m} \mathbf{1}=\left(\frac{\sqrt{2}}{2}, 0,-\frac{\sqrt{2}}{2}\right) \quad \text { e } \mathbf{m} \mathbf{2}=(0,1,0) .
$$

Introduzindo esses vetores nas equações (1.82) e (1.83) obtemos

$$
\begin{gathered}
\widetilde{p}=\frac{1}{R e}\left[\frac{\partial u}{\partial x}+\frac{\partial w}{\partial z}+\left(\frac{\partial u}{\partial z}+\frac{\partial w}{\partial x}\right)+\frac{1}{2}\left(\Phi^{x x}+\Phi^{z z}\right)+\Phi^{x z}\right] \\
\frac{\partial u}{\partial x}-\frac{\partial w}{\partial z}=-\frac{1}{2}\left(\Phi^{x x}-\Phi^{z z}\right),
\end{gathered}
$$

respectivamente.

Com relação a Figura 3.7, os valores de $u_{i+\frac{1}{2}, j, k}$ e $w_{i, j, k+\frac{1}{2}}$ nas faces das células vazias são exigidos. Estes são obtidos pela equação (3.28) e a equação da continuidade (1.75) aplicadas no centro da célula de superfície livre. Nesse caso temos

$$
\frac{u_{i+\frac{1}{2}, j, k}-u_{i-\frac{1}{2}, j, k}}{\delta x}-\frac{w_{i, j, k+\frac{1}{2}}-w_{i, j, k-\frac{1}{2}}}{\delta z}=-\frac{1}{2}\left(\Phi_{i, j, k}^{x x}-\Phi_{i, j, k}^{z z}\right)
$$

e

$$
\frac{u_{i+\frac{1}{2}, j, k}-u_{i-\frac{1}{2}, j, k}}{\delta x}+\frac{w_{i, j, k+\frac{1}{2}}-w_{i, j, k-\frac{1}{2}}}{\delta z}=-\left(\frac{v_{i, j+\frac{1}{2}, k}-v_{i, j-\frac{1}{2}, k}}{\delta y}\right),
$$

respectivamente. Essas equações formam um sistema linear $2 \times 2$ para as velocidades $u_{i+\frac{1}{2}, j, k}$ e $w_{i, j, k+\frac{1}{2}}$ que tem como solução

$\mathrm{e}$

$$
u_{i+\frac{1}{2}, j, k}=u_{i-\frac{1}{2}, j, k}-\frac{1}{2} \frac{\delta x}{\delta y}\left(v_{i, j+\frac{1}{2}, k}-v_{i, j-\frac{1}{2}, k}\right)-\frac{\delta x}{4}\left(\Phi_{i, j, k}^{x x}-\Phi_{i, j, k}^{z z}\right)
$$

$$
w_{i, j, k+\frac{1}{2}}=w_{i, j, k-\frac{1}{2}}-\frac{1}{2} \frac{\delta z}{\delta y}\left(v_{i, j+\frac{1}{2}, k}-v_{i, j-\frac{1}{2}, k}\right)+\frac{\delta z}{4}\left(\Phi_{i, j, k}^{x x}-\Phi_{i, j, k}^{z z}\right) .
$$




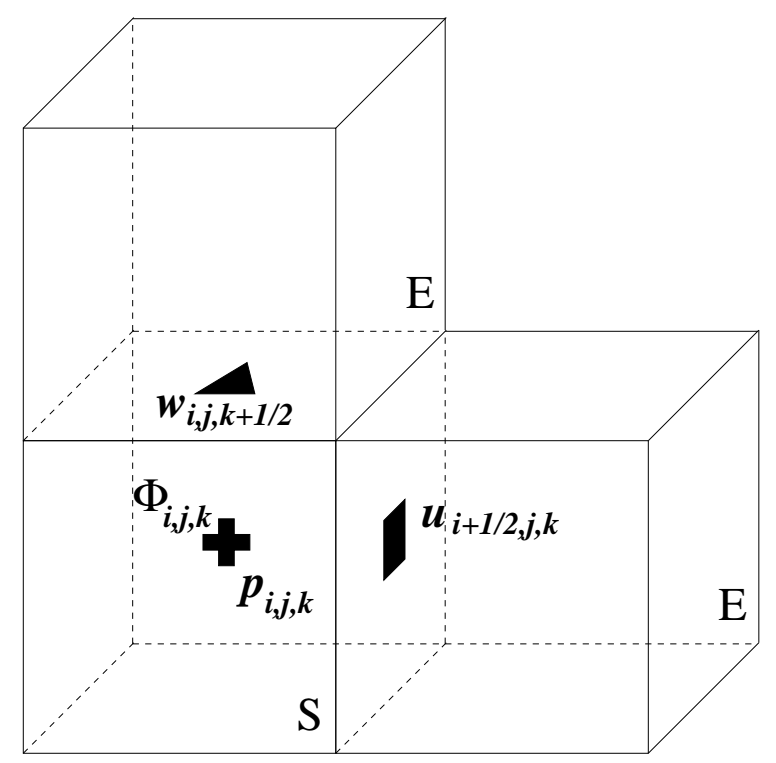

Figura 3.7: Célula S com as faces $\left(i+\frac{1}{2}\right)$ e $\left(k+\frac{1}{2}\right)$ em contato com faces de células vazias.

Tendo calculado as velocidades $u_{i+\frac{1}{2}, j, k}$ e $w_{i, j, k+\frac{1}{2}}$, a pressão no centro da célula de superfície livre é obtida por (3.27), ou seja,

$$
\begin{aligned}
\widetilde{p}_{i, j, k}= & \frac{1}{R e}\left[\frac{u_{i+\frac{1}{2}, j, k}-u_{i-\frac{1}{2}, j, k}}{\delta x}+\frac{w_{i, j, k+\frac{1}{2}}-w_{i, j, k-\frac{1}{2}}}{\delta z}\right. \\
& +\frac{1}{2}\left(\frac{u_{i+\frac{1}{2}, j, k}+u_{i-\frac{1}{2}, j, k}-u_{i+\frac{1}{2}, j, k-1}-u_{i-\frac{1}{2}, j, k-1}}{\delta z}\right. \\
& \left.+\frac{w_{i, j, k+\frac{1}{2}}+w_{i, j, k-\frac{1}{2}}-w_{i-1, j, k+\frac{1}{2}}-w_{i-1, j, k-\frac{1}{2}}}{\delta x}\right) \\
& \left.+\frac{1}{2}\left(\Phi_{i, j, k}^{x x}+\Phi_{i, j, k}^{z z}\right)+\Phi_{i, j, k}^{x z}\right] .
\end{aligned}
$$

Outras configurações de células de superfície com duas faces adjacentes em contato com faces de células vazias são tratadas de maneira semelhante e para detalhes ver Apêndice B.2.

\subsubsection{Superfície plana com inclinação de $60^{\circ}$}

Nessas superfícies assume-se que o vetor normal faz um ângulo de $60^{\circ} \mathrm{com}$ os eixos coordenados e são identificadas por células de superfície com três faces adjacentes em contato com células vazias (ver Figura 3.8). Por exemplo, considere a célula de superfície com as faces $\left(i+\frac{1}{2}\right),\left(j+\frac{1}{2}\right)$ e $\left(k+\frac{1}{2}\right)$ em contato com faces de células vazias como mostrada na Figura 3.9. Para essa célula tomamos como vetores unitários

$$
\mathbf{n}=\left(\frac{\sqrt{3}}{3}, \frac{\sqrt{3}}{3}, \frac{\sqrt{3}}{3}\right), \mathbf{m} \mathbf{1}=\left(\frac{\sqrt{2}}{2},-\frac{\sqrt{2}}{2}, 0\right), \mathbf{m} \mathbf{2}=\left(\frac{\sqrt{6}}{6}, \frac{\sqrt{6}}{6},-\frac{\sqrt{6}}{3}\right)
$$




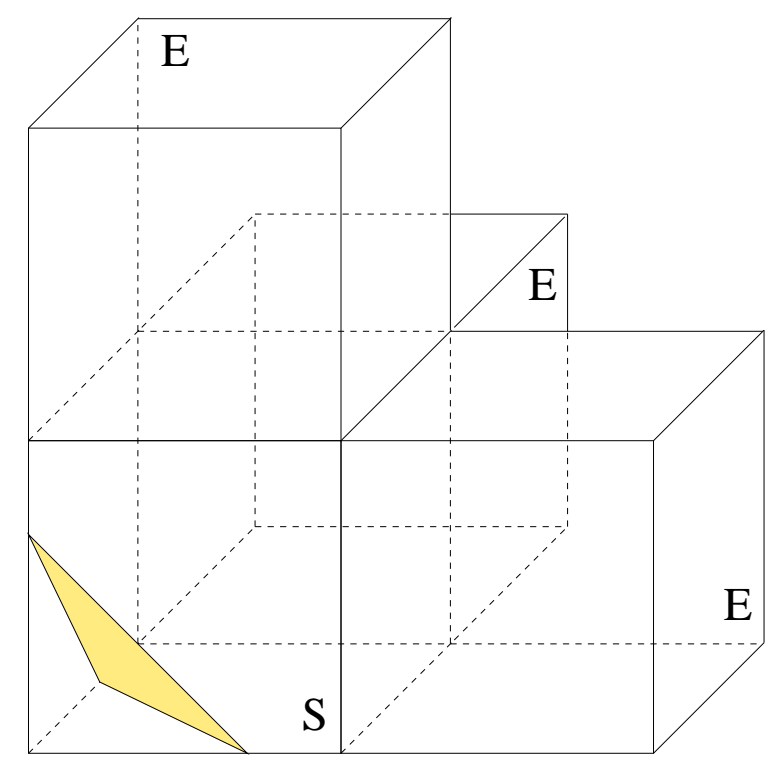

Figura 3.8: Células de superfície com três faces em contato com células vazias.

Introduzindo $\mathbf{n}, \mathbf{m} 1$ e $\mathbf{m} 2$ nas condições (1.82)-(1.84) obtemos

$$
\begin{gathered}
\widetilde{p}=\frac{1}{3 R e}\left[2\left(\frac{\partial u}{\partial y}+\frac{\partial v}{\partial x}\right)+2\left(\frac{\partial u}{\partial z}+\frac{\partial w}{\partial x}\right)+2\left(\frac{\partial v}{\partial z}+\frac{\partial w}{\partial y}\right)\right. \\
\left.+\Phi^{x x}+\Phi^{y y}+\Phi^{z z}+2\left(\Phi^{x y}+\Phi^{x z}+\Phi^{y z}\right)\right], \\
2 \frac{\partial u}{\partial x}-2 \frac{\partial v}{\partial y}+\left(\frac{\partial u}{\partial z}+\frac{\partial w}{\partial x}\right)-\left(\frac{\partial v}{\partial z}+\frac{\partial w}{\partial y}\right)=-\left[\Phi^{x x}-\Phi^{y y}+\Phi^{x z}-\Phi^{y z}\right], \\
2 \frac{\partial u}{\partial x}+2 \frac{\partial v}{\partial y}-4 \frac{\partial w}{\partial z}+2\left(\frac{\partial u}{\partial y}+\frac{\partial v}{\partial x}\right)-\left(\frac{\partial u}{\partial z}+\frac{\partial w}{\partial x}\right)-\left(\frac{\partial v}{\partial z}+\frac{\partial w}{\partial y}\right) \\
=-\left[\Phi^{x x}+\Phi^{y y}-2 \Phi^{z z}+2 \Phi^{x y}-\Phi^{x z}-\Phi^{y z}\right],
\end{gathered}
$$

respectivamente. A conservação da massa para estas células também requer

$$
\frac{\partial u}{\partial x}+\frac{\partial v}{\partial y}+\frac{\partial w}{\partial z}=0
$$

Agora, adicionando as equações (3.35) e (3.36) obtém-se

$$
2 \frac{\partial u}{\partial x}-2 \frac{\partial w}{\partial z}+\left(\frac{\partial u}{\partial y}+\frac{\partial v}{\partial x}\right)-\left(\frac{\partial v}{\partial z}+\frac{\partial w}{\partial y}\right)=\left[-\Phi^{x x}+\Phi^{z z}-\Phi^{x y}+\Phi^{y z}\right] .
$$

Com relação a Figura 3.9, os valores de $u_{i+\frac{1}{2}, j, k}, v_{i, j+\frac{1}{2}, k}, w_{i, j, k+\frac{1}{2}}$ e $\widetilde{p}_{i, j, k}$ são requeridos e podem ser calculados pelas equações $(3.35),(3.37),(3.38)$ e (3.34), como segue.

Aplicando a equação de conservação de massa no centro da célula, obtemos

$$
\frac{u_{i+\frac{1}{2}, j, k}-u_{i-\frac{1}{2}, j, k}}{\delta x}+\frac{v_{i, j+\frac{1}{2}, k}-v_{i, j-\frac{1}{2}, k}}{\delta y}+\frac{w_{i, j, k+\frac{1}{2}}-w_{i, j, k-\frac{1}{2}}}{\delta z}=0
$$




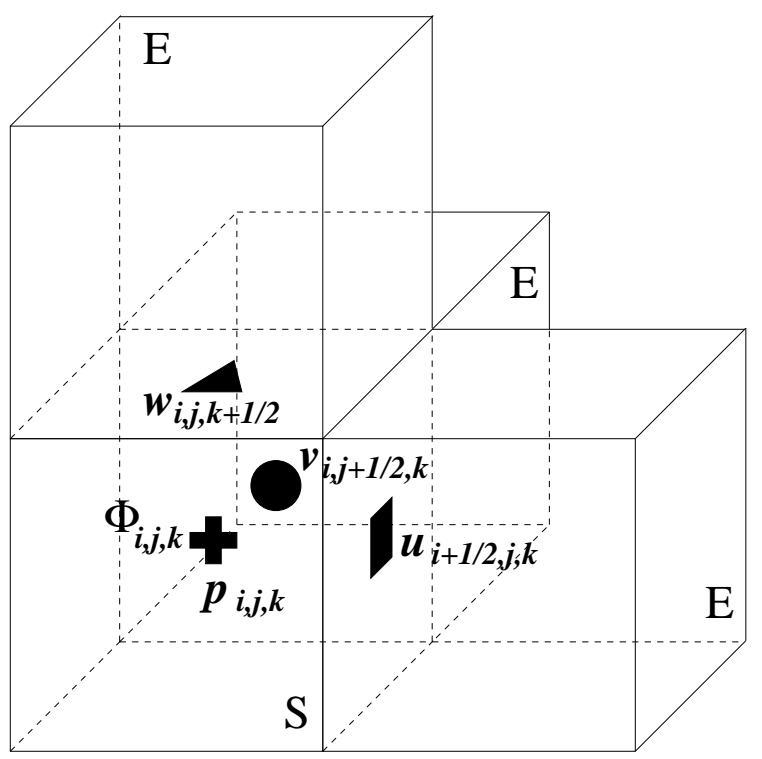

Figura 3.9: Célula S com as faces $\left(i+\frac{1}{2}\right),\left(j+\frac{1}{2}\right)$ e $\left(k+\frac{1}{2}\right)$ em contato com faces de células vazias.

que pode ser escrita como

$$
u_{i+\frac{1}{2}, j, k}+\frac{\delta x}{\delta y} v_{i, j+\frac{1}{2}, k}+\frac{\delta x}{\delta z} w_{i, j, k+\frac{1}{2}}=b_{3}
$$

onde

$$
b_{3}=u_{i-\frac{1}{2}, j, k}+\frac{\delta x}{\delta y} v_{i, j-\frac{1}{2}, k}+\frac{\delta x}{\delta z} w_{i, j, k-\frac{1}{2}} .
$$

Agora, a equação (3.35) aplicada no centro da célula fornece

$$
\begin{aligned}
& 2\left(\frac{u_{i+\frac{1}{2}, j, k}-u_{i-\frac{1}{2}, j, k}}{\delta x}\right)-2\left(\frac{v_{i, j+\frac{1}{2}, k}-v_{i, j-\frac{1}{2}, k}}{\delta y}\right) \\
& +\frac{1}{2}\left(\frac{u_{i+\frac{1}{2}, j, k}+u_{i-\frac{1}{2}, j, k}-u_{i+\frac{1}{2}, j, k-1}-u_{i-\frac{1}{2}, j, k-1}}{\delta z}\right. \\
& \left.+\frac{w_{i, j, k+\frac{1}{2}}+w_{i, j, k-\frac{1}{2}}-w_{i-1, j, k+\frac{1}{2}}-w_{i-1, j, k-\frac{1}{2}}}{\delta x}\right) \\
& -\frac{1}{2}\left(\frac{v_{i, j+\frac{1}{2}, k}+v_{i, j-\frac{1}{2}, k}-v_{i, j+\frac{1}{2}, k-1}-v_{i, j-\frac{1}{2}, k-1}}{\delta z}\right) \\
& \left.+\frac{w_{i, j, k+\frac{1}{2}}+w_{i, j, k-\frac{1}{2}}-w_{i, j-1, k+\frac{1}{2}}-w_{i, j-1, k-\frac{1}{2}}}{\delta y}\right) \\
& =-\left[\Phi_{i, j, k}^{x x}-\Phi_{i, j, k}^{y y}+\Phi_{i, j, k}^{x z}-\Phi_{i, j, k}^{y z}\right]
\end{aligned}
$$

que pode ser reescrita como (multiplicando por 2 e por $\delta x$ )

$$
\left(4+\frac{\delta x}{\delta z}\right) u_{i+\frac{1}{2}, j, k}-\left(4 \frac{\delta x}{\delta y}+\frac{\delta x}{\delta z}\right) v_{i, j+\frac{1}{2}, k}+\left(1-\frac{\delta x}{\delta y}\right) w_{i, j, k+\frac{1}{2}}=b_{2}
$$


onde

$$
\begin{aligned}
b_{2}= & 4 u_{i-\frac{1}{2}, j, k}-4 \frac{\delta x}{\delta y} v_{i, j-\frac{1}{2}, k}-\left(\frac{\delta x}{\delta z}\right)\left(u_{i-\frac{1}{2}, j, k}-u_{i+\frac{1}{2}, j, k-1}-u_{i-\frac{1}{2}, j, k-1}\right) \\
& -\left(w_{i, j, k-\frac{1}{2}}-w_{i-1, j, k+\frac{1}{2}}-w_{i-1, j, k-\frac{1}{2}}\right) \\
& +\left(\frac{\delta x}{\delta z}\right)\left(v_{i, j-\frac{1}{2}, k}-v_{i, j+\frac{1}{2}, k-1}-v_{i, j-\frac{1}{2}, k-1}\right) \\
& +\left(\frac{\delta x}{\delta y}\right)\left(w_{i, j, k-\frac{1}{2}}-w_{i, j-1, k+\frac{1}{2}}-w_{i, j-1, k-\frac{1}{2}}\right) \\
& -2 \delta x\left[\Phi_{i, j, k}^{x x}-\Phi_{i, j, k}^{y y}+\Phi_{i, j, k}^{x z}-\Phi_{i, j, k}^{y z}\right] .
\end{aligned}
$$

Analogamente, discretizando (3.38) da célula de superfície na posição $(i, j, k)$, obtemos

$$
\begin{aligned}
& 2\left(\frac{u_{i+\frac{1}{2}, j, k}-u_{i-\frac{1}{2}, j, k}}{\delta x}\right)-2\left(\frac{w_{i, j, k+\frac{1}{2}}-w_{i, j, k-\frac{1}{2}}}{\delta z}\right) \\
& +\frac{1}{2}\left(\frac{u_{i+\frac{1}{2}, j, k}+u_{i-\frac{1}{2}, j, k}-u_{i+\frac{1}{2}, j-1, k}-u_{i-\frac{1}{2}, j-1, k}}{\delta y}\right. \\
& \left.+\frac{v_{i, j+\frac{1}{2}, k}+v_{i, j-\frac{1}{2}, k}-v_{i-1, j+\frac{1}{2}, k}-v_{i-1, j-\frac{1}{2}, k}}{\delta x}\right) \\
& -\frac{1}{2}\left(\frac{v_{i, j+\frac{1}{2}, k}+v_{i, j-\frac{1}{2}, k}-v_{i, j+\frac{1}{2}, k-1}-v_{i, j-\frac{1}{2}, k-1}}{\delta z}\right) \\
& \left.+\frac{w_{i, j, k+\frac{1}{2}}+w_{i, j, k-\frac{1}{2}}-w_{i, j-1, k+\frac{1}{2}}-w_{i, j-1, k-\frac{1}{2}}}{\delta y}\right) \\
& =\left[-\Phi_{i, j, k}^{x x}+\Phi_{i, j, k}^{z z}-\Phi_{i, j, k}^{x y}+\Phi_{i, j, k}^{y z}\right]
\end{aligned}
$$

a qual reescrevemos como

$$
\left(4+\frac{\delta x}{\delta y}\right) u_{i+\frac{1}{2}, j, k}+\left(1-\frac{\delta x}{\delta z}\right) v_{i, j+\frac{1}{2}, k}-\left(4 \frac{\delta x}{\delta z}+\frac{\delta x}{\delta y}\right) w_{i, j, k+\frac{1}{2}}=b_{1},
$$

onde

$$
\begin{aligned}
b_{1}= & 4 u_{i-\frac{1}{2}, j, k}-4 \frac{\delta x}{\delta z} w_{i, j, k-\frac{1}{2}}-\left(\frac{\delta x}{\delta y}\right)\left(u_{i-\frac{1}{2}, j, k}-u_{i+\frac{1}{2}, j-1, k}-u_{i-\frac{1}{2}, j-1, k}\right) \\
& -\left(v_{i, j-\frac{1}{2}, k}-v_{i-1, j+\frac{1}{2}, k}-v_{i-1, j-\frac{1}{2}, k}\right) \\
& +\left(\frac{\delta x}{\delta z}\right)\left(v_{i, j-\frac{1}{2}, k}-v_{i, j+\frac{1}{2}, k-1}-v_{i, j-\frac{1}{2}, k-1}\right) \\
& +\left(\frac{\delta x}{\delta y}\right)\left(w_{i, j, k-\frac{1}{2}}-w_{i, j-1, k+\frac{1}{2}}-w_{i, j-1, k-\frac{1}{2}}\right) \\
& +2 \delta x\left[-\Phi_{i, j, k}^{x x}+\Phi_{i, j, k}^{z z}-\Phi_{i, j, k}^{x y}+\Phi_{i, j, k}^{y z}\right] .
\end{aligned}
$$

As equações (3.47), (3.44) e (3.40) fornecem um sistema linear $(3 \times 3)$ para as velocidades $u_{i+\frac{1}{2}, j, k}, v_{i, j+\frac{1}{2}, k}, w_{i, j, k+\frac{1}{2}}$ que pode ser escrito na forma matricial como:

$$
\left[\begin{array}{ccc}
\left(4+\frac{\delta x}{\delta y}\right) & \left(1-\frac{\delta x}{\delta z}\right) & -\left(4 \frac{\delta x}{\delta z}+\frac{\delta x}{\delta y}\right) \\
\left(4+\frac{\delta x}{\delta z}\right) & -\left(4 \frac{\delta x}{\delta y}+\frac{\delta x}{\delta z}\right) & \left(1-\frac{\delta x}{\delta y}\right) \\
1 & \frac{\delta x}{\delta y} & \frac{\delta x}{\delta z}
\end{array}\right]\left[\begin{array}{c}
u_{i+\frac{1}{2}, j, k} \\
v_{i, j+\frac{1}{2}, k} \\
w_{i, j, k+\frac{1}{2}}
\end{array}\right]=\left[\begin{array}{c}
b_{1} \\
b_{2} \\
b_{3}
\end{array}\right]
$$


Esse sistema pode ser resolvido facilmente pelo método de eliminação de Gauss. Com os valores de $u_{i+\frac{1}{2}, j, k}, v_{i, j+\frac{1}{2}, k}$ e $w_{i, j, k+\frac{1}{2}}$ calculados a pressão é obtida pela discretização de (3.34), no centro da célula de superfície, obtendo,

$$
\begin{aligned}
\widetilde{p}_{i, j, k}= & \frac{1}{3 R e}\left[\left(\frac{u_{i+\frac{1}{2}, j, k}+u_{i-\frac{1}{2}, j, k}-u_{i+\frac{1}{2}, j-1, k}-u_{i-\frac{1}{2}, j-1, k}}{\delta y}\right.\right. \\
& \left.+\frac{v_{i, j+\frac{1}{2}, k}+v_{i, j-\frac{1}{2}, k}-v_{i-1, j+\frac{1}{2}, k}-v_{i-1, j-\frac{1}{2}, k}}{\delta x}\right) \\
& +\left(\frac{u_{i+\frac{1}{2}, j, k}+u_{i-\frac{1}{2}, j, k}-u_{i+\frac{1}{2}, j, k-1}-u_{i-\frac{1}{2}, j, k-1}}{\delta z}\right. \\
& \left.+\frac{w_{i, j, k+\frac{1}{2}}+w_{i, j, k-\frac{1}{2}}-w_{i-1, j, k+\frac{1}{2}}-w_{i-1, j, k-\frac{1}{2}}}{\delta x}\right) \\
& +\left(\frac{v_{i, j+\frac{1}{2}, k}+v_{i, j-\frac{1}{2}, k}-v_{i, j+\frac{1}{2}, k-1}-v_{i, j-\frac{1}{2}, k-1}}{\delta z}\right. \\
& \left.\left.+\frac{w_{i, j, k+\frac{1}{2}}+w_{i, j, k-\frac{1}{2}}-w_{i, j-1, k+\frac{1}{2}}-w_{i, j-1, k-\frac{1}{2}}}{\delta y}\right)\right] \\
& +\frac{1}{3 R e}\left[\Phi_{i, j, k}^{x x}+\Phi_{i, j, k}^{y y}+\Phi_{i, j, k}^{z z}+2\left(\Phi_{i, j, k}^{x y}+\Phi_{i, j, k}^{x z}+\Phi_{i, j, k}^{y z}\right)\right] .
\end{aligned}
$$

As demais configurações de células de superfície com três faces adjacentes em contato com faces de células vazias são tratadas de maneira similar e são detalhadas no Apêndice B.3.

\subsection{Cálculo do passo no tempo $(\delta t)$}

Um procedimento para otimizar o tamanho do passo no tempo em cada ciclo de cálculo é utilizado. O tamanho do passo no tempo é calculado segundo as seguintes restrições:

(a) Nenhuma partícula pode cruzar mais do que uma célula em um dado intervalo de tempo, ou seja,

$$
\delta t<\frac{h}{|\mathbf{u}|}(\text { componente a componente) }
$$

onde $h=\min \{\delta x, \delta y, \delta z\}$.

(b) Devido à discretização explícita da equação de conservação de quantidade de movimento, adota-se a seguinte restrição de estabilidade que envolve o número de Reynolds:

$$
\left\{\begin{array}{l}
\delta t=0.25 R e h^{2}, \text { se } R e<1 \\
\delta t=0.25 h^{2}, \text { caso contrário. }
\end{array}\right.
$$

Para satisfazer (3.50) é suficiente que:

$$
\delta t<\frac{h}{\left|U_{\max }\right|},
$$

onde $U_{\max }$ representa o valor máximo de $\mathbf{u}$.

A implementação dessas condições segue as mesmas normas como implementado no método GENSMAC3D [19]. 
CAPÍTULO

\section{Validação do método numérico}

Com o objetivo de simular escoamentos tridimensionais governados pela equação constitutiva Fluido de Segunda Ordem, as equações de diferenças finitas desenvolvidas no Capítulo 3 foram implementadas no sistema de simulação Freeflow3D [17]. Uma validação e resultados de convergência do método numérico desenvolvido nesse trabalho são apresentados a seguir.

\subsection{Escoamento totalmente desenvolvido em um tubo}

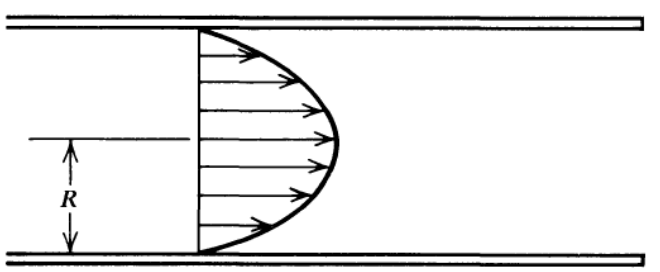

(a) Perfil parabólico

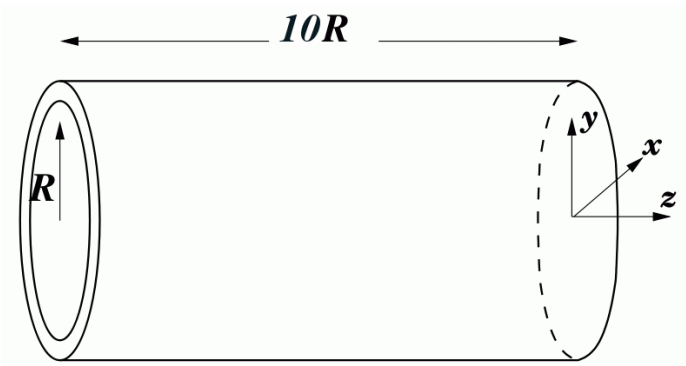

(b) Dimensões do tubo

Figura 4.1: Representação do escoamento em um tubo.

Como o tensor das tensões extra é obtido explicitamente em função do vetor velocidade, então, se considerarmos o escoamento totalmente desenvolvido em um tubo (ver Figura 4.1(b)) podemos facilmente obter a solução analítica para o tensor não-Newtoniano $\Phi$ conforme a equação (1.62). De fato, se supormos escoamento totalmente desenvolvido com

$$
\frac{\partial p}{\partial z}=P(\text { constante }), \quad \mathbf{u}=(0,0, w(x, y))
$$


então, pode ser verificado que as componentes do tensor não-Newtoniano $\boldsymbol{\Phi}$ são dadas por

$$
\begin{aligned}
\Phi^{x x} & =\kappa\left(\frac{\partial w}{\partial x}\right)^{2} \\
\Phi^{x y} & =\kappa \frac{\partial w}{\partial x} \frac{\partial w}{\partial y} \\
\Phi^{x z} & =0 \\
\Phi^{y y} & =\kappa\left(\frac{\partial w}{\partial y}\right)^{2} \\
\Phi^{y z} & =0 \\
\Phi^{z z} & =(\kappa-2 D e)\left[\left(\frac{\partial w}{\partial x}\right)^{2}+\left(\frac{\partial w}{\partial y}\right)^{2}\right] .
\end{aligned}
$$

Introduzindo equações (4.2)-(4.7) na equação de conservação de quantidade de movimento (1.76), obtemos

$$
\begin{aligned}
& \frac{\partial p}{\partial x}=\frac{\kappa}{R e}\left[2 \frac{\partial w}{\partial x} \frac{\partial^{2} w}{\partial x^{2}}+\frac{\partial w}{\partial x} \frac{\partial^{2} w}{\partial y^{2}}+\frac{\partial w}{\partial y} \frac{\partial^{2} w}{\partial x \partial y}\right] \\
& \frac{\partial p}{\partial y}=\frac{\kappa}{R e}\left[\frac{\partial^{2} w}{\partial x^{2}} \frac{\partial w}{\partial y}+\frac{\partial w}{\partial x} \frac{\partial^{2} w}{\partial x \partial y}+2 \frac{\partial w}{\partial y} \frac{\partial^{2} w}{\partial y^{2}}\right] \\
& \frac{\partial p}{\partial z}=\frac{1}{R e}\left[\frac{\partial^{2} w}{\partial x^{2}}+\frac{\partial^{2} w}{\partial y^{2}}\right] .
\end{aligned}
$$

Agora, fazendo $w(x, y)=\mathcal{A}\left(x^{2}+y^{2}\right)+\mathcal{B}$, pode ser verificado que as componentes do tensor não-Newtoniano $\boldsymbol{\Phi}$ são dadas por

$$
\begin{aligned}
\Phi^{x x} & =4 \kappa \mathcal{A}^{2} x^{2} \\
\Phi^{x y} & =4 \kappa \mathcal{A}^{2} x y \\
\Phi^{x z} & =0 \\
\Phi^{y y} & =4 \kappa \mathcal{A}^{2} y^{2} \\
\Phi^{y z} & =0 \\
\Phi^{z z} & =4(\kappa-2 D e) \mathcal{A}^{2}\left[x^{2}+y^{2}\right]
\end{aligned}
$$

e equações (4.8)-(4.10) se reduzem a

$$
\begin{aligned}
& \frac{\partial p}{\partial x}=12 \mathcal{A}^{2} \frac{\kappa}{R e} x, \\
& \frac{\partial p}{\partial y}=12 \mathcal{A}^{2} \frac{\kappa}{R e} y, \\
& \frac{\partial p}{\partial z}=4 \mathcal{A} \frac{1}{R e} .
\end{aligned}
$$

Integrando (4.17)-(4.19), obtém-se que a pressão é dada por

$$
p(x, y, z)=12 \mathcal{A}^{2} \frac{\kappa}{R e}\left(\frac{x^{2}}{2}+\frac{y^{2}}{2}\right)+\frac{4 \mathcal{A}}{R e} z+C \text { (constante) }
$$


Observamos que a solução apresentada pelas equações (4.11)-(4.16) são válidas em qualquer secção transversal do tubo.

Para validar o método numérico apresentado nesse trabalho simulamos o escoamento em um tubo (conforme mostrado na Figura 4.1(b)) até atingir o estado estacionário e comparamos as soluções numéricas com as respectivas soluções analíticas dadas pelas equações (4.11)-(4.16). Os dados (adimensionais) utilizados foram: $w(x, y)=\frac{2 U}{R^{2}}\left[R^{2}-\left(x^{2}+y^{2}\right)\right]$, $R=1, U=1, R e=1, D e=0.4, \kappa=0.4, g=0$ (gravidade não foi considerada). Para verificar a convergência do método numérico, esse problema foi simulado em três malhas:

- Malha M0 - $\delta x=\delta y=\delta z=0.16666667(12 \times 12 \times 60$ células computacionais $)$;

- Malha M1 $-\delta x=\delta y=\delta z=0.125(16 \times 16 \times 80$ células computacionais $)$;

- Malha M2 - $\delta x=\delta y=\delta z=0.1(20 \times 20 \times 100$ células computacionais $)$.

Na parede do tubo foi imposta a condição não-escorregamento e o fluido foi injetado na entrada do tubo até o mesmo ficar totalmente preenchido. Na entrada do tubo foi imposto escoamento Poiseuille especificado acima e na saída do tubo as condições homogêneas de Neumann foram adotadas. Inicialmente teve um escoamento transiente com superfície livre onde as condições de contorno na superfície livre foram aquelas apresentadas na seção 3.5. As simulações começaram com o tubo vazio $(t=0)$ e terminaram em $t=50$. A Figura 4.2 mostra o escoamento nos tempos $t=3,6,50$, enquanto que a Figura 4.3 mostra a variação da velocidade $w$ na secção transversal do tubo. Podemos ver na Figura 4.3 que as isolinhas estão paralelas, o que mostra que o escoamento está totalmente desenvolvido.

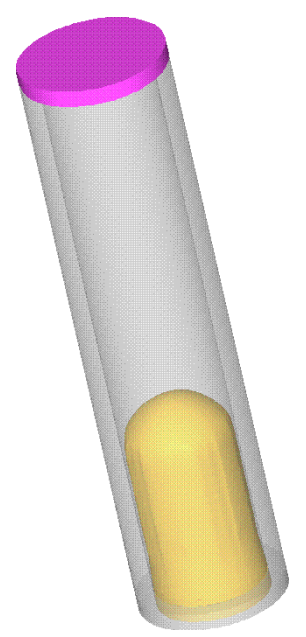

(a) $\mathrm{t}=3$

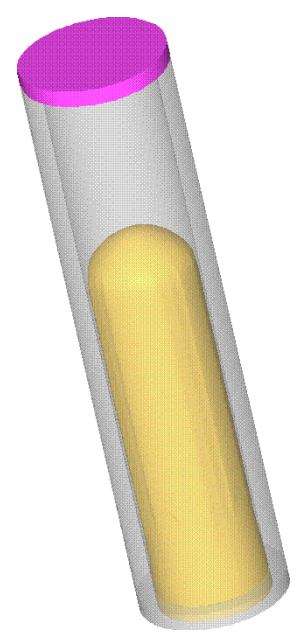

(b) $\mathrm{t}=6$

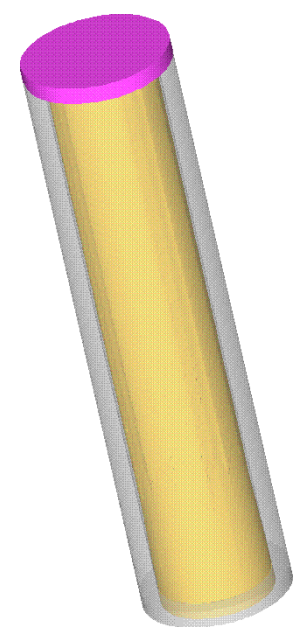

(c) $\mathrm{t}=50$

Figura 4.2: Enchimento do tudo com fluido de segunda ordem: $D e=0.4, \kappa=0.4$ e malha M2.

Para verificarmos a convergência do método numérico para resolver as equações governantes de Fluido de Segunda Ordem, comparamos as soluções numéricas com as respectivas soluções analíticas (conforme equações (4.11)-(4.16)) na secção transversal no meio do tubo $(z=5)$ nas três malhas. A Figura 4.4 mostra as soluções numéricas obtidas juntamente com as soluções analíticas nas três malhas, onde podemos ver que as soluções numéricas 


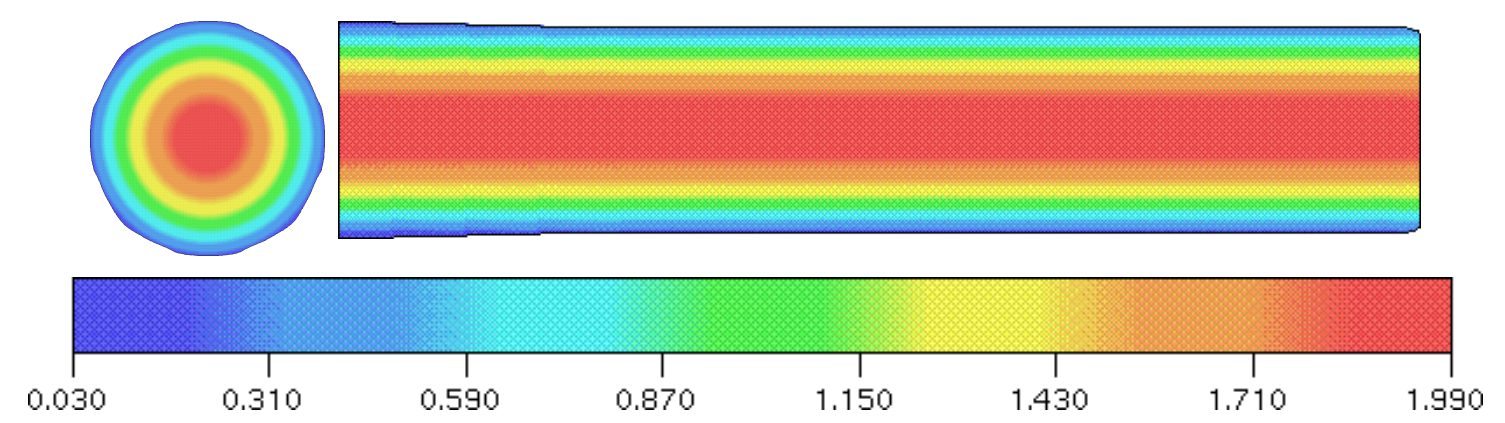

Figura 4.3: Visualização de $w(x, y)$ no meio e ao longo do tubo em $t=50$.

aproximam as soluções analíticas nas três malhas. Para quantificarmos esse fato, calculamos os erros relativos entre as soluções analíticas e as soluções numéricas pela fórmula

$$
E\left(S o l_{\mathrm{Num}}\right)=\sqrt{\frac{\sum_{i, j}\left(S o l_{\mathrm{Ex}}-S o l_{\mathrm{Num}}\right)^{2}}{\sum_{i, j}\left(S o l_{\mathrm{Ex}}\right)^{2}}}
$$

A tabela 4.1 mostra os erros obtidos nas três malhas, enquanto que a Figura 4.5 ilustra o decaimento dos erros em função das malhas utilizadas. Podemos ver na tabela 4.1 e na Figura 4.5 que os erros descrescem à medida que a malha é refinada, demonstrando assim, a convergência do método numérico apresentado nesse trabalho.

Tabela 4.1: Erros obtidos nas três malhas.

\begin{tabular}{|c|c|c|c|}
\hline Malha & M0 & M1 & M2 \\
\hline \hline$E(w(x, y))$ & $4.956 \times 10^{-3}$ & $2.696 \times 10^{-3}$ & $1.548 \times 10^{-3}$ \\
\hline$E\left(\Phi^{x x}(x, y)\right)$ & $2.309 \times 10^{-2}$ & $1.099 \times 10^{-2}$ & $7.166 \times 10^{-3}$ \\
\hline$E\left(\Phi^{x y}(x, y)\right)$ & $2.275 \times 10^{-2}$ & $1.082 \times 10^{-2}$ & $6.336 \times 10^{-3}$ \\
\hline$E\left(\Phi^{y y}(x, y)\right)$ & $2.321 \times 10^{-2}$ & $1.078 \times 10^{-2}$ & $7.704 \times 10^{-3}$ \\
\hline$E\left(\Phi^{z z}(x, y)\right)$ & $2.320 \times 10^{-2}$ & $1.037 \times 10^{-2}$ & $6.678 \times 10^{-3}$ \\
\hline
\end{tabular}



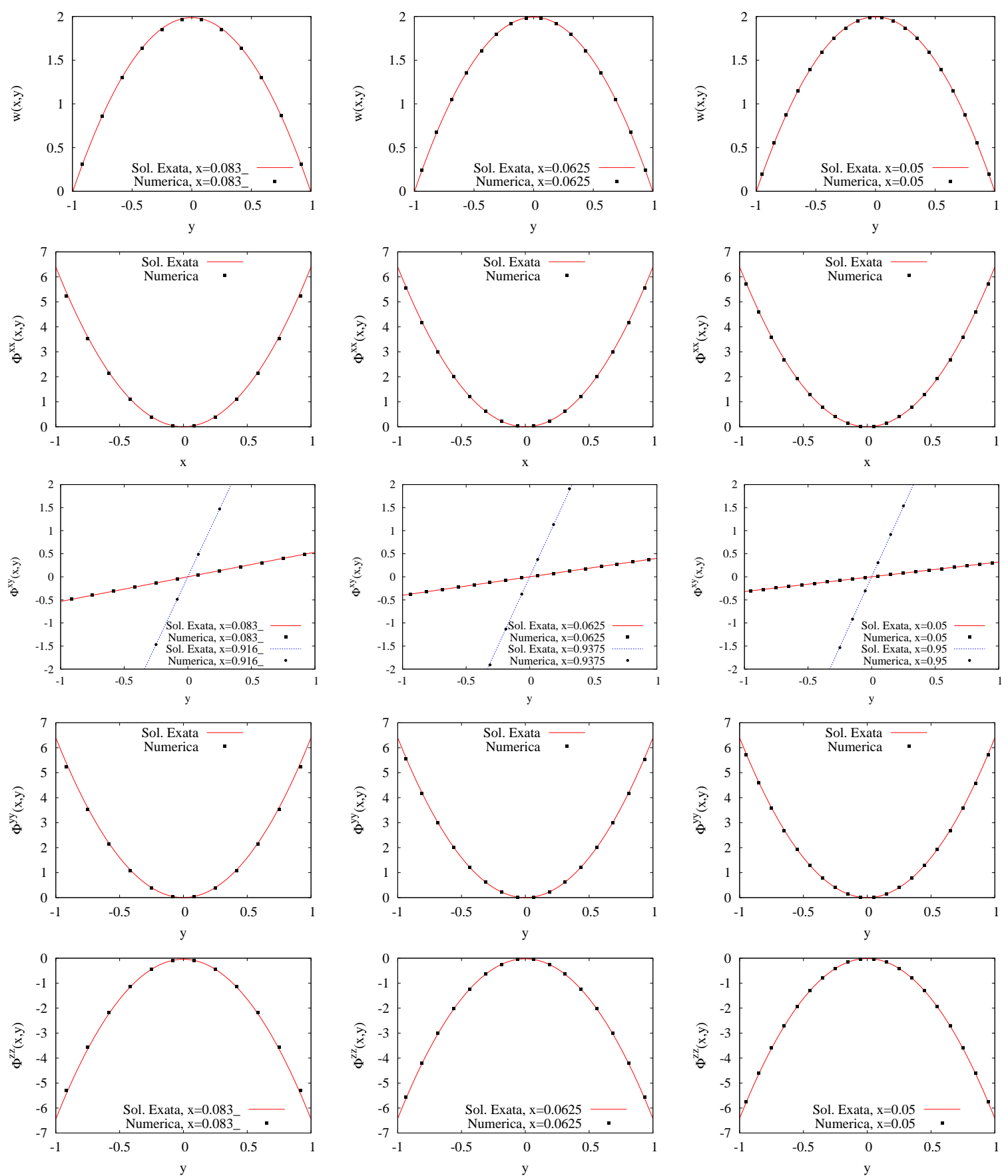

Figura 4.4: Comparação entre as soluções analíticas e as soluções numéricas para $w(x, y)$, $\Phi^{x x}, \Phi^{x y}, \Phi^{y y}, \Phi^{z z}$. Coluna da esquerda: malha M0. Coluna do meio: malha M1. Coluna da direita: malha M2. 


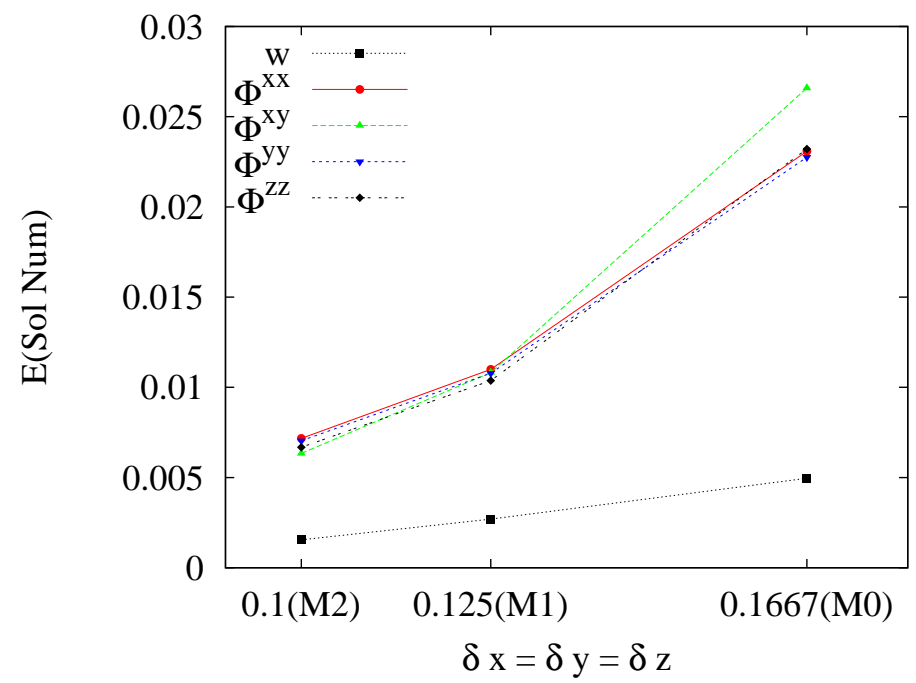

Figura 4.5: Decaimento dos erros com o refinamento da malha. 


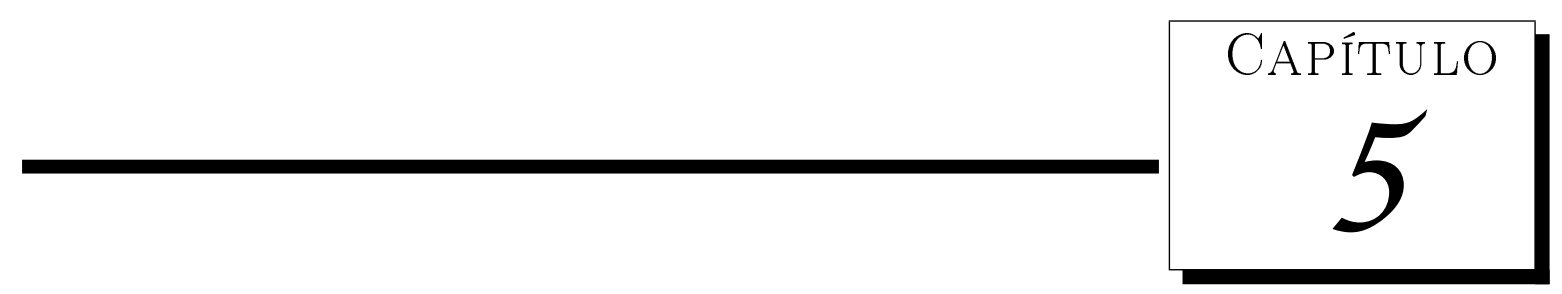

\section{Simulação numérica do inchamento do extrudado}

Nessa seção, apresentamos os resultados obtidos nas simulações do fenômeno conhecido como "inchamento do extrudado". Nesse problema, um jato escoa dentro de um tubo com diâmetro $D$ e na saída do tubo é expelido para a atmosfera, onde, sob certas condições, ocorre um aumento no diâmetro do jato que atinge um valor máximo $D_{\max }$. Sabe-se que, quanto maior for a elasticidade do fluido (representada pelo número de Deborah $D e$ ) maior será a taxa de inchamento $S_{r}=D_{\max } / D$ (ver Figura 5.1). Esse fenômeno está presente em diversas aplicações industriais, por exemplo, extrusão de polímeros e ligas metálicas, e tem sido objeto de estudo por muitos pesquisadores (por exemplo, [20],[34]-[45]).

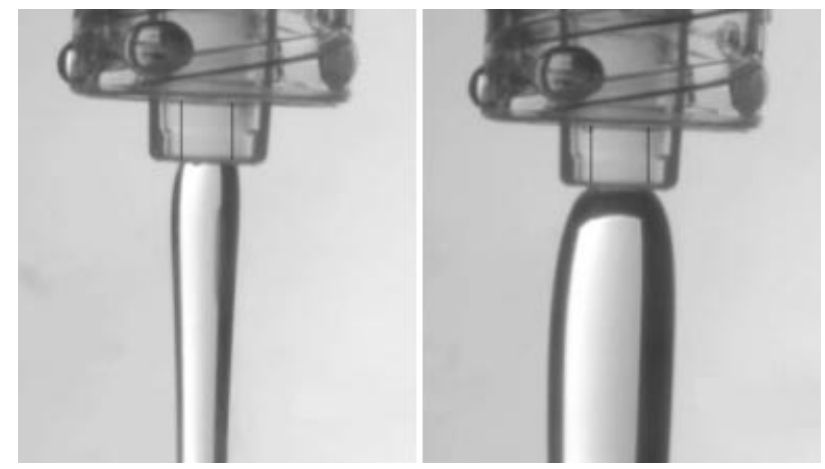

Figura 5.1: Extrudate swell.

Para demonstrar que o método numérico descrito neste trabalho é capaz de simular escoamentos viscoeláticos com superfície livre governados pela equação constitutiva do modelo SOF, simulamos o inchamento do extrudado de um jato modelado pela equação constitutiva SOF. Utilizando valores de referência (adimensionais), consideramos um tubo circular com raio $R=1$ e comprimento $L=5$.

Na entrada do tubo foi imposto escoamento de Poiseuille com velocidade $U=1$, conforme especificado na equação (4.1). As simulações começaram com o tubo vazio e o fluido foi injetado até atingir a saida do tubo onde deverá ocorrer o fenômeno inchamento do extrudado. Um contorno do tipo "outflow" foi colocado a uma distância de $2 L$ da saída do 
tubo. Uma malha com $32 \times 32 \times 120$ células $(\delta x=\delta y=\delta z=0.125)$ foi utilizada, semelhante a malha M1 utilizada na validação. Os dados utilizados nessas simulações são especificados na tabela 5.1. Essas simulações começaram em $t=0$ e foram efetuadas até $t=15$. A Figura 5.2 mostra a configuração do fluido para diferentes tempos. Os resultados obtidos em $t=15$ foram utilizados para calcular a taxa de inchamento obtida para cada $D e$, os quais são mostrados na tabela 5.1. Como podemos observar na tabela 5.1, as taxas obtidas foram $S_{r}=1.0734$ para $D e=0.15, S_{r}=1.0800$ para $D e=0.2$ e $S_{r}=1.2260$ para $D e=0.3$. Esses resultados são coerentes, porque quanto maior for o número de Deborah maior será a elasticidade do fluido e ,portanto, maior será a taxa de inchamento. Para verificar se o inchamento do extrudado é influenciado quando a malha é refinada, foi realizada uma simulação para $R e=1, D e=0.3$ e $\kappa=0.3$ usando uma malha (semelhante a M2) $40 \times 40 \times 150$ (ver Figura 5.3) e obteve-se uma taxa de inchamento $S_{r}=1.2655$. Esse resultado mostra que a malha utilizada pode interferir no inchamento do extrudado.

Tabela 5.1: Simulação numérica do inchamento do extrudado: taxas de inchamento obtidas.

\begin{tabular}{|c|c|c|c|c|}
\hline$R e$ & $D e$ & $\kappa$ & $S_{r}$ & Proporção \\
\hline \hline 1.0 & 0.15 & 0.075 & 1.0734 & $D e=2 \kappa$ \\
\hline 1.0 & 0.20 & 0.10 & 1.0800 & $D e=2 \kappa$ \\
\hline 1.0 & 0.30 & 0.30 & 1.2260 & $D e=\kappa$ \\
\hline
\end{tabular}



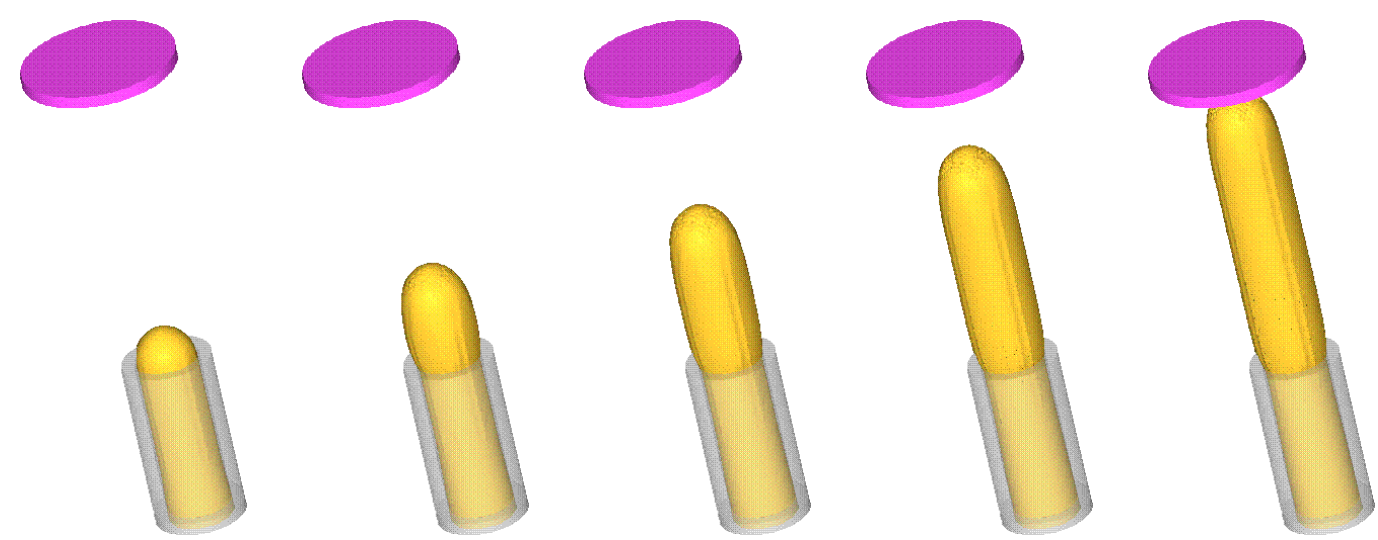

$$
D e=0.15, \kappa=0.075
$$
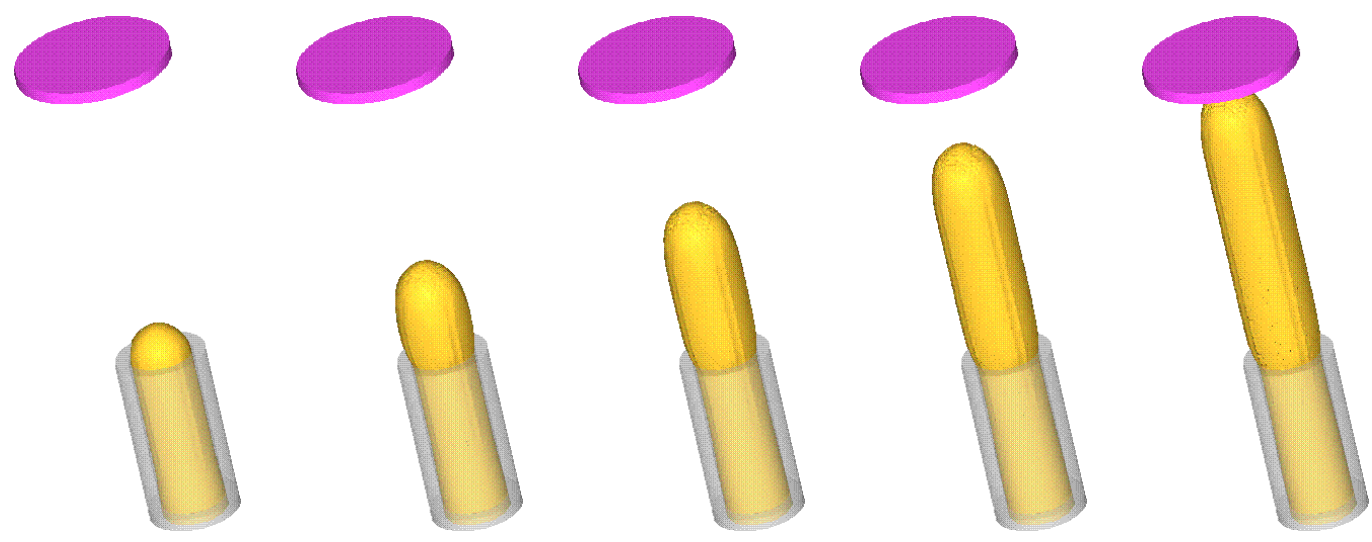

$$
D e=0.20, \kappa=0.10
$$
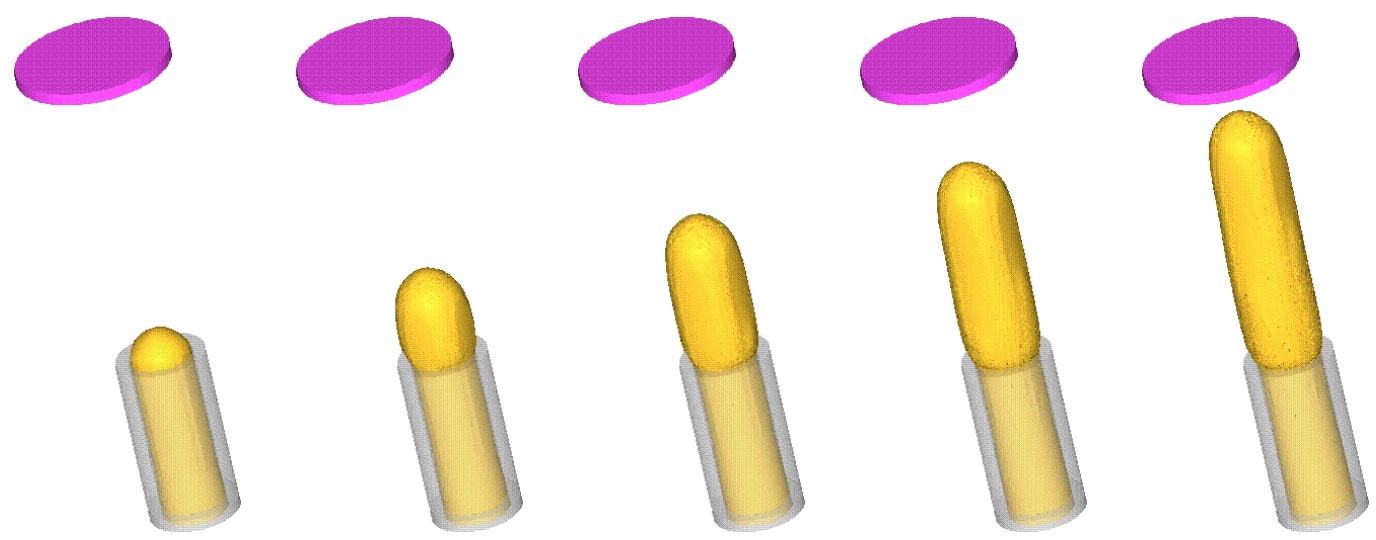

$$
D e=0.30, \kappa=0.30
$$

Figura 5.2: Simulação numérica do inchamento do extrudado para vários números $D e$ e $\kappa$ na malha intermediária (M1). Configuração do fluido nos tempos $t=5,7.5,10,12.5,15$. 

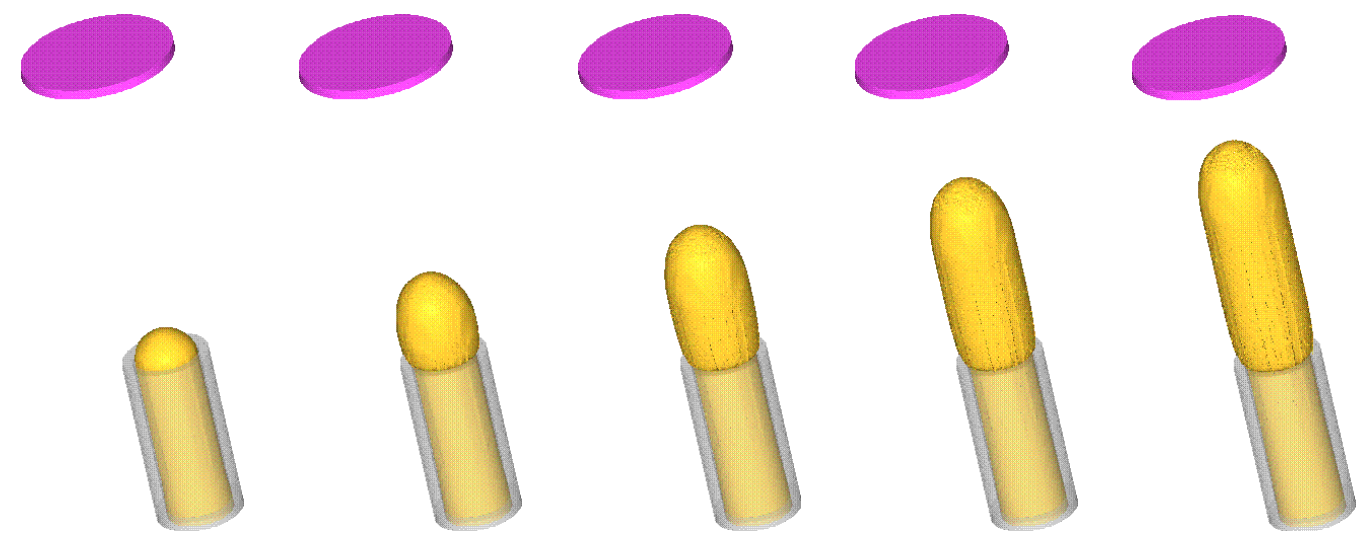

$$
D e=0.3, \kappa=0.3
$$

Figura 5.3: Simulação numérica do inchamento do extrudado na malha mais refinada (M2). Configuração do fluido nos tempos $t=5,7.5,10,12.5,15$. 


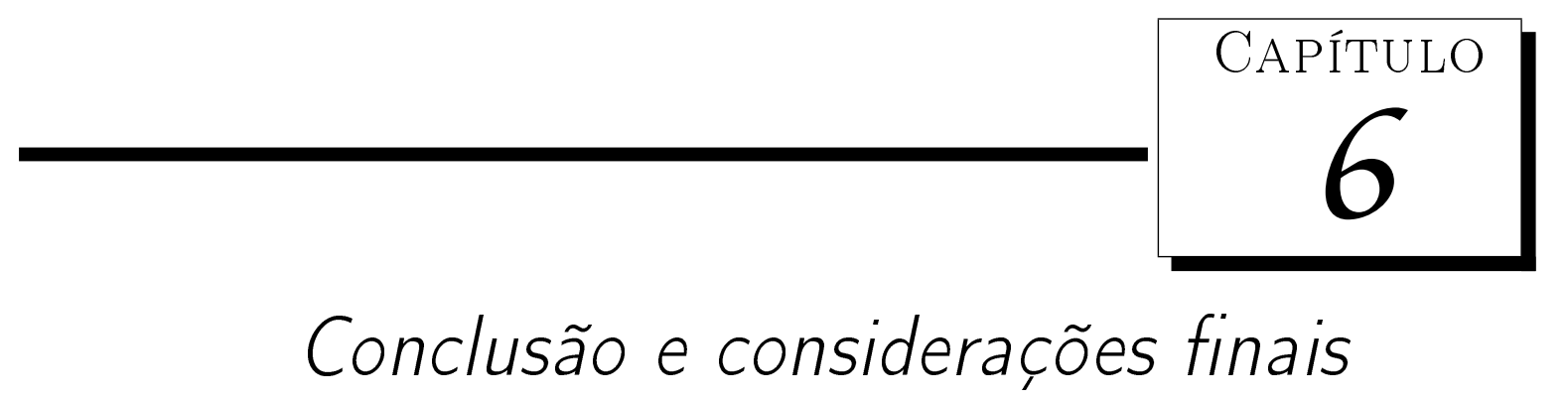

Esse trabalho apresentou os desenvolvimentos realizados para a obtenção de um método numérico para simular escoamentos incompressíveis com superfícies livres governados pela equação constitutiva Fluido de Segunda Orderm. As equações governantes foram derivadas em detalhes, bem como a adimensionalização empregada. As condições de contorno para a velocidade e as condições na superfície livre também foram descritas de maneira minuciosa. A obtenção da solução das equações governantes no âmbito da técnica GENSMAC3D [19] foi discutida detalhadamente, bem como a aproximação das equações pelo método de diferenças finitas. O método numérico desenvolvido nesse trabalho foi uma extensão da técnica bidimensional apresentada por Tomé et al. [13] para escoamentos tridimensionais. A validação do método numérico apresentado foi realizada através da simulação do escoamento desenvolvido em um tubo e os resultados obtidos foram comparados com a solução analítica para a velocidade $w(x, y)$ e o tensor não-Newtoniano $\boldsymbol{\Phi}$. A convergência do método apresentado foi verificada por meio de um estudo de refinamento de malha. Os resultados numéricos obtidos mostraram boa concordância com as soluções analíticas. Para demonstrar que o método numérico simula escoamentos viscoelásticos governados pela equação constitutiva Fluidos de Segunda Ordem, o problema do inchamento do extrudado foi simulado para vários valores das constantes temporais $\lambda_{2}$ e $\lambda_{4}$. Resultados foram obtidos para números de Deborah $D e \leq 0.3$.

Para dar continuidade a esse trabalho, pretendemos implementar um método implícito para resolver as equações de conservação de quantidade de movimento, bem como tratar as condições de contorno na superfície livre utilizando uma técnica implícita. Em particular, pretendemos utilizar o método implícito apresentado por Oishi et al. [46]. 


\section{Referências Bibliográficas}

[1] GK Batchelor. An Introduction to Fluid Dynamics. Cambridge University Press, 2000.

[2] R.B. Bird, R.C. Armstrong, and O. Hassager. Dynamics of polymeric liquids. 1. Fluid mechanics. Wiley, 1987.

[3] M. A. Alves, P. J. Oliveira, and F. T. Pinho, A convergent and universally bounded interpolation schemes for the treatment of advection, Intern. J. Numer. Meth. Fluids, 41 (2003), pp. $47-75$.

[4] M. A. Alves, P. J. Oliveira, and F. T. Pinho, Benchmark solutions for the flow of Oldroyd-b and PTT fluids in planar contractions, J. Non-Newtonian Fluid Mech., 110 (2003), pp. 45-75.

[5] T.N. Phillips, and A.J. Williams, Viscoelastic flow through a planar contraction using a semi-Lagrangian finite volume method, J. Non-Newtonian Fluid Mech., 87 (1999), pp. 215-246.

[6] T. Sato and S.M. Richardson, Explicit numerical simulation of time-dependent viscoelastic flows problems by a finite element/finite volume method, J. Non-Newtonian Fluid Mech., 51 (1994), pp. 249-275.

[7] M.F. Tomé, B. Duffy and S. McKee, A finite difference technique for simulating Non-Newtonian free surface flows, J. Non-Newtonian Fluid Mech., 62 (1996), pp. 9-34.

[8] S.-C. Xue, N. Phan-Thien and R.I. Tanner, Three dimensional numerical simulations of viscoelastic flows through planar contractions, J. Non-Newtonian Fluid Mech., $\mathbf{7 4}$ (1998), pp. 195-245.

[9] A. Varonos and G. Bergeles, Development and assessment of a variable-order non-oscillatory scheme for convection term discretization, Intern. J. Numer. Meth. Fluids, 26 (1998), pp. 1-16.

[10] J.Y. Yoo and Y. Na, A numerical study of the planar contraction flow of a viscoelastic fluid using the SIMPLER algorithm, J. Non-Newtonian Fluid Mech., 30 (1991), pp. 89-106.

[11] M.F. Tomé, S. McKee, GENSMAC: A computational marker-and-cell method for free surface flows in general domains, J. Comput. Phys., 110 (1994), pp.171-186. 
[12] M.F. Tomé, L. Grossi, A. Castelo, J.A. Cuminato, N. Mangiavacchi, V.G. Ferreira, F.S. de Sousa, S. McKee, A numerical method for solving three-dimensional generalized Newtonian free surface flows, J. Non-Newtonian Fluid Mech, 123 (2004), pp. 83-103.

[13] M. F. Tomé, J. L. Doricio, A. Castelo, J. A. Cuminato, S. McKee, Solving viscoelastic free surface flows of a second-order-fluid using a marker-and-cell approach, Intern. J. Numer. Meth. Fluids, 53 (2007), pp. 599-627.

[14] G. S. de Paulo, M. F. Tomé, S. McKee, A marker-and-cell approach to viscoelastic free surface flows using the PTT model, J. Non-Newtonian Fluid Mech, 147 (2007), pp. 149-174.

[15] E. Brasseur, M.M. Fyrillas, G.C. Georgiou, M.J. Crochet, The time-dependent extrudate-swell problem of an Oldroyd-B fluid with slip along the wall, J. Rheology. 42 (1998), pp. 549-566.

[16] E.O.A. Carew, P. Townsend, M.F. Webster, A Taylor-Petrov-Galerkin algorithm for viscoelastic flow, J. Non-Newtonian Fluid Mech. 50 (1993), pp. 253-287.

[17] A. Castelo, M. F. Tomé, C. N. L. Cézar, S. McKee, and J. A. Cuminato. Freeflow: an integrated simulation system for three-dimensional free surface flows. Computing and Visualization in Science, 2 (2000), pp. 199-210, 2000.

[18] A. B. Costacurta. Estratégias "upwind" e modelagem k-epsilon para simulação numérica de escoamentos com superfícies livres em altos números de Reynolds. Dissertação de Mestrado, ICMC/USP, 2005.

[19] M. F. Tomé, A. Castelo, J. A. Cuminato, N. Mangiavacchi, and S. McKee. Gensmac3D: A numerical method for solving unsteady three-dimensional free surface flows. Intern. J. Numer. Meth. Fluids, 37 (2001), pp. 747-796.

[20] M.F. Tomé, N. Mangiavacchi, J.A. Cuminato, A. Castelo, S. McKee, A finite difference technique for simulating unsteady viscoelastic free surface flows, J. Non-Newtonian Fluid Mech., 106 (2002), pp. 61-106.

[21] J.M. Marchal, M.J. Crochet, A new mixed finite element for calculating viscoelastic flow, J. Non-Newtonian Fluid Mech., 26 (1987), pp. 77-114.

[22] G. Mompean, M. Deville, Unsteady finite volume of Oldroyd-B fluid through a three-dimensional planar contraction, J. Non-Newtonian Fluid Mech., 72 (1997), pp. 253-279.

[23] M.J. Crochet and R. Keunings, Die swell of a Maxwell fluid - numerical prediction, J. Non-Newtonian Fluid Mech., 7 (1980), pp. 199-212.

[24] P.Correia, N. Arada e A. Sequeira, Analysis and finite element simulations of a second-order fluid model in a bounded domain, Numerical Methods for Partial Differential Equations, v23 (2007), Issue 6, pp. 1468-1500.

[25] Hayat, T., Khan, M., Siddiqui, A.M. and Asghar, S., Transient flows of a second grade fluid., Int. J. Non-Linear Mech., v39 (2002), pp. 1621-1633. 
[26] D. D. Joseph and J. Feng, A note on the forces that move particles in a second-order fluid, Non-Newtonian Fluid Mech, 64 (1996), pp. 299.

[27] D. D. Joseph, Bernoulli equation and the competition of elastic and inertial pressure in the potential flow of a second-order fluid, J. Non-Newtonian Fluid Mech, 42 (1991), pp. 358.

[28] J. E. Dunn and R. L. Fosdick, Thermodynamics, stability and boundedness of fluids of complexity 2 and fluids of second grade, Arch Rational Mech Anal, 56 (1974), pp. 191-252.

[29] J. E. Dunn and K. R. Rajagopal, Fluids of differential type: Critical review and thermodynamical analysis, Int J Engn Sci, 33 (1995), pp. 689-729.

[30] Fidap Manuals, Fluid Dynamics International, Evanstron, IL, 1995.

[31] L. Gast and W. Ellingson, Die swell measurements of second order fluids: numerical experiments, Intern. J. Numer. Meth. Fluids, 29 (1999), pp. 1-18.

[32] F. Harlow and J.E. Welch, Numerical Calculation of time-dependent viscous incompressible flow of fluid with a free surface, Phys. Fluids, 8 (1965), pp. 2182-2189.

[33] X. Huang, N. Phan-Thien and R.I. Tanner, Viscoelastic flow between eccentric rotating cylinders: Unstructured control volume method, J. Non-Newtonian Fluid Mech., 64 (1996), pp. 71-92.

[34] M. F. Tomé, A. Castelo, V. G. Ferreira, S. McKee, A finite difference technique for solving the Oldroyd B model for 3d-unsteady free surface flows, J. Non-Newtonian Fluid Mech., 154 (2008), pp. 179-206.

[35] M. F. Tomé, L. Grossi, A. Castelo, J. A. Cuminato, S. McKee, e K. Walters. Die-swell, splashing drop and a numerical technique for solving the Oldroyd-B model for axisymmetric free surface flows. J. Non-Newtonian Fluid Mech., 141: 148-166, 2007.

[36] R. I. Tanner. A theory of die-swell. J. Polymer Science, 8: 2067-2078, 1970.

[37] M. E. Ryan e A. Dutta. A finite difference simulation of extrudate swell. In Proceedings of Second World Congress of Chemical Engineering, volume VI, pages 277-281, 1981.

[38] M. J. Crochet e R. Keunings. Finite element analysis of die-swell of a highly elastic fluid. J. Non-Newtonian Fluid Mech., 10: 339-356, 1982.

[39] R. Keunings. An algorithm for the simulation of transient viscoelastic flows with free surfaces. J. Comput. Phys., 62 (1986), pp. 199-220.

[40] R. Keunings e D. Bousfield. Analysis of surface tension driven leveling in viscoelastic films. J. Non-Newtonian Fluid Mech., 22 (1987), pp. 219-233, .

[41] J. Y. Yoo e D. D. Joseph. Hyperbolicity and change of type in the flow of viscoelastic fluids through channels. J. Non-Newtonian Fluid Mech., 19 (1985), pp. 15-41.

[42] D. D. Joseph, J. E. Matta, e K. Chen. Delayed die swell. J. Non-Newtonian Fluid Mech., 24 (1987), pp. 31-65. 
[43] D. D. Joseph e C. Christodoulou. Independent confirmation that delayed die swell is a hyperbolic transition. J. Non-Newtonian Fluid Mech., 48 (1993), pp. 225-235.

[44] M. Cloitre, T. Hall, C. Mata, e D. D. Joseph. Delayed-die swell and sedimentation of elongated particles in wormlike micellar solutions. J. Non-Newtonian Fluid Mech., 79 (1998), pp. 157-171.

[45] R. I. Tanner. A theory of die-swell revisited. J. Non-Newtonian Fluid Mech., 129 (2005), pp. 85-87.

[46] C. M. Oishi, M. F. Tomé, J. A. Cuminato, and S. McKee, An implicit technique for solving 3D low Reynolds number moving free surface flows, J. Comput. Phys., 227 (2008), pp. 7446-7468. 
APÊNDICE

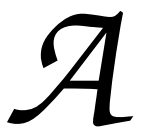

Discretização da função reológica $\mathbf{\Phi}$

A.1 Componente $\Phi^{x y}$ discretizada no centro da célula

$$
\begin{aligned}
\Phi_{i, j, k}^{x y}= & D e\left\{\left.\frac{\partial\left(u \mathrm{D}^{x y}\right)}{\partial x}\right|_{i, j, k}+\left.\frac{\partial\left(v \mathrm{D}^{x y}\right)}{\partial y}\right|_{i, j, k}+\left.\frac{\partial\left(w \mathrm{D}^{x y}\right)}{\partial z}\right|_{i, j, k}\right. \\
& -\left[\left.\left(\frac{\partial v}{\partial x} \mathrm{D}^{x x}\right)\right|_{i, j, k}+\left.\left[\left(\frac{\partial u}{\partial x}+\frac{\partial v}{\partial y}\right) \mathrm{D}^{x y}\right]\right|_{i, j, k}+\left.\left(\frac{\partial v}{\partial z} \mathrm{D}^{x z}\right)\right|_{i, j, k}+\left.\left(\frac{\partial u}{\partial y} \mathrm{D}^{y y}\right)\right|_{i, j, k}\right. \\
& \left.\left.+\left.\left(\frac{\partial u}{\partial z} \mathrm{D}^{y z}\right)\right|_{i, j, k}\right]\right\}+\kappa\left[\left.\left(\mathrm{D}^{x x} \mathrm{D}^{x y}\right)\right|_{i, j, k}+\left.\left(\mathrm{D}^{x y} \mathrm{D}^{y y}\right)\right|_{i, j, k}+\left.\left(\mathrm{D}^{x z} \mathrm{D}^{y z}\right)\right|_{i, j, k}\right]
\end{aligned}
$$

A.2 Componente $\Phi^{x z}$ discretizada no centro da célula

$$
\begin{aligned}
\Phi_{i, j, k}^{x z}= & D e\left\{\left.\frac{\partial\left(u \mathrm{D}^{x z}\right)}{\partial x}\right|_{i, j, k}+\left.\frac{\partial\left(v \mathrm{D}^{x z}\right)}{\partial y}\right|_{i, j, k}+\left.\frac{\partial\left(w \mathrm{D}^{x z}\right)}{\partial z}\right|_{i, j, k}\right. \\
& -\left[\left.\left(\frac{\partial w}{\partial x} \mathrm{D}^{x x}\right)\right|_{i, j, k}+\left.\left(\frac{\partial w}{\partial y} \mathrm{D}^{x y}\right)\right|_{i, j, k}+\left.\left[\left(\frac{\partial u}{\partial x}+\frac{\partial w}{\partial z}\right) \mathrm{D}^{x z}\right]\right|_{i, j, k}+\left.\left(\frac{\partial u}{\partial y} \mathrm{D}^{y z}\right)\right|_{i, j, k}\right. \\
& \left.\left.+\left.\left(\frac{\partial u}{\partial z} \mathrm{D}^{z z}\right)\right|_{i, j, k}\right]\right\}+\kappa\left[\left.\left(\mathrm{D}^{x x} \mathrm{D}^{x z}\right)\right|_{i, j, k}+\left.\left(\mathrm{D}^{x y} \mathrm{D}^{y z}\right)\right|_{i, j, k}+\left.\left(\mathrm{D}^{x z} \mathrm{D}^{z z}\right)\right|_{i, j, k}\right]
\end{aligned}
$$


A.3 Componente $\Phi^{y y}$ discretizada no centro da célula

$$
\begin{aligned}
\Phi_{i, j, k}^{y y}= & D e\left\{\left.\frac{\partial\left(u \mathrm{D}^{y y}\right)}{\partial x}\right|_{i, j, k}+\left.\frac{\partial\left(v \mathrm{D}^{y y}\right)}{\partial y}\right|_{i, j, k}+\left.\frac{\partial\left(w \mathrm{D}^{y y}\right)}{\partial z}\right|_{i, j, k}\right. \\
& \left.-2\left[\left.\left(\frac{\partial v}{\partial x} \mathrm{D}^{x y}\right)\right|_{i, j, k}+\left.\left(\frac{\partial v}{\partial y} \mathrm{D}^{y y}\right)\right|_{i, j, k}+\left.\left(\frac{\partial v}{\partial z} \mathrm{D}^{y z}\right)\right|_{i, j, k}\right]\right\} \\
& +\kappa\left[\left.\left(\mathrm{D}^{x y}\right)^{2}\right|_{i, j, k}+\left.\left(\mathrm{D}^{y y}\right)^{2}\right|_{i, j, k}+\left.\left(\mathrm{D}^{y z}\right)^{2}\right|_{i, j, k}\right]
\end{aligned}
$$

A.4 Componente $\Phi^{y z}$ discretizada no centro da célula

$$
\begin{aligned}
\Phi_{i, j, k}^{y z}= & D e\left\{\left.\frac{\partial\left(u \mathrm{D}^{y z}\right)}{\partial x}\right|_{i, j, k}+\left.\frac{\partial\left(v \mathrm{D}^{y z}\right)}{\partial y}\right|_{i, j, k}+\left.\frac{\partial\left(w \mathrm{D}^{y z}\right)}{\partial z}\right|_{i, j, k}\right. \\
& -\left[\left.\left(\frac{\partial w}{\partial x} \mathrm{D}^{x y}\right)\right|_{i, j, k}+\left.\left(\frac{\partial v}{\partial x} \mathrm{D}^{x z}\right)\right|_{i, j, k}+\left.\left(\frac{\partial w}{\partial y} \mathrm{D}^{y y}\right)\right|_{i, j, k}+\left.\left[\left(\frac{\partial v}{\partial y}+\frac{\partial w}{\partial z}\right) \mathrm{D}^{y z}\right]\right|_{i, j, k}\right. \\
& \left.\left.+\left.\left(\frac{\partial v}{\partial z} \mathrm{D}^{z z}\right)\right|_{i, j, k}\right]\right\}+\kappa\left[\left.\left(\mathrm{D}^{x y} \mathrm{D}^{x z}\right)\right|_{i, j, k}+\left.\left(\mathrm{D}^{y y} \mathrm{D}^{y z}\right)\right|_{i, j, k}+\left.\left(\mathrm{D}^{y z} \mathrm{D}^{z z}\right)\right|_{i, j, k}\right]
\end{aligned}
$$

A.5 Componente $\Phi^{z z}$ discretizada no centro da célula

$$
\begin{aligned}
\Phi_{i, j, k}^{z z}= & D e\left\{\left.\frac{\partial\left(u \mathrm{D}^{z z}\right)}{\partial x}\right|_{i, j, k}+\left.\frac{\partial\left(v \mathrm{D}^{z z}\right)}{\partial y}\right|_{i, j, k}+\left.\frac{\partial\left(w \mathrm{D}^{z z}\right)}{\partial z}\right|_{i, j, k}\right. \\
& \left.-2\left[\left.\left(\frac{\partial w}{\partial x} \mathrm{D}^{x z}\right)\right|_{i, j, k}+\left.\left(\frac{\partial w}{\partial y} \mathrm{D}^{y z}\right)\right|_{i, j, k}+\left.\left(\frac{\partial w}{\partial z} \mathrm{D}^{z z}\right)\right|_{i, j, k}\right]\right\} \\
& +\kappa\left[\left.\left(\mathrm{D}^{x z}\right)^{2}\right|_{i, j, k}+\left.\left(\mathrm{D}^{y z}\right)^{2}\right|_{i, j, k}+\left.\left(\mathrm{D}^{z z}\right)^{2}\right|_{i, j, k}\right]
\end{aligned}
$$


APÊNDICE

$\mathcal{B}$

Tratamento de superfícies planas consideradas no código FreeFlow3D

B.1 Superfície plana paralela a um eixo de coordenadas

B.1.1 Face $\left(i+\frac{1}{2}\right)$, onde $n=(1,0,0), m 1=(0,1,0)$ e $m 2=(0,0,1)$

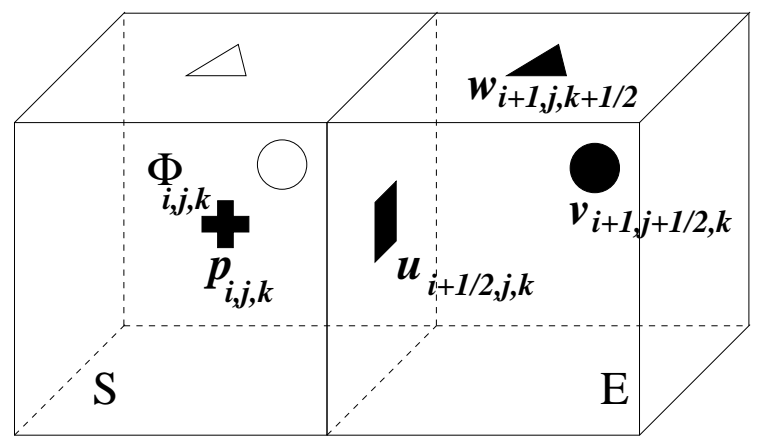

Figura B.1: Célula S com somente a face $\left(i+\frac{1}{2}\right)$ em contato com células E.

$$
\begin{gathered}
\tilde{p}=\frac{1}{R e}\left(2 \frac{\partial u}{\partial x}+\Phi^{x x}\right), \\
\left(\frac{\partial u}{\partial y}+\frac{\partial v}{\partial x}\right)=-\Phi^{x y}, \\
\left(\frac{\partial u}{\partial z}+\frac{\partial w}{\partial x}\right)=-\Phi^{x z} .
\end{gathered}
$$

Discretizando (B.2) no ponto $\left(i+\frac{1}{2}, j+\frac{1}{2}, k\right)$ temos

$$
\frac{u_{i+\frac{1}{2}, j+1, k}-u_{i+\frac{1}{2}, j, k}}{\delta y}+\frac{v_{i+1, j+\frac{1}{2}, k}-v_{i, j+\frac{1}{2}, k}}{\delta x}=-\Phi_{i+\frac{1}{2}, j+\frac{1}{2}, k}^{x y},
$$


e discretizando também a equação (B.3) no ponto $\left(i+\frac{1}{2}, j, k+\frac{1}{2}\right)$ obtém-se

$$
\frac{u_{i+\frac{1}{2}, j, k+1}-u_{i+\frac{1}{2}, j, k}}{\delta z}+\frac{w_{i+1, j, k+\frac{1}{2}}-w_{i, j, k+\frac{1}{2}}}{\delta x}=-\Phi_{i+\frac{1}{2}, j, k+\frac{1}{2}}^{x z}
$$

Agora, aplicando a equação da continuidade (1.75) no centro da célula S tem-se

$$
\frac{u_{i+\frac{1}{2}, j, k}-u_{i-\frac{1}{2}, j, k}}{\delta x}+\frac{v_{i, j+\frac{1}{2}, k}-v_{i, j-\frac{1}{2}, k}}{\delta y}+\frac{w_{i, j, k+\frac{1}{2}}-w_{i, j, k-\frac{1}{2}}}{\delta z}=0 .
$$

As equações (B.4)-(B.6) fornecem 3 equações para as incógnitas $u_{i+\frac{1}{2}, j, k}, v_{i+1, j+\frac{1}{2}, k}$ e $w_{i+1, j, k+\frac{1}{2}}$ que podem ser resolvidas facilmente. Calculamos $u_{i+\frac{1}{2}, j, k}$ pela equação da continuidade (B.6)

$$
u_{i+\frac{1}{2}, j, k}=u_{i-\frac{1}{2}, j, k}-\frac{\delta x}{\delta y}\left(v_{i, j+\frac{1}{2}, k}-v_{i, j-\frac{1}{2}, k}\right)-\frac{\delta x}{\delta z}\left(w_{i, j, k+\frac{1}{2}}-w_{i, j, k-\frac{1}{2}}\right)
$$

e $v_{i+1, j+\frac{1}{2}, k}$ e $w_{i+1, j, k+\frac{1}{2}}$ pelas equações (B.4) e (B.5), ou seja

$$
\begin{aligned}
& v_{i+1, j+\frac{1}{2}, k}=v_{i, j+\frac{1}{2}, k}-\frac{\delta x}{\delta y}\left(u_{i+\frac{1}{2}, j+1, k}-u_{i+\frac{1}{2}, j, k}\right)-\delta x \Phi_{i+\frac{1}{2}, j, k+\frac{1}{2}}^{x y} \\
& w_{i+1, j, k+\frac{1}{2}}=w_{i, j, k+\frac{1}{2}}-\frac{\delta x}{\delta z}\left(u_{i+\frac{1}{2}, j, k+1}-u_{i+\frac{1}{2}, j, k}\right)-\delta x \Phi_{i+\frac{1}{2}, j, k+\frac{1}{2}}^{x z}
\end{aligned}
$$

respectivamente. A pressão $\tilde{p}_{i, j, k}$ no centro da célula de superfície livre é então calculada usando (B.1) como

$$
\tilde{p}_{i, j, k}=\frac{1}{R e}\left(2 \frac{u_{i+\frac{1}{2}, j, k}-u_{i-\frac{1}{2}, j, k}}{\delta x}+\Phi_{i, j, k}^{x x}\right) .
$$

B.1.2 Face $\left(i-\frac{1}{2}\right)$, onde $n=(-1,0,0), m 1=(0,1,0)$ e $m 2=(0,0,1)$

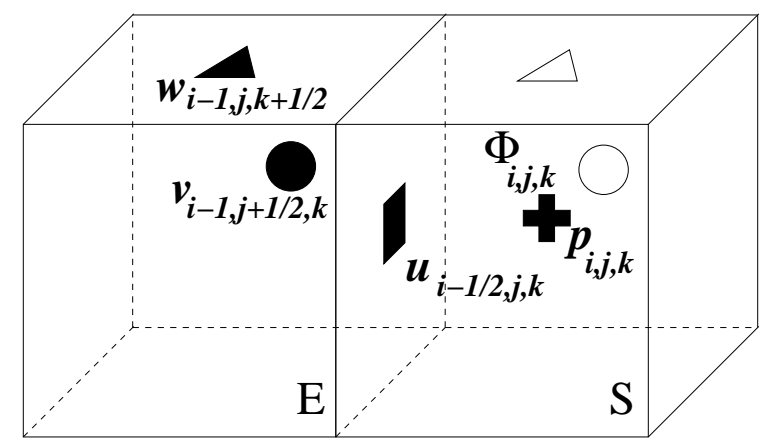

Figura B.2: Célula S com somente a face $\left(i-\frac{1}{2}\right)$ em contato com células E.

$$
\begin{gathered}
\tilde{p}=\frac{1}{R e}\left(2 \frac{\partial u}{\partial x}+\Phi^{x x}\right) \\
\left(\frac{\partial u}{\partial y}+\frac{\partial v}{\partial x}\right)=-\Phi^{x y}
\end{gathered}
$$




$$
\left(\frac{\partial u}{\partial z}+\frac{\partial w}{\partial x}\right)=-\Phi^{x z}
$$

As velocidades nas faces das células vazias e a pressão no centro da célula de superfície são calculadas pela discretização da equação da continuidade (1.75) no centro da célula $\mathrm{S}$ e as equações (B.12)-(B.13) nos pontos $\left(i-\frac{1}{2}, j+\frac{1}{2}, k\right)$ e $\left(i-\frac{1}{2}, j, k+\frac{1}{2}\right)$, respectivamente, como segue

$$
\begin{array}{r}
u_{i-\frac{1}{2}, j, k}=u_{i+\frac{1}{2}, j, k}+\frac{\delta x}{\delta y}\left(v_{i, j+\frac{1}{2}, k}-v_{i, j-\frac{1}{2}, k}\right)+\frac{\delta x}{\delta z}\left(w_{i, j, k+\frac{1}{2}}-w_{i, j, k-\frac{1}{2}}\right) \\
v_{i-1, j+\frac{1}{2}, k}=v_{i, j+\frac{1}{2}, k}+\frac{\delta x}{\delta y}\left(u_{i-\frac{1}{2}, j+1, k}-u_{i-\frac{1}{2}, j, k}\right)+\delta x \Phi_{i-\frac{1}{2}, j+\frac{1}{2}, k}^{x y} \\
w_{i-1, j, k+\frac{1}{2}}=w_{i, j, k+\frac{1}{2}}+\frac{\delta x}{\delta z}\left(u_{i-\frac{1}{2}, j, k+1}-u_{i-\frac{1}{2}, j, k}\right)+\delta x \Phi_{i-\frac{1}{2}, j, k+\frac{1}{2}}^{x z}
\end{array}
$$

A pressão $\tilde{p}_{i, j, k}$ é calculada por

$$
\tilde{p}_{i, j, k}=\frac{1}{R e}\left(2 \frac{u_{i+\frac{1}{2}, j, k}-u_{i-\frac{1}{2}, j, k}}{\delta x}+\Phi_{i, j, k}^{x x}\right) .
$$

B.1.3 Face $\left(j+\frac{1}{2}\right)$, onde $n=(0,1,0), m 1=(1,0,0)$ e $m 2=(0,0,1)$

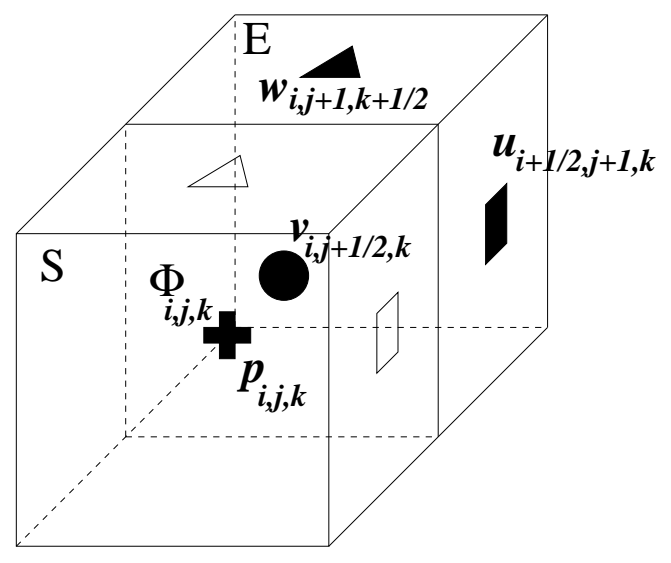

Figura B.3: Célula S com somente a face $\left(j+\frac{1}{2}\right)$ em contato com células E.

$$
\begin{gathered}
\tilde{p}=\frac{1}{R e}\left(2 \frac{\partial v}{\partial y}+\Phi^{y y}\right) \\
\left(\frac{\partial u}{\partial y}+\frac{\partial v}{\partial x}\right)=-\Phi^{x y} \\
\left(\frac{\partial v}{\partial z}+\frac{\partial w}{\partial y}\right)=-\Phi^{y z}
\end{gathered}
$$

De maneira análoga, obtemos

$$
v_{i, j+\frac{1}{2}, k}=v_{i, j-\frac{1}{2}, k}-\frac{\delta y}{\delta x}\left(u_{i+\frac{1}{2}, j, k}-u_{i-\frac{1}{2}, j, k}\right)-\frac{\delta y}{\delta z}\left(w_{i, j, k+\frac{1}{2}}-w_{i, j, k-\frac{1}{2}}\right),
$$




$$
\begin{gathered}
u_{i+\frac{1}{2}, j+1, k}=u_{i+\frac{1}{2}, j, k}-\frac{\delta y}{\delta x}\left(v_{i+1, j+\frac{1}{2}, k}-v_{i, j+\frac{1}{2}, k}\right)-\delta y \Phi_{i+\frac{1}{2}, j+\frac{1}{2}, k}^{x y}, \\
w_{i, j+1, k+\frac{1}{2}}=w_{i, j, k+\frac{1}{2}}-\frac{\delta y}{\delta z}\left(v_{i, j+\frac{1}{2}, k+1}-v_{i, j+\frac{1}{2}, k}\right)-\delta y \Phi_{i, j+\frac{1}{2}, k+\frac{1}{2}}^{y z} .
\end{gathered}
$$

A pressão $\tilde{p}_{i, j, k}$ é calculada por

$$
\tilde{p}_{i, j, k}=\frac{1}{R e}\left(2 \frac{v_{i, j+\frac{1}{2}, k}-v_{i, j-\frac{1}{2}, k}}{\delta y}+\Phi_{i, j, k}^{y y}\right) .
$$

B.1.4 Face $\left(j-\frac{1}{2}\right)$, onde $n=(0,-1,0), m 1=(1,0,0)$ e $m 2=(0,0,1)$

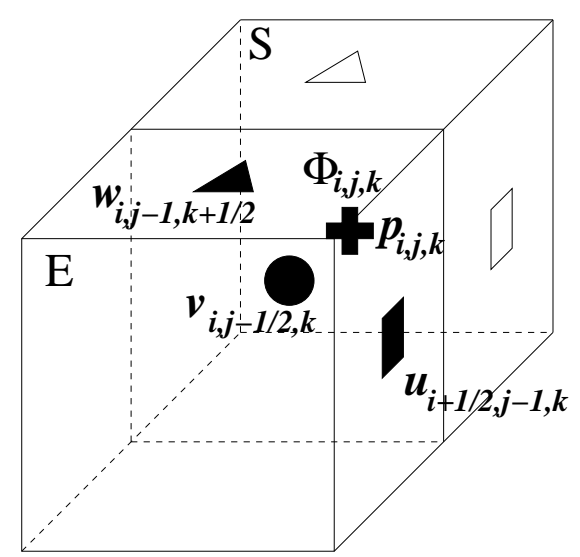

Figura B.4: Célula S com somente a face $\left(j-\frac{1}{2}\right)$ em contato com células E.

$$
\begin{gathered}
\tilde{p}=\frac{1}{R e}\left(2 \frac{\partial v}{\partial y}+\Phi^{y y}\right), \\
\left(\frac{\partial u}{\partial y}+\frac{\partial v}{\partial x}\right)=-\Phi^{x y}, \\
\left(\frac{\partial v}{\partial z}+\frac{\partial w}{\partial y}\right)=-\Phi^{y z}
\end{gathered}
$$

Discretizando a equação da continuidade e (B.25)-(B.27), obtemos

$$
\begin{array}{r}
v_{i, j-\frac{1}{2}, k}=v_{i, j+\frac{1}{2}, k}+\frac{\delta y}{\delta x}\left(u_{i+\frac{1}{2}, j, k}-u_{i-\frac{1}{2}, j, k}\right)+\frac{\delta y}{\delta z}\left(w_{i, j, k+\frac{1}{2}}-w_{i, j, k-\frac{1}{2}}\right) \\
u_{i+\frac{1}{2}, j-1, k}=u_{i+\frac{1}{2}, j, k}+\frac{\delta y}{\delta x}\left(v_{i+1, j-\frac{1}{2}, k}-v_{i, j-\frac{1}{2}, k}\right)+\delta y \Phi_{i+\frac{1}{2}, j-\frac{1}{2}, k}^{x y} \\
w_{i, j-1, k+\frac{1}{2}}=w_{i, j, k+\frac{1}{2}}+\frac{\delta y}{\delta z}\left(v_{i, j-\frac{1}{2}, k+1}-v_{i, j-\frac{1}{2}, k}\right)+\delta y \Phi_{i, j-\frac{1}{2}, k+\frac{1}{2}}^{y z}
\end{array}
$$

A pressão $\tilde{p}_{i, j, k}$ é calculada por

$$
\tilde{p}_{i, j, k}=\frac{1}{R e}\left(2 \frac{v_{i, j+\frac{1}{2}, k}-v_{i, j-\frac{1}{2}, k}}{\delta y}+\Phi_{i, j, k}^{y y}\right) .
$$


B.1.5 Face $\left(k-\frac{1}{2}\right)$, onde $n=(0,0,-1), m 1=(0,1,0)$ e $m 2=(1,0,0)$

$$
\begin{gathered}
\tilde{p}=\frac{1}{R e}\left(2 \frac{\partial w}{\partial z}+\Phi^{z z}\right) \\
\left(\frac{\partial v}{\partial z}+\frac{\partial w}{\partial y}\right)=-\Phi^{y z} \\
\left(\frac{\partial u}{\partial z}+\frac{\partial w}{\partial x}\right)=-\Phi^{x z}
\end{gathered}
$$

Discretizando a equação da continuidade e (B.32)-(B.34), obtemos

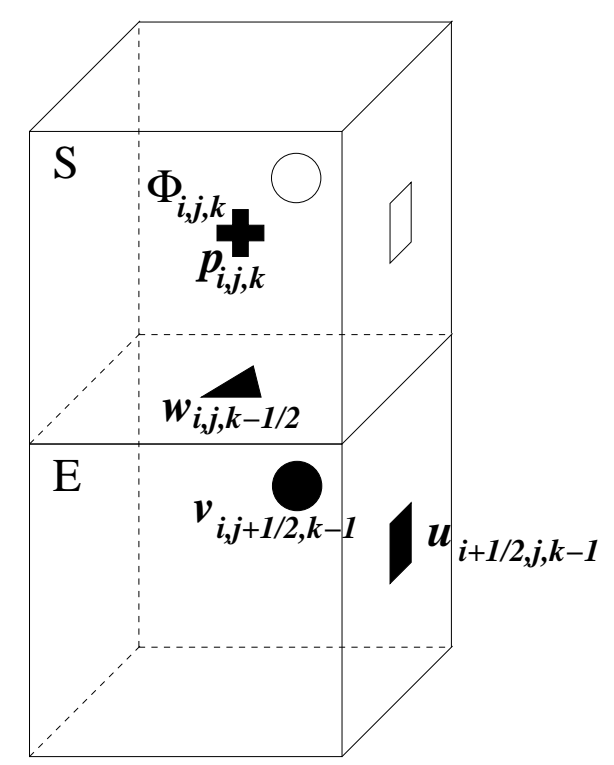

Figura B.5: Célula S com somente a face $\left(k-\frac{1}{2}\right)$ em contato com células E.

$$
\begin{gathered}
w_{i, j, k-\frac{1}{2}}=w_{i, j, k+\frac{1}{2}}+\frac{\delta z}{\delta x}\left(u_{i+\frac{1}{2}, j, k}-u_{i-\frac{1}{2}, j, k}\right)+\frac{\delta z}{\delta y}\left(v_{i, j+\frac{1}{2}, k}-v_{i, j-\frac{1}{2}, k}\right) \\
v_{i, j+\frac{1}{2}, k-1}=v_{i, j+\frac{1}{2}, k}+\frac{\delta z}{\delta y}\left(w_{i, j+1, k-\frac{1}{2}}-v_{i, j, k-\frac{1}{2}}\right)+\delta z \Phi_{i, j+\frac{1}{2}, k-\frac{1}{2}}^{y z} \\
u_{i+\frac{1}{2}, j, k-1}=u_{i+\frac{1}{2}, j, k}+\frac{\delta z}{\delta x}\left(w_{i+1, j, k-\frac{1}{2}}-w_{i, j, k-\frac{1}{2}}\right)+\delta z \Phi_{i+\frac{1}{2}, j, k-\frac{1}{2}}^{x z}
\end{gathered}
$$

A pressão $\tilde{p}_{i, j, k}$ é calculada por

$$
\tilde{p}_{i, j, k}=\frac{1}{R e}\left(2 \frac{w_{i, j, k+\frac{1}{2}}-w_{i, j, k-\frac{1}{2}}}{\delta z}+\Phi_{i, j, k}^{z z}\right) .
$$




\section{B.2 Superfície plana com inclinação de $45^{\circ}$}

B.2.1 Células $\mathbf{S}$ possuem apenas a face $\left(i+\frac{1}{2}\right)$ e $\left(j+\frac{1}{2}\right)$ em contato com células $\mathbf{E}$. Onde $n=\left(\frac{\sqrt{2}}{2}, \frac{\sqrt{2}}{2}, 0\right), m 1=\left(\frac{\sqrt{2}}{2},-\frac{\sqrt{2}}{2}, 0\right)$ e $m 2=$ $(0,0,1)$.

$$
\begin{gathered}
\tilde{p}=\frac{1}{R e}\left[\frac{\partial u}{\partial x}+\frac{\partial v}{\partial y}+\left(\frac{\partial u}{\partial y}+\frac{\partial v}{\partial x}\right)+\frac{1}{2}\left(\Phi^{x x}+\Phi^{y y}\right)+\Phi^{x y}\right] \\
\frac{\partial u}{\partial x}-\frac{\partial v}{\partial y}=-\frac{1}{2}\left(\Phi^{x x}-\Phi^{y y}\right)
\end{gathered}
$$

Discretizando (B.40)

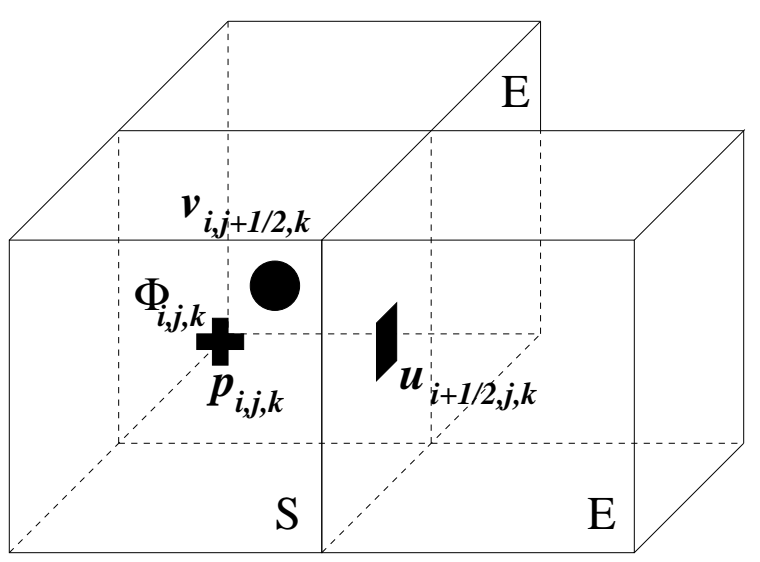

Figura B.6: Célula $\mathrm{S}$ com as faces $\left(i+\frac{1}{2}\right)$ e $\left(j+\frac{1}{2}\right)$ em contato com faces de células vazias.

$$
\frac{u_{i+\frac{1}{2}, j, k}-u_{i-\frac{1}{2}, j, k}}{\delta x}-\frac{v_{i, j+\frac{1}{2}, k}-v_{i, j-\frac{1}{2}, k}}{\delta y}=-\frac{1}{2}\left(\Phi_{i, j, k}^{x x}-\Phi_{i, j, k}^{y y}\right)
$$

e pela equação da continuidade (1.75) temos

$$
\frac{u_{i+\frac{1}{2}, j, k}-u_{i-\frac{1}{2}, j, k}}{\delta x}+\frac{v_{i, j+\frac{1}{2}, k}-v_{i, j-\frac{1}{2}, k}}{\delta y}=-\left(\frac{w_{i, j, k+\frac{1}{2}}-w_{i, j, k-\frac{1}{2}}}{\delta z}\right) .
$$

Temos como solução

$$
u_{i+\frac{1}{2}, j, k}=u_{i-\frac{1}{2}, j, k}-\frac{1}{2} \frac{\delta x}{\delta z}\left(w_{i, j, k+\frac{1}{2}}-w_{i, j, k-\frac{1}{2}}\right)-\frac{\delta x}{4}\left(\Phi_{i, j, k}^{x x}-\Phi_{i, j, k}^{y y}\right)
$$

e

$$
v_{i, j+\frac{1}{2}, k}=v_{i, j-\frac{1}{2}, k}-\frac{1}{2} \frac{\delta y}{\delta z}\left(w_{i, j, k+\frac{1}{2}}-w_{i, j, k-\frac{1}{2}}\right)+\frac{\delta y}{4}\left(\Phi_{i, j, k}^{x x}-\Phi_{i, j, k}^{y y}\right)
$$

Depois de calcudados $u_{i+\frac{1}{2}, j, k}$ e $v_{i, j+\frac{1}{2}, k}$, a pressão no centro da célula de superfície é obtida 
por

$$
\begin{aligned}
\tilde{p}= & \frac{1}{R e}\left[\frac{u_{i+\frac{1}{2}, j, k}-u_{i-\frac{1}{2}, j, k}}{\delta x}+\frac{v_{i, j+\frac{1}{2}, k}-v_{i, j-\frac{1}{2}, k}}{\delta y}\right. \\
& +\frac{1}{2}\left(\frac{u_{i+\frac{1}{2}, j, k}+u_{i-\frac{1}{2}, j, k}-u_{i+\frac{1}{2}, j-1, k}-u_{i-\frac{1}{2}, j-1, k}}{\delta y}\right. \\
& \left.+\frac{v_{i, j+\frac{1}{2}, k}+v_{i, j-\frac{1}{2}, k}-v_{i-1, j+\frac{1}{2}, k}-v_{i-1, j-\frac{1}{2}, k}}{\delta x}\right) \\
& \left.+\frac{1}{2}\left(\Phi_{i, j, k}^{x x}+\Phi_{i, j, k}^{y y}\right)+\Phi_{i, j, k}^{x y}\right] .
\end{aligned}
$$

B.2.2 Células $\mathbf{S}$ possuem apenas a face $\left(j+\frac{1}{2}\right)$ e $\left(k+\frac{1}{2}\right)$ em contato com células E. Onde $n=\left(0, \frac{\sqrt{2}}{2}, \frac{\sqrt{2}}{2}\right), m 1=\left(0, \frac{\sqrt{2}}{2},-\frac{\sqrt{2}}{2}\right)$ e $m 2=$ $(1,0,0)$.

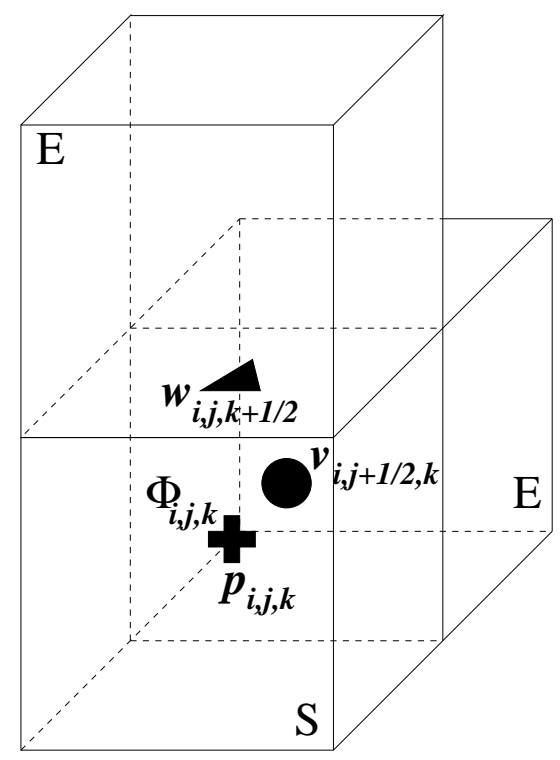

Figura B.7: Célula $\mathrm{S}$ com as faces $\left(j+\frac{1}{2}\right)$ e $\left(k+\frac{1}{2}\right)$ em contato com faces de células vazias.

$$
\begin{gathered}
\tilde{p}=\frac{1}{R e}\left[\frac{\partial v}{\partial y}+\frac{\partial w}{\partial z}+\left(\frac{\partial v}{\partial z}+\frac{\partial w}{\partial y}\right)+\frac{1}{2}\left(\Phi^{y y}+\Phi^{z z}\right)+\Phi^{y z}\right] \\
\frac{\partial v}{\partial y}-\frac{\partial w}{\partial z}=-\frac{1}{2}\left(\Phi^{y y}-\Phi^{z z}\right)
\end{gathered}
$$

Discretizando (B.47)

$$
\frac{v_{i, j+\frac{1}{2}, k}-v_{i, j-\frac{1}{2}, k}}{\delta y}-\frac{w_{i, j, k+\frac{1}{2}}-w_{i, j, k-\frac{1}{2}}}{\delta z}=-\frac{1}{2}\left(\Phi_{i, j, k}^{y y}-\Phi_{i, j, k}^{z z}\right)
$$

e pela equação da continuidade (1.75) temos

$$
\frac{v_{i, j+\frac{1}{2}, k}-v_{i, j-\frac{1}{2}, k}}{\delta y}+\frac{w_{i, j, k+\frac{1}{2}}-w_{i, j, k-\frac{1}{2}}}{\delta z}=-\left(\frac{u_{i+\frac{1}{2}, j, k}-u_{i-\frac{1}{2}, j, k}}{\delta x}\right) .
$$


Temos como solução

$$
v_{i, j+\frac{1}{2}, k}=v_{i, j-\frac{1}{2}, k}-\frac{1}{2} \frac{\delta y}{\delta x}\left(u_{i+\frac{1}{2}, j, k}-u_{i-\frac{1}{2}, j, k}\right)-\frac{\delta y}{4}\left(\Phi_{i, j, k}^{y y}-\Phi_{i, j, k}^{z z}\right)
$$

e

$$
w_{i, j, k+\frac{1}{2}}=w_{i, j, k-\frac{1}{2}}-\frac{1}{2} \frac{\delta z}{\delta x}\left(u_{i+\frac{1}{2}, j, k}-u_{i-\frac{1}{2}, j, k}\right)+\frac{\delta z}{4}\left(\Phi_{i, j, k}^{y y}-\Phi_{i, j, k}^{z z}\right)
$$

Depois de calcudados $v_{i, j+\frac{1}{2}, k}$ e $w_{i, j, k+\frac{1}{2}}$, a pressão no centro da célula de superfície é obtida por

$$
\begin{aligned}
\tilde{p}= & \frac{1}{R e}\left[\frac{v_{i, j+\frac{1}{2}, k}-v_{i, j-\frac{1}{2}, k}}{\delta y}+\frac{w_{i, j, k+\frac{1}{2}}-w_{i, j, k-\frac{1}{2}}}{\delta z}\right. \\
& +\frac{1}{2}\left(\frac{v_{i, j+\frac{1}{2}, k}+v_{i, j-\frac{1}{2}, k}-v_{i, j+\frac{1}{2}, k-1}-v_{i, j-\frac{1}{2}, k-1}}{\delta z}\right. \\
& \left.+\frac{w_{i, j, k+\frac{1}{2}}+w_{i, j, k-\frac{1}{2}}-w_{i, j-1, k+\frac{1}{2}}-w_{i, j-1, k-\frac{1}{2}}}{\delta y}\right) \\
& \left.+\frac{1}{2}\left(\Phi_{i, j, k}^{y y}+\Phi_{i, j, k}^{z z}\right)+\Phi_{i, j, k}^{y z}\right] .
\end{aligned}
$$

B.2.3 Células $\mathbf{S}$ possuem apenas a face $\left(i-\frac{1}{2}\right)$ e $\left(j+\frac{1}{2}\right)$ em contato com células E. Onde $n=\left(-\frac{\sqrt{2}}{2}, \frac{\sqrt{2}}{2}, 0\right), m 1=\left(\frac{\sqrt{2}}{2}, \frac{\sqrt{2}}{2}, 0\right)$ e $m 2=$ $(0,0,1)$.

$$
\begin{gathered}
\tilde{p}=\frac{1}{R e}\left[\frac{\partial u}{\partial x}+\frac{\partial v}{\partial y}-\left(\frac{\partial u}{\partial y}+\frac{\partial v}{\partial x}\right)+\frac{1}{2}\left(\Phi^{x x}+\Phi^{y y}\right)-\Phi^{x y}\right] \\
-\frac{\partial u}{\partial x}+\frac{\partial v}{\partial y}=-\frac{1}{2}\left(-\Phi^{x x}+\Phi^{y y}\right)
\end{gathered}
$$

Discretizando (B.54)

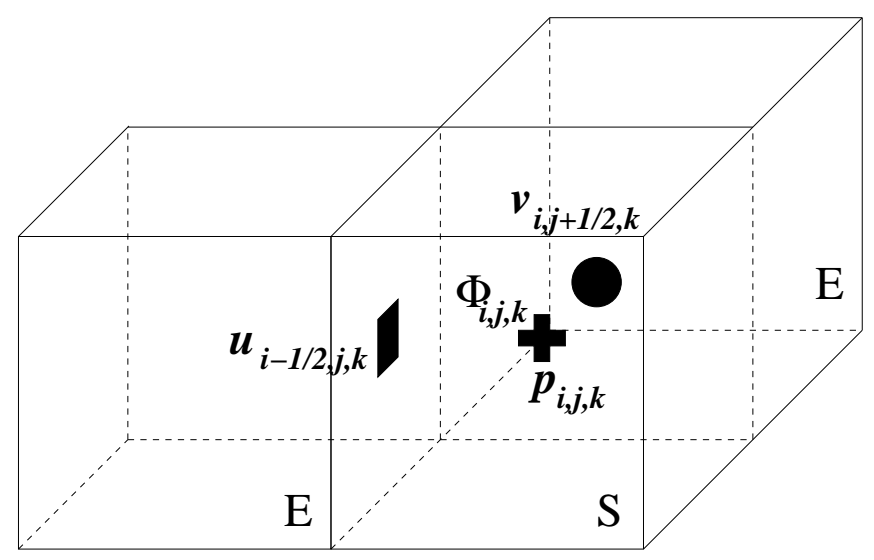

Figura B.8: Célula S com as faces $\left(i-\frac{1}{2}\right)$ e $\left(j+\frac{1}{2}\right)$ em contato com faces de células vazias.

$$
-\frac{u_{i+\frac{1}{2}, j, k}-u_{i-\frac{1}{2}, j, k}}{\delta x}+\frac{v_{i, j+\frac{1}{2}, k}-v_{i, j-\frac{1}{2}, k}}{\delta y}=-\frac{1}{2}\left(-\Phi_{i, j, k}^{x x}+\Phi_{i, j, k}^{y y}\right)
$$


e pela equação da continuidade (1.75) temos

$$
\frac{u_{i+\frac{1}{2}, j, k}-u_{i-\frac{1}{2}, j, k}}{\delta x}+\frac{v_{i, j+\frac{1}{2}, k}-v_{i, j-\frac{1}{2}, k}}{\delta y}=-\left(\frac{w_{i, j, k+\frac{1}{2}}-w_{i, j, k-\frac{1}{2}}}{\delta z}\right) .
$$

Temos como solução

$$
u_{i-\frac{1}{2}, j, k}=u_{i+\frac{1}{2}, j, k}+\frac{1}{2} \frac{\delta x}{\delta z}\left(w_{i, j, k+\frac{1}{2}}-w_{i, j, k-\frac{1}{2}}\right)-\frac{\delta x}{4}\left(-\Phi_{i, j, k}^{x x}+\Phi_{i, j, k}^{y y}\right)
$$

e

$$
v_{i, j+\frac{1}{2}, k}=v_{i, j-\frac{1}{2}, k}-\frac{1}{2} \frac{\delta y}{\delta z}\left(w_{i, j, k+\frac{1}{2}}-w_{i, j, k-\frac{1}{2}}\right)-\frac{\delta y}{4}\left(-\Phi_{i, j, k}^{x x}+\Phi_{i, j, k}^{y y}\right)
$$

Depois de calcudados $u_{i-\frac{1}{2}, j, k}$ e $v_{i, j+\frac{1}{2}, k}$, a pressão no centro da célula de superfície é obtida por

$$
\begin{aligned}
\tilde{p}= & \frac{1}{R e}\left[\frac{u_{i+\frac{1}{2}, j, k}-u_{i-\frac{1}{2}, j, k}}{\delta x}+\frac{v_{i, j+\frac{1}{2}, k}-v_{i, j-\frac{1}{2}, k}}{\delta y}\right. \\
& -\frac{1}{2}\left(\frac{u_{i+\frac{1}{2}, j, k}+u_{i-\frac{1}{2}, j, k}-u_{i+\frac{1}{2}, j-1, k}-u_{i-\frac{1}{2}, j-1, k}}{\delta y}\right. \\
& \left.+\frac{v_{i+1, j+\frac{1}{2}, k}+v_{i+1, j-\frac{1}{2}, k}-v_{i, j+\frac{1}{2}, k}-v_{i, j-\frac{1}{2}, k}}{\delta x}\right) \\
& \left.+\frac{1}{2}\left(\Phi_{i, j, k}^{x x}+\Phi_{i, j, k}^{y y}\right)-\Phi_{i, j, k}^{x y}\right] .
\end{aligned}
$$

B.2.4 Células $\mathbf{S}$ possuem apenas a face $\left(i-\frac{1}{2}\right)$ e $\left(k+\frac{1}{2}\right)$ em contato com células E. Onde $n=\left(-\frac{\sqrt{2}}{2}, 0, \frac{\sqrt{2}}{2}\right), m 1=\left(\frac{\sqrt{2}}{2}, 0, \frac{\sqrt{2}}{2}\right)$ e $m 2=$ $(0,1,0)$.

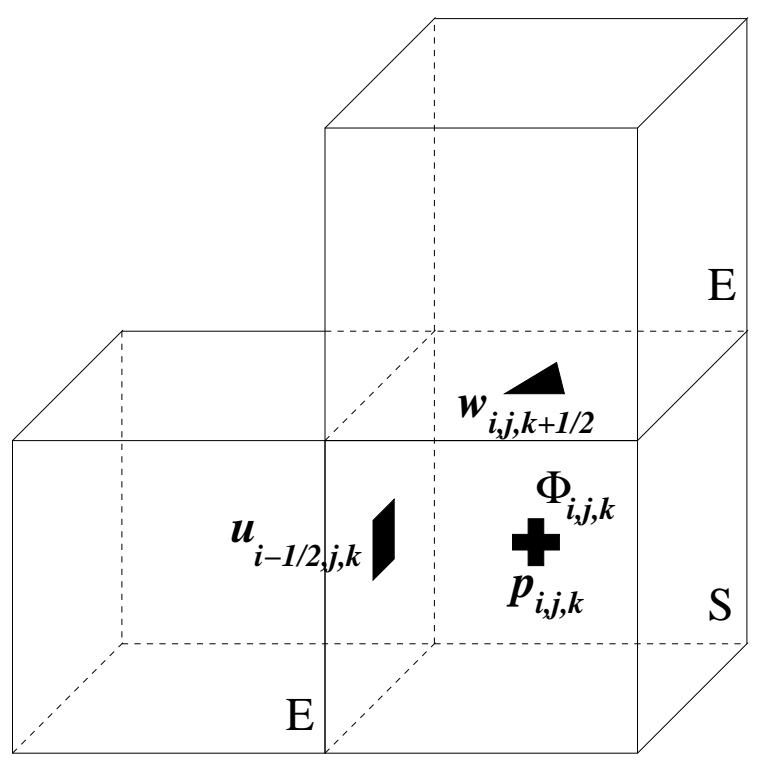

Figura B.9: Célula $S$ com as faces $\left(i-\frac{1}{2}\right)$ e $\left(k+\frac{1}{2}\right)$ em contato com faces de células vazias. 


$$
\begin{gathered}
\tilde{p}=\frac{1}{R e}\left[\frac{\partial u}{\partial x}+\frac{\partial w}{\partial z}-\left(\frac{\partial u}{\partial z}+\frac{\partial w}{\partial x}\right)+\frac{1}{2}\left(\Phi^{x x}+\Phi^{z z}\right)-\Phi^{x z}\right] \\
-\frac{\partial u}{\partial x}+\frac{\partial w}{\partial z}=-\frac{1}{2}\left(-\Phi^{x x}+\Phi^{z z}\right)
\end{gathered}
$$

Discretizando (B.61)

$$
-\frac{u_{i+\frac{1}{2}, j, k}-u_{i-\frac{1}{2}, j, k}}{\delta x}+\frac{w_{i, j, k+\frac{1}{2}}-w_{i, j, k-\frac{1}{2}}}{\delta z}=-\frac{1}{2}\left(-\Phi_{i, j, k}^{x x}+\Phi_{i, j, k}^{z z}\right)
$$

e pela equação da continuidade (1.75) temos

$$
\frac{u_{i+\frac{1}{2}, j, k}-u_{i-\frac{1}{2}, j, k}}{\delta x}+\frac{w_{i, j, k+\frac{1}{2}}-w_{i, j, k-\frac{1}{2}}}{\delta z}=-\left(\frac{v_{i, j+\frac{1}{2}, k}-v_{i, j-\frac{1}{2}, k}}{\delta y}\right) .
$$

Temos como solução

$$
u_{i-\frac{1}{2}, j, k}=u_{i+\frac{1}{2}, j, k}+\frac{1}{2} \frac{\delta x}{\delta y}\left(v_{i, j+\frac{1}{2}, k}-v_{i, j-\frac{1}{2}, k}\right)-\frac{\delta x}{4}\left(-\Phi_{i, j, k}^{x x}+\Phi_{i, j, k}^{z z}\right)
$$

e

$$
w_{i, j, k+\frac{1}{2}}=w_{i, j, k-\frac{1}{2}}-\frac{1}{2} \frac{\delta z}{\delta y}\left(v_{i, j+\frac{1}{2}, k}-v_{i, j-\frac{1}{2}, k}\right)-\frac{\delta z}{4}\left(-\Phi_{i, j, k}^{x x}+\Phi_{i, j, k}^{z z}\right)
$$

Depois de calcudados $u_{i-\frac{1}{2}, j, k}$ e $w_{i, j, k+\frac{1}{2}}$, a pressão no centro da célula de superfície é obtida por

$$
\begin{aligned}
\tilde{p}= & \frac{1}{R e}\left[\frac{u_{i+\frac{1}{2}, j, k}-u_{i-\frac{1}{2}, j, k}}{\delta x}+\frac{w_{i, j, k+\frac{1}{2}}-w_{i, j, k-\frac{1}{2}}}{\delta z}\right. \\
& -\frac{1}{2}\left(\frac{u_{i+\frac{1}{2}, j, k}+u_{i-\frac{1}{2}, j, k}-u_{i+\frac{1}{2}, j, k-1}-u_{i-\frac{1}{2}, j, k-1}}{\delta z}\right. \\
& \left.+\frac{w_{i+1, j, k+\frac{1}{2}}+w_{i+1, j, k-\frac{1}{2}}-w_{i, j, k+\frac{1}{2}}-w_{i, j, k-\frac{1}{2}}}{\delta x}\right) \\
& \left.+\frac{1}{2}\left(\Phi_{i, j, k}^{x x}+\Phi_{i, j, k}^{z z}\right)-\Phi_{i, j, k}^{x z}\right] .
\end{aligned}
$$

B.2.5 Células $\mathbf{S}$ possuem apenas a face $\left(i+\frac{1}{2}\right)$ e $\left(j-\frac{1}{2}\right)$ em contato com células E. Onde $n=\left(\frac{\sqrt{2}}{2},-\frac{\sqrt{2}}{2}, 0\right), m 1=\left(\frac{\sqrt{2}}{2}, \frac{\sqrt{2}}{2}, 0\right)$ e $m 2=$ $(0,0,1)$.

$$
\begin{gathered}
\tilde{p}=\frac{1}{R e}\left[\frac{\partial u}{\partial x}+\frac{\partial v}{\partial y}-\left(\frac{\partial u}{\partial y}+\frac{\partial v}{\partial x}\right)+\frac{1}{2}\left(\Phi^{x x}+\Phi^{y y}\right)-\Phi^{x y}\right] \\
\frac{\partial u}{\partial x}-\frac{\partial v}{\partial y}=-\frac{1}{2}\left(\Phi^{x x}-\Phi^{y y}\right)
\end{gathered}
$$

Discretizando (B.68)

$$
\frac{u_{i+\frac{1}{2}, j, k}-u_{i-\frac{1}{2}, j, k}}{\delta x}-\frac{v_{i, j+\frac{1}{2}, k}-v_{i, j-\frac{1}{2}, k}}{\delta y}=-\frac{1}{2}\left(\Phi_{i, j, k}^{x x}-\Phi_{i, j, k}^{y y}\right)
$$




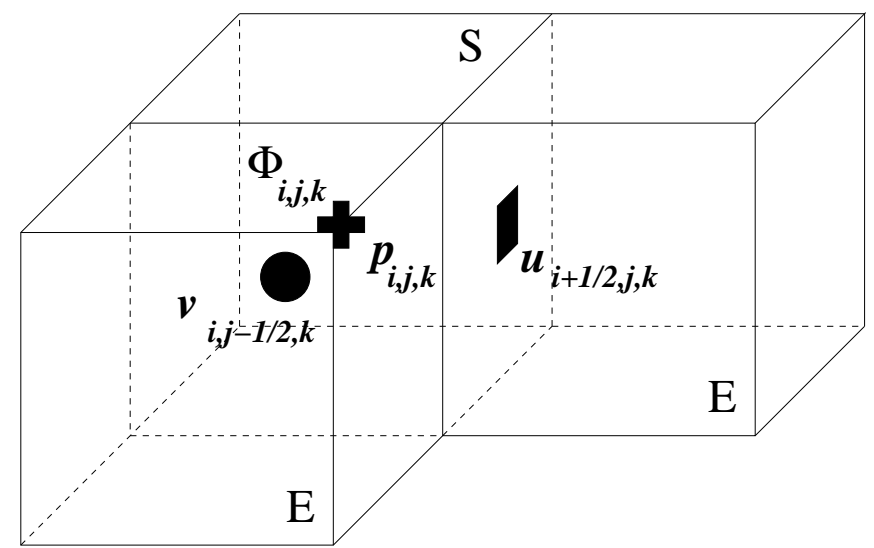

Figura B.10: Célula S com as faces $\left(i+\frac{1}{2}\right)$ e $\left(j-\frac{1}{2}\right)$ em contato com faces de células vazias. e pela equação da continuidade (1.75) temos

$$
\frac{u_{i+\frac{1}{2}, j, k}-u_{i-\frac{1}{2}, j, k}}{\delta x}+\frac{v_{i, j+\frac{1}{2}, k}-v_{i, j-\frac{1}{2}, k}}{\delta y}=-\left(\frac{w_{i, j, k+\frac{1}{2}}-w_{i, j, k-\frac{1}{2}}}{\delta z}\right) .
$$

Temos como solução

$$
u_{i+\frac{1}{2}, j, k}=u_{i-\frac{1}{2}, j, k}-\frac{1}{2} \frac{\delta x}{\delta z}\left(w_{i, j, k+\frac{1}{2}}-w_{i, j, k-\frac{1}{2}}\right)-\frac{\delta x}{4}\left(\Phi_{i, j, k}^{x x}-\Phi_{i, j, k}^{y y}\right)
$$

e

$$
v_{i, j-\frac{1}{2}, k}=v_{i, j+\frac{1}{2}, k}+\frac{1}{2} \frac{\delta y}{\delta z}\left(w_{i, j, k+\frac{1}{2}}-w_{i, j, k-\frac{1}{2}}\right)-\frac{\delta y}{4}\left(\Phi_{i, j, k}^{x x}-\Phi_{i, j, k}^{y y}\right)
$$

Depois de calcudados $u_{i+\frac{1}{2}, j, k}$ e $v_{i, j-\frac{1}{2}, k}$, a pressão no centro da célula de superfície é obtida por

$$
\begin{aligned}
\tilde{p}= & \frac{1}{R e}\left[\frac{u_{i+\frac{1}{2}, j, k}-u_{i-\frac{1}{2}, j, k}}{\delta x}+\frac{v_{i, j+\frac{1}{2}, k}-v_{i, j-\frac{1}{2}, k}}{\delta y}\right. \\
& -\frac{1}{2}\left(\frac{u_{i+\frac{1}{2}, j+1, k}+u_{i-\frac{1}{2}, j+1, k}-u_{i+\frac{1}{2}, j, k}-u_{i-\frac{1}{2}, j, k}}{\delta y}\right. \\
& \left.+\frac{v_{i, j+\frac{1}{2}, k}+v_{i, j-\frac{1}{2}, k}-v_{i-1, j+\frac{1}{2}, k}-v_{i-1, j-\frac{1}{2}, k}}{\delta x}\right) \\
& \left.+\frac{1}{2}\left(\Phi_{i, j, k}^{x x}+\Phi_{i, j, k}^{y y}\right)-\Phi_{i, j, k}^{x y}\right] .
\end{aligned}
$$

B.2.6 Células $S$ possuem apenas a face $\left(j-\frac{1}{2}\right)$ e $\left(k+\frac{1}{2}\right)$ em contato com células $\mathbf{E}$. Onde $n=\left(0,-\frac{\sqrt{2}}{2}, \frac{\sqrt{2}}{2}\right), m 1=\left(0, \frac{\sqrt{2}}{2}, \frac{\sqrt{2}}{2}\right)$ e $m 2=$ $(1,0,0)$.

$$
\begin{gathered}
\tilde{p}=\frac{1}{R e}\left[\frac{\partial v}{\partial y}+\frac{\partial w}{\partial z}-\left(\frac{\partial v}{\partial z}+\frac{\partial w}{\partial y}\right)+\frac{1}{2}\left(\Phi^{y y}+\Phi^{z z}\right)-\Phi^{y z}\right] \\
-\frac{\partial v}{\partial y}+\frac{\partial w}{\partial z}=-\frac{1}{2}\left(-\Phi^{y y}+\Phi^{z z}\right)
\end{gathered}
$$




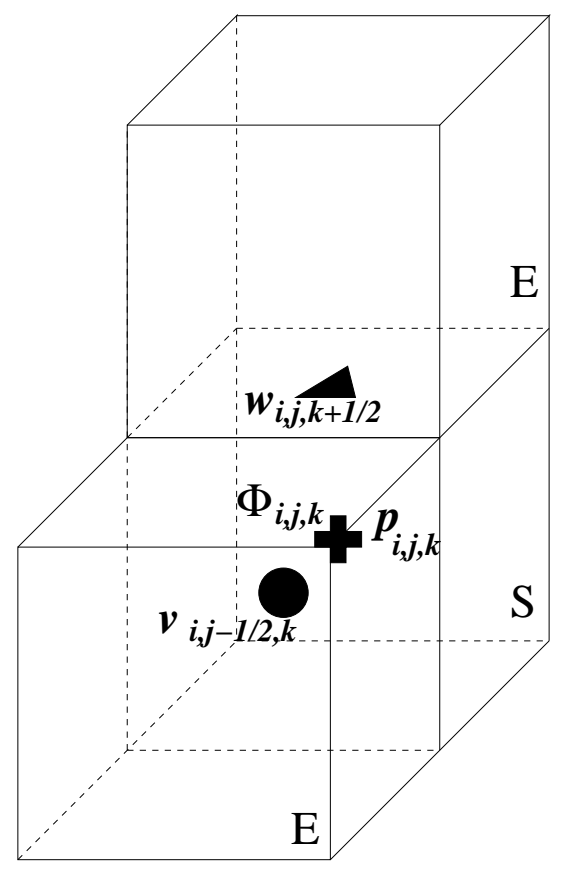

Figura B.11: Célula S com as faces $\left(j-\frac{1}{2}\right)$ e $\left(k+\frac{1}{2}\right)$ em contato com faces de células vazias.

Discretizando (B.75)

$$
-\frac{v_{i, j+\frac{1}{2}, k}-v_{i, j-\frac{1}{2}, k}}{\delta y}+\frac{w_{i, j, k+\frac{1}{2}}-w_{i, j, k-\frac{1}{2}}}{\delta z}=-\frac{1}{2}\left(-\Phi_{i, j, k}^{y y}+\Phi_{i, j, k}^{z z}\right)
$$

e pela equação da continuidade (1.75) temos

$$
\frac{v_{i, j+\frac{1}{2}, k}-v_{i, j-\frac{1}{2}, k}}{\delta y}+\frac{w_{i, j, k+\frac{1}{2}}-w_{i, j, k-\frac{1}{2}}}{\delta z}=-\left(\frac{u_{i+\frac{1}{2}, j, k}-u_{i-\frac{1}{2}, j, k}}{\delta x}\right) .
$$

Temos como solução

$$
v_{i, j-\frac{1}{2}, k}=v_{i, j+\frac{1}{2}, k}+\frac{1}{2} \frac{\delta y}{\delta x}\left(u_{i+\frac{1}{2}, j, k}-u_{i-\frac{1}{2}, j, k}\right)-\frac{\delta y}{4}\left(-\Phi_{i, j, k}^{y y}+\Phi_{i, j, k}^{z z}\right)
$$

e

$$
w_{i, j, k+\frac{1}{2}}=w_{i, j, k-\frac{1}{2}}-\frac{1}{2} \frac{\delta z}{\delta x}\left(u_{i+\frac{1}{2}, j, k}-u_{i-\frac{1}{2}, j, k}\right)-\frac{\delta z}{4}\left(-\Phi_{i, j, k}^{y y}+\Phi_{i, j, k}^{z z}\right)
$$

Depois de calcudados $v_{i, j-\frac{1}{2}, k}$ e $w_{i, j, k+\frac{1}{2}}$, a pressão no centro da célula de superfície é obtida por

$$
\begin{aligned}
\tilde{p}= & \frac{1}{R e}\left[\frac{v_{i, j+\frac{1}{2}, k}-v_{i, j-\frac{1}{2}, k}}{\delta y}+\frac{w_{i, j, k+\frac{1}{2}}-w_{i, j, k-\frac{1}{2}}}{\delta z}\right. \\
& -\frac{1}{2}\left(\frac{v_{i, j+\frac{1}{2}, k}+v_{i, j-\frac{1}{2}, k}-v_{i, j+\frac{1}{2}, k-1}-v_{i, j-\frac{1}{2}, k-1}}{\delta z}\right. \\
& \left.+\frac{w_{i, j+1, k+\frac{1}{2}}+w_{i, j+1, k-\frac{1}{2}}-w_{i, j, k+\frac{1}{2}}-w_{i, j, k-\frac{1}{2}}}{\delta y}\right) \\
& \left.+\frac{1}{2}\left(\Phi_{i, j, k}^{y y}+\Phi_{i, j, k}^{z z}\right)-\Phi_{i, j, k}^{y z}\right] .
\end{aligned}
$$




\section{B.2.7 Células $\mathbf{S}$ possuem apenas a face $\left(i-\frac{1}{2}\right)$ e $\left(j-\frac{1}{2}\right)$ em contato} com células $\mathrm{E}$. Onde $n=\left(-\frac{\sqrt{2}}{2},-\frac{\sqrt{2}}{2}, 0\right), m 1=\left(\frac{\sqrt{2}}{2},-\frac{\sqrt{2}}{2}, 0\right)$ e $m 2=(0,0,1)$.

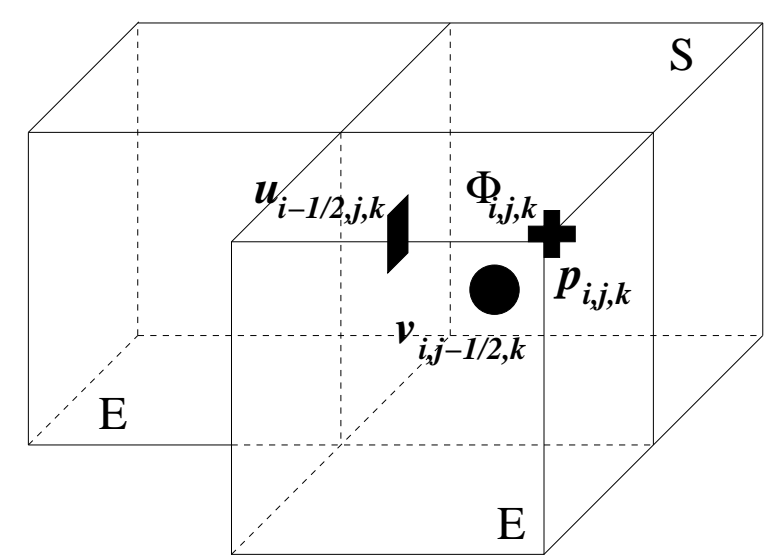

Figura B.12: Célula S com as faces $\left(i-\frac{1}{2}\right)$ e $\left(j-\frac{1}{2}\right)$ em contato com faces de células vazias.

$$
\begin{gathered}
\tilde{p}=\frac{1}{R e}\left[\frac{\partial u}{\partial x}+\frac{\partial v}{\partial y}+\left(\frac{\partial u}{\partial y}+\frac{\partial v}{\partial x}\right)+\frac{1}{2}\left(\Phi^{x x}+\Phi^{y y}\right)+\Phi^{x y}\right] \\
-\frac{\partial u}{\partial x}+\frac{\partial v}{\partial y}=-\frac{1}{2}\left(-\Phi^{x x}+\Phi^{y y}\right)
\end{gathered}
$$

Discretizando (B.82)

$$
-\frac{u_{i+\frac{1}{2}, j, k}-u_{i-\frac{1}{2}, j, k}}{\delta x}+\frac{v_{i, j+\frac{1}{2}, k}-v_{i, j-\frac{1}{2}, k}}{\delta y}=-\frac{1}{2}\left(-\Phi_{i, j, k}^{x x}+\Phi_{i, j, k}^{y y}\right)
$$

e pela equação da continuidade (1.75) temos

$$
\frac{u_{i+\frac{1}{2}, j, k}-u_{i-\frac{1}{2}, j, k}}{\delta x}+\frac{v_{i, j+\frac{1}{2}, k}-v_{i, j-\frac{1}{2}, k}}{\delta y}=-\left(\frac{w_{i, j, k+\frac{1}{2}}-w_{i, j, k-\frac{1}{2}}}{\delta z}\right) .
$$

Temos como solução

$$
u_{i-\frac{1}{2}, j, k}=u_{i+\frac{1}{2}, j, k}+\frac{1}{2} \frac{\delta x}{\delta z}\left(w_{i, j, k+\frac{1}{2}}-w_{i, j, k-\frac{1}{2}}\right)-\frac{\delta x}{4}\left(-\Phi_{i, j, k}^{x x}+\Phi_{i, j, k}^{y y}\right)
$$

e

$$
v_{i, j-\frac{1}{2}, k}=v_{i, j+\frac{1}{2}, k}+\frac{1}{2} \frac{\delta y}{\delta z}\left(w_{i, j, k+\frac{1}{2}}-w_{i, j, k-\frac{1}{2}}\right)+\frac{\delta y}{4}\left(-\Phi_{i, j, k}^{x x}+\Phi_{i, j, k}^{y y}\right)
$$

Depois de calcudados $u_{i-\frac{1}{2}, j, k}$ e $v_{i, j-\frac{1}{2}, k}$, a pressão no centro da célula de superfície é obtida 
por

$$
\begin{aligned}
\tilde{p}= & \frac{1}{R e}\left[\frac{u_{i+\frac{1}{2}, j, k}-u_{i-\frac{1}{2}, j, k}}{\delta x}+\frac{v_{i, j+\frac{1}{2}, k}-v_{i, j-\frac{1}{2}, k}}{\delta y}\right. \\
& +\frac{1}{2}\left(\frac{u_{i+\frac{1}{2}, j+1, k}+u_{i-\frac{1}{2}, j+1, k}-u_{i+\frac{1}{2}, j, k}-u_{i-\frac{1}{2}, j, k}}{\delta y}\right. \\
& \left.+\frac{v_{i+1, j+\frac{1}{2}, k}+v_{i+1, j-\frac{1}{2}, k}-v_{i, j+\frac{1}{2}, k}-v_{i, j-\frac{1}{2}, k}}{\delta x}\right) \\
& \left.+\frac{1}{2}\left(\Phi_{i, j, k}^{x x}+\Phi_{i, j, k}^{y y}\right)+\Phi_{i, j, k}^{x y}\right] .
\end{aligned}
$$

B.2.8 Células $\mathbf{S}$ possuem apenas a face $\left(i+\frac{1}{2}\right)$ e $\left(k-\frac{1}{2}\right)$ em contato com células E. Onde $n=\left(\frac{\sqrt{2}}{2}, 0,-\frac{\sqrt{2}}{2}\right), m 1=\left(\frac{\sqrt{2}}{2}, 0, \frac{\sqrt{2}}{2}\right)$ e $m 2=$ $(0,1,0)$.

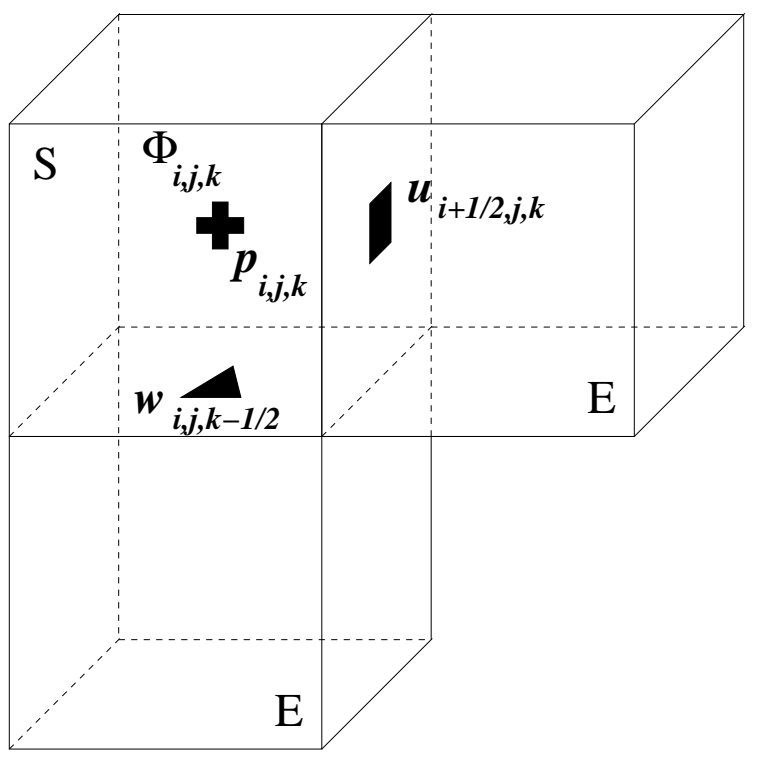

Figura B.13: Célula S com as faces $\left(i+\frac{1}{2}\right)$ e $\left(k-\frac{1}{2}\right)$ em contato com faces de células vazias.

$$
\begin{gathered}
\tilde{p}=\frac{1}{R e}\left[\frac{\partial u}{\partial x}+\frac{\partial w}{\partial z}-\left(\frac{\partial u}{\partial z}+\frac{\partial w}{\partial x}\right)+\frac{1}{2}\left(\Phi^{x x}+\Phi^{z z}\right)-\Phi^{x z}\right], \\
\frac{\partial u}{\partial x}-\frac{\partial w}{\partial z}=-\frac{1}{2}\left(\Phi^{x x}-\Phi^{z z}\right)
\end{gathered}
$$

Discretizando (B.89)

$$
\frac{u_{i+\frac{1}{2}, j, k}-u_{i-\frac{1}{2}, j, k}}{\delta x}-\frac{w_{i, j, k+\frac{1}{2}}-w_{i, j, k-\frac{1}{2}}}{\delta z}=-\frac{1}{2}\left(\Phi_{i, j, k}^{x x}-\Phi_{i, j, k}^{z z}\right)
$$

e pela equação da continuidade (1.75) temos

$$
\frac{u_{i+\frac{1}{2}, j, k}-u_{i-\frac{1}{2}, j, k}}{\delta x}+\frac{w_{i, j, k+\frac{1}{2}}-w_{i, j, k-\frac{1}{2}}}{\delta z}=-\left(\frac{v_{i, j+\frac{1}{2}, k}-v_{i, j-\frac{1}{2}, k}}{\delta y}\right) .
$$


Temos como solução

$$
u_{i+\frac{1}{2}, j, k}=u_{i-\frac{1}{2}, j, k}-\frac{1}{2} \frac{\delta x}{\delta y}\left(v_{i, j+\frac{1}{2}, k}-v_{i, j-\frac{1}{2}, k}\right)-\frac{\delta x}{4}\left(\Phi_{i, j, k}^{x x}-\Phi_{i, j, k}^{z z}\right)
$$

e

$$
w_{i, j, k-\frac{1}{2}}=w_{i, j, k+\frac{1}{2}}+\frac{1}{2} \frac{\delta z}{\delta y}\left(v_{i, j+\frac{1}{2}, k}-v_{i, j-\frac{1}{2}, k}\right)-\frac{\delta z}{4}\left(\Phi_{i, j, k}^{x x}-\Phi_{i, j, k}^{z z}\right)
$$

Depois de calcudados $u_{i+\frac{1}{2}, j, k}$ e $w_{i, j, k-\frac{1}{2}}$, a pressão no centro da célula de superfície é obtida por

$$
\begin{aligned}
\tilde{p}= & \frac{1}{R e}\left[\frac{u_{i+\frac{1}{2}, j, k}-u_{i-\frac{1}{2}, j, k}}{\delta x}+\frac{w_{i, j, k+\frac{1}{2}}-w_{i, j, k-\frac{1}{2}}}{\delta z}\right. \\
& -\frac{1}{2}\left(\frac{u_{i+\frac{1}{2}, j, k+1}+u_{i-\frac{1}{2}, j, k+1}-u_{i+\frac{1}{2}, j, k}-u_{i-\frac{1}{2}, j, k}}{\delta z}\right. \\
& \left.+\frac{w_{i, j, k+\frac{1}{2}}+w_{i, j, k-\frac{1}{2}}-w_{i-1, j, k+\frac{1}{2}}-w_{i-1, j, k-\frac{1}{2}}}{\delta x}\right) \\
& \left.+\frac{1}{2}\left(\Phi_{i, j, k}^{x x}+\Phi_{i, j, k}^{z z}\right)-\Phi_{i, j, k}^{x z}\right] .
\end{aligned}
$$

B.2.9 Células $S$ possuem apenas a face $\left(j+\frac{1}{2}\right)$ e $\left(k-\frac{1}{2}\right)$ em contato com células $\mathbf{E}$. Onde $n=\left(0, \frac{\sqrt{2}}{2},-\frac{\sqrt{2}}{2}\right), m 1=\left(0, \frac{\sqrt{2}}{2}, \frac{\sqrt{2}}{2}\right)$ e $m 2=$ $(1,0,0)$.

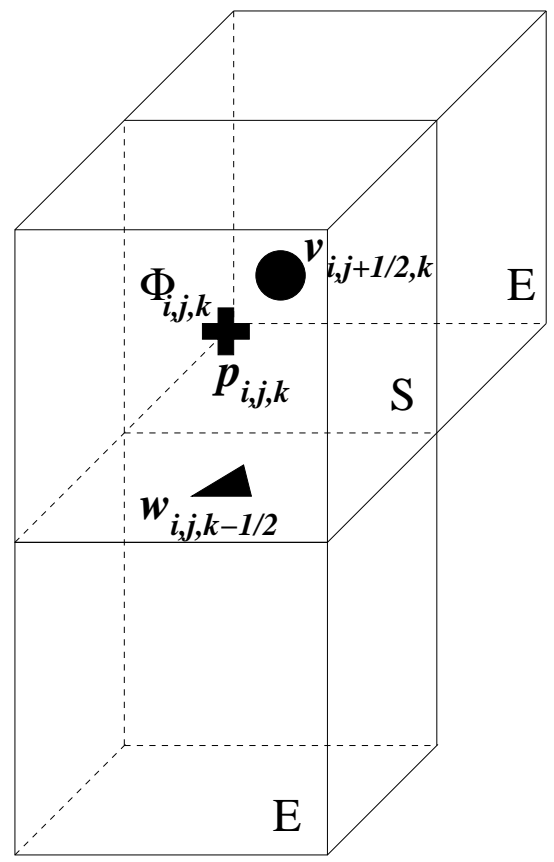

Figura B.14: Célula S com as faces $\left(j+\frac{1}{2}\right)$ e $\left(k-\frac{1}{2}\right)$ em contato com faces de células vazias.

$$
\tilde{p}=\frac{1}{R e}\left[\frac{\partial v}{\partial y}+\frac{\partial w}{\partial z}-\left(\frac{\partial v}{\partial z}+\frac{\partial w}{\partial y}\right)+\frac{1}{2}\left(\Phi^{y y}+\Phi^{z z}\right)-\Phi^{y z}\right]
$$




$$
\frac{\partial v}{\partial y}-\frac{\partial w}{\partial z}=-\frac{1}{2}\left(\Phi^{y y}-\Phi^{z z}\right)
$$

Discretizando (B.96)

$$
\frac{v_{i, j+\frac{1}{2}, k}-v_{i, j-\frac{1}{2}, k}}{\delta y}-\frac{w_{i, j, k+\frac{1}{2}}-w_{i, j, k-\frac{1}{2}}}{\delta z}=-\frac{1}{2}\left(\Phi_{i, j, k}^{y y}-\Phi_{i, j, k}^{z z}\right)
$$

e pela equação da continuidade (1.75) temos

$$
\frac{v_{i, j+\frac{1}{2}, k}-v_{i, j-\frac{1}{2}, k}}{\delta y}+\frac{w_{i, j, k+\frac{1}{2}}-w_{i, j, k-\frac{1}{2}}}{\delta z}=-\left(\frac{u_{i+\frac{1}{2}, j, k}-u_{i-\frac{1}{2}, j, k}}{\delta x}\right) .
$$

Temos como solução

$$
v_{i, j+\frac{1}{2}, k}=v_{i, j-\frac{1}{2}, k}-\frac{1}{2} \frac{\delta y}{\delta x}\left(u_{i+\frac{1}{2}, j, k}-u_{i-\frac{1}{2}, j, k}\right)-\frac{\delta y}{4}\left(\Phi_{i, j, k}^{y y}-\Phi_{i, j, k}^{z z}\right)
$$

e

$$
w_{i, j, k-\frac{1}{2}}=w_{i, j, k+\frac{1}{2}}+\frac{1}{2} \frac{\delta z}{\delta x}\left(u_{i+\frac{1}{2}, j, k}-u_{i-\frac{1}{2}, j, k}\right)-\frac{\delta z}{4}\left(\Phi_{i, j, k}^{y y}-\Phi_{i, j, k}^{z z}\right)
$$

Depois de calcudados $v_{i, j+\frac{1}{2}, k}$ e $w_{i, j, k-\frac{1}{2}}$, a pressão no centro da célula de superfície é obtida por

$$
\begin{aligned}
\tilde{p}= & \frac{1}{R e}\left[\frac{v_{i, j+\frac{1}{2}, k}-v_{i, j-\frac{1}{2}, k}}{\delta y}+\frac{w_{i, j, k+\frac{1}{2}}-w_{i, j, k-\frac{1}{2}}}{\delta z}\right. \\
& -\frac{1}{2}\left(\frac{v_{i, j+\frac{1}{2}, k+1}+v_{i, j-\frac{1}{2}, k+1}-v_{i, j+\frac{1}{2}, k}-v_{i, j-\frac{1}{2}, k}}{\delta z}\right. \\
& \left.+\frac{w_{i, j, k+\frac{1}{2}}+w_{i, j, k-\frac{1}{2}}-w_{i, j-1, k+\frac{1}{2}}-w_{i, j-1, k-\frac{1}{2}}}{\delta y}\right) \\
& \left.+\frac{1}{2}\left(\Phi_{i, j, k}^{y y}+\Phi_{i, j, k}^{z z}\right)-\Phi_{i, j, k}^{y z}\right] .
\end{aligned}
$$

B.2.10 Células $\mathrm{S}$ possuem apenas a face $\left(i-\frac{1}{2}\right)$ e $\left(k-\frac{1}{2}\right)$ em contato com células E. Onde $n=\left(-\frac{\sqrt{2}}{2}, 0,-\frac{\sqrt{2}}{2}\right), m 1=\left(\frac{\sqrt{2}}{2}, 0,-\frac{\sqrt{2}}{2}\right)$ e $m 2=(0,1,0)$.

$$
\begin{gathered}
\tilde{p}=\frac{1}{R e}\left[\frac{\partial u}{\partial x}+\frac{\partial w}{\partial z}+\left(\frac{\partial u}{\partial z}+\frac{\partial w}{\partial x}\right)+\frac{1}{2}\left(\Phi^{x x}+\Phi^{z z}\right)+\Phi^{x z}\right] \\
-\frac{\partial u}{\partial x}+\frac{\partial w}{\partial z}=-\frac{1}{2}\left(-\Phi^{x x}+\Phi^{z z}\right)
\end{gathered}
$$

Discretizando (B.103)

$$
-\frac{u_{i+\frac{1}{2}, j, k}-u_{i-\frac{1}{2}, j, k}}{\delta x}+\frac{w_{i, j, k+\frac{1}{2}}-w_{i, j, k-\frac{1}{2}}}{\delta z}=-\frac{1}{2}\left(-\Phi_{i, j, k}^{x x}+\Phi_{i, j, k}^{z z}\right)
$$

e pela equação da continuidade (1.75) temos

$$
\frac{u_{i+\frac{1}{2}, j, k}-u_{i-\frac{1}{2}, j, k}}{\delta x}+\frac{w_{i, j, k+\frac{1}{2}}-w_{i, j, k-\frac{1}{2}}}{\delta z}=-\left(\frac{v_{i, j+\frac{1}{2}, k}-v_{i, j-\frac{1}{2}, k}}{\delta y}\right) .
$$




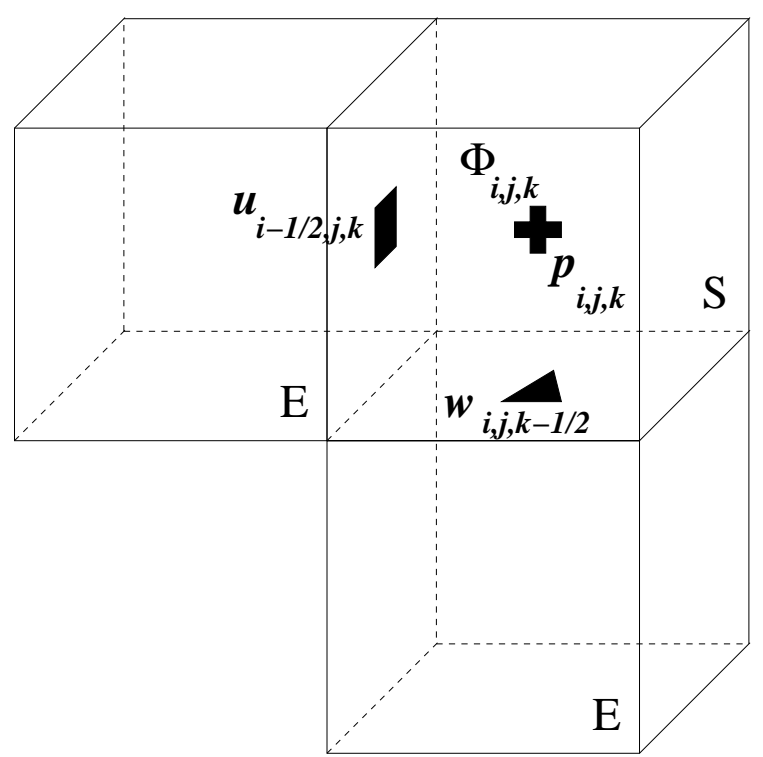

Figura B.15: Célula S com as faces $\left(i-\frac{1}{2}\right)$ e $\left(k-\frac{1}{2}\right)$ em contato com faces de células vazias.

Temos como solução

$$
u_{i-\frac{1}{2}, j, k}=u_{i+\frac{1}{2}, j, k}+\frac{1}{2} \frac{\delta x}{\delta y}\left(v_{i, j+\frac{1}{2}, k}-v_{i, j-\frac{1}{2}, k}\right)-\frac{\delta x}{4}\left(-\Phi_{i, j, k}^{x x}+\Phi_{i, j, k}^{z z}\right)
$$

e

$$
w_{i, j, k-\frac{1}{2}}=w_{i, j, k+\frac{1}{2}}+\frac{1}{2} \frac{\delta z}{\delta y}\left(v_{i, j+\frac{1}{2}, k}-v_{i, j-\frac{1}{2}, k}\right)+\frac{\delta z}{4}\left(-\Phi_{i, j, k}^{x x}+\Phi_{i, j, k}^{z z}\right)
$$

Depois de calcudados $u_{i-\frac{1}{2}, j, k}$ e $w_{i, j, k-\frac{1}{2}}$, a pressão no centro da célula de superfície é obtida por

$$
\begin{aligned}
\tilde{p}= & \frac{1}{R e}\left[\frac{u_{i+\frac{1}{2}, j, k}-u_{i-\frac{1}{2}, j, k}}{\delta x}+\frac{w_{i, j, k+\frac{1}{2}}-w_{i, j, k-\frac{1}{2}}}{\delta z}\right. \\
& +\frac{1}{2}\left(\frac{u_{i+\frac{1}{2}, j, k+1}+u_{i-\frac{1}{2}, j, k+1}-u_{i+\frac{1}{2}, j, k}-u_{i-\frac{1}{2}, j, k}}{\delta z}\right. \\
& \left.+\frac{w_{i+1, j, k+\frac{1}{2}}+w_{i+1, j, k-\frac{1}{2}}-w_{i, j, k+\frac{1}{2}}-w_{i, j, k-\frac{1}{2}}}{\delta x}\right) \\
& \left.+\frac{1}{2}\left(\Phi_{i, j, k}^{x x}+\Phi_{i, j, k}^{z z}\right)+\Phi_{i, j, k}^{x z}\right] .
\end{aligned}
$$

B.2.11 Células S possuem apenas a face $\left(j-\frac{1}{2}\right)$ e $\left(k-\frac{1}{2}\right)$ em contato com células $\mathbf{E}$. Onde $n=\left(0,-\frac{\sqrt{2}}{2},-\frac{\sqrt{2}}{2}\right), m 1=\left(0, \frac{\sqrt{2}}{2},-\frac{\sqrt{2}}{2}\right)$ e $m 2=(1,0,0)$.

$$
\begin{gathered}
\tilde{p}=\frac{1}{R e}\left[\frac{\partial v}{\partial y}+\frac{\partial w}{\partial z}+\left(\frac{\partial v}{\partial z}+\frac{\partial w}{\partial y}\right)+\frac{1}{2}\left(\Phi^{y y}+\Phi^{z z}\right)+\Phi^{y z}\right] \\
-\frac{\partial v}{\partial y}+\frac{\partial w}{\partial z}=-\frac{1}{2}\left(-\Phi^{y y}+\Phi^{z z}\right)
\end{gathered}
$$




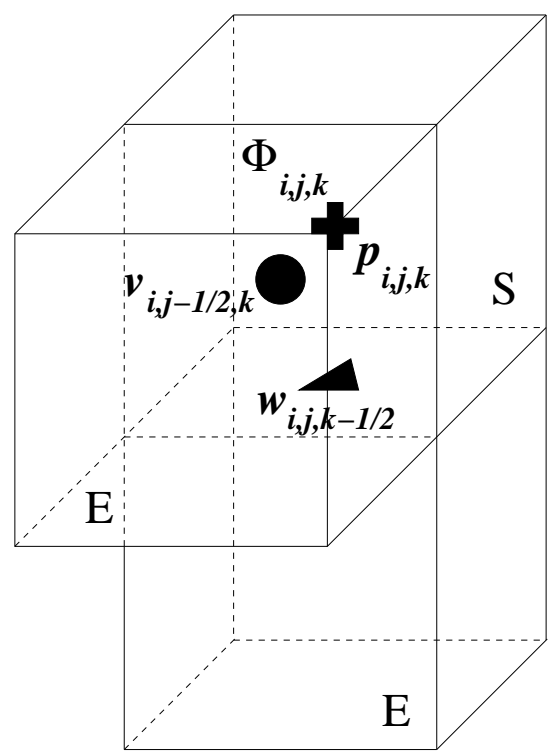

Figura B.16: Célula S com as faces $\left(j-\frac{1}{2}\right)$ e $\left(k-\frac{1}{2}\right)$ em contato com faces de células vazias.

Discretizando (B.110)

$$
-\frac{v_{i, j+\frac{1}{2}, k}-v_{i, j-\frac{1}{2}, k}}{\delta y}+\frac{w_{i, j, k+\frac{1}{2}}-w_{i, j, k-\frac{1}{2}}}{\delta z}=-\frac{1}{2}\left(-\Phi_{i, j, k}^{y y}+\Phi_{i, j, k}^{z z}\right)
$$

e pela equação da continuidade (1.75) temos

$$
\frac{v_{i, j+\frac{1}{2}, k}-v_{i, j-\frac{1}{2}, k}}{\delta y}+\frac{w_{i, j, k+\frac{1}{2}}-w_{i, j, k-\frac{1}{2}}}{\delta z}=-\left(\frac{u_{i+\frac{1}{2}, j, k}-u_{i-\frac{1}{2}, j, k}}{\delta x}\right) .
$$

Temos como solução

$$
v_{i, j-\frac{1}{2}, k}=v_{i, j+\frac{1}{2}, k}+\frac{1}{2} \frac{\delta y}{\delta x}\left(u_{i+\frac{1}{2}, j, k}-u_{i-\frac{1}{2}, j, k}\right)-\frac{\delta y}{4}\left(-\Phi_{i, j, k}^{y y}+\Phi_{i, j, k}^{z z}\right)
$$

e

$$
w_{i, j, k-\frac{1}{2}}=w_{i, j, k+\frac{1}{2}}+\frac{1}{2} \frac{\delta z}{\delta x}\left(u_{i+\frac{1}{2}, j, k}-u_{i-\frac{1}{2}, j, k}\right)+\frac{\delta z}{4}\left(-\Phi_{i, j, k}^{y y}+\Phi_{i, j, k}^{z z}\right)
$$

Depois de calcudados $v_{i, j+\frac{1}{2}, k}$ e $w_{i, j, k-\frac{1}{2}}$, a pressão no centro da célula de superfície é obtida por

$$
\begin{aligned}
\tilde{p}= & \frac{1}{R e}\left[\frac{v_{i, j+\frac{1}{2}, k}-v_{i, j-\frac{1}{2}, k}}{\delta y}+\frac{w_{i, j, k+\frac{1}{2}}-w_{i, j, k-\frac{1}{2}}}{\delta z}\right. \\
& +\frac{1}{2}\left(\frac{v_{i, j+\frac{1}{2}, k+1}+v_{i, j-\frac{1}{2}, k+1}-v_{i, j+\frac{1}{2}, k}-v_{i, j-\frac{1}{2}, k}}{\delta z}\right. \\
& \left.+\frac{w_{i, j+1, k+\frac{1}{2}}+w_{i, j+1, k-\frac{1}{2}}-w_{i, j, k+\frac{1}{2}}-w_{i, j, k-\frac{1}{2}}}{\delta y}\right) \\
& \left.+\frac{1}{2}\left(\Phi_{i, j, k}^{y y}+\Phi_{i, j, k}^{z z}\right)+\Phi_{i, j, k}^{y z}\right] .
\end{aligned}
$$




\section{B.3 Superfície plana com inclinação de $60^{\circ}$}

B.3.1 Células $\mathbf{S}$ possuem as faces $\left(i-\frac{1}{2}\right),\left(j+\frac{1}{2}\right)$ e $\left(k+\frac{1}{2}\right)$ em contato com células $E$.

$$
\mathbf{n}=\left(-\frac{\sqrt{3}}{3}, \frac{\sqrt{3}}{3}, \frac{\sqrt{3}}{3}\right), \mathbf{m} \mathbf{1}=\left(0, \frac{\sqrt{2}}{2},-\frac{\sqrt{2}}{2}\right), \mathbf{m} \mathbf{2}=\left(\frac{\sqrt{6}}{3}, \frac{\sqrt{6}}{6}, \frac{\sqrt{6}}{6}\right)
$$

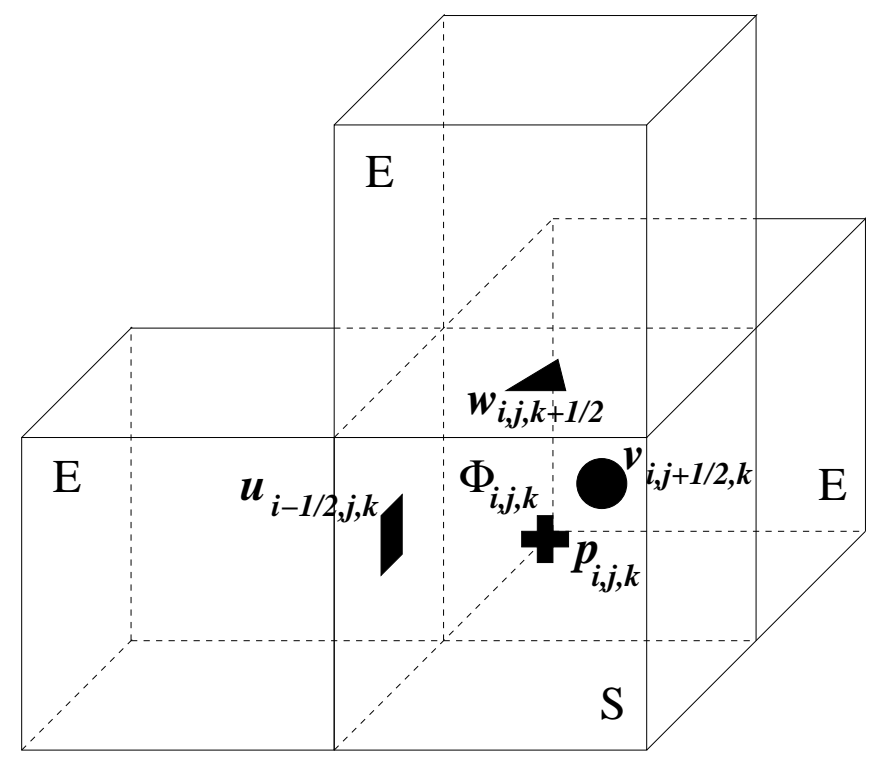

Figura B.17: Célula S com as faces $\left(i-\frac{1}{2}\right),\left(j+\frac{1}{2}\right)$ e $\left(k+\frac{1}{2}\right)$ em contato com faces de células vazias.

$$
\begin{gathered}
\tilde{p}=\frac{1}{3 R e}\left[-2\left(\frac{\partial u}{\partial y}+\frac{\partial v}{\partial x}\right)-2\left(\frac{\partial u}{\partial z}+\frac{\partial w}{\partial x}\right)+2\left(\frac{\partial v}{\partial z}+\frac{\partial w}{\partial y}\right)\right. \\
\left.+\Phi^{x x}+\Phi^{y y}+\Phi^{z z}+2\left(-\Phi^{x y}-\Phi^{x z}+\Phi^{y z}\right)\right] \\
2 \frac{\partial v}{\partial y}-2 \frac{\partial w}{\partial z}-\left(\frac{\partial u}{\partial y}+\frac{\partial v}{\partial x}\right)+\left(\frac{\partial u}{\partial z}+\frac{\partial w}{\partial x}\right)=-\left[\Phi^{y y}-\Phi^{z z}-\Phi^{x y}+\Phi^{x z}\right], \\
-4 \frac{\partial u}{\partial x}+2 \frac{\partial v}{\partial y}+2 \frac{\partial w}{\partial z}+\left(\frac{\partial u}{\partial y}+\frac{\partial v}{\partial x}\right)+\left(\frac{\partial u}{\partial z}+\frac{\partial w}{\partial x}\right)+2\left(\frac{\partial v}{\partial z}+\frac{\partial w}{\partial y}\right) \\
=-\left[-2 \Phi^{x x}+\Phi^{y y}+\Phi^{z z}+\Phi^{x y}+\Phi^{x z}+2 \Phi^{y z}\right]
\end{gathered}
$$

Pela equação da continuidade

$$
\frac{\partial u}{\partial x}+\frac{\partial v}{\partial y}+\frac{\partial w}{\partial z}=0
$$

Agora, adicionando as equações (B.117) e (B.118) obtém-se

$$
-2 \frac{\partial u}{\partial x}+2 \frac{\partial v}{\partial y}+\left(\frac{\partial u}{\partial z}+\frac{\partial w}{\partial x}\right)+\left(\frac{\partial v}{\partial z}+\frac{\partial w}{\partial y}\right)=\left[\Phi^{x x}-\Phi^{y y}-\Phi^{x z}-\Phi^{y z}\right] .
$$


Com relação a figura 3.9 , os valores de $u_{i-\frac{1}{2}, j, k}, v_{i, j+\frac{1}{2}, k}, w_{i, j, k+\frac{1}{2}}$ e $\tilde{p}_{i, j, k}$ são requeridos e podem ser calculados pelas equações (B.117),(B.119), (B.120) e (B.116), como segue.

Aplicando a equação de conservação de massa no centro da célula, obtemos

$$
\frac{u_{i+\frac{1}{2}, j, k}-u_{i-\frac{1}{2}, j, k}}{\delta x}+\frac{v_{i, j+\frac{1}{2}, k}-v_{i, j-\frac{1}{2}, k}}{\delta y}+\frac{w_{i, j, k+\frac{1}{2}}-w_{i, j, k-\frac{1}{2}}}{\delta z}=0
$$

que pode ser escrita como

$$
-u_{i-\frac{1}{2}, j, k}+\frac{\delta x}{\delta y} v_{i, j+\frac{1}{2}, k}+\frac{\delta x}{\delta z} w_{i, j, k+\frac{1}{2}}=b_{3}
$$

onde

$$
b_{3}=-u_{i+\frac{1}{2}, j, k}+\frac{\delta x}{\delta y} v_{i, j-\frac{1}{2}, k}+\frac{\delta x}{\delta z} w_{i, j, k-\frac{1}{2}}
$$

Agora, equação (B.117) aplicada no centro da célula fornece

$$
\begin{aligned}
& 2\left(\frac{v_{i, j+\frac{1}{2}, k}-v_{i, j-\frac{1}{2}, k}}{\delta y}\right)-2\left(\frac{w_{i, j, k+\frac{1}{2}}-w_{i, j, k-\frac{1}{2}}}{\delta z}\right) \\
& -\frac{1}{2}\left(\frac{u_{i+\frac{1}{2}, j, k}+u_{i-\frac{1}{2}, j, k}-u_{i+\frac{1}{2}, j-1, k}-u_{i-\frac{1}{2}, j-1, k}}{\delta y}\right. \\
& \left.+\frac{v_{i+1, j+\frac{1}{2}, k}+v_{i+1, j-\frac{1}{2}, k}-v_{i, j+\frac{1}{2}, k}-v_{i, j-\frac{1}{2}, k}}{\delta x}\right) \\
& +\frac{1}{2}\left(\frac{u_{i+\frac{1}{2}, j, k}+u_{i-\frac{1}{2}, j, k}-u_{i+\frac{1}{2}, j, k-1}-u_{i-\frac{1}{2}, j, k-1}}{\delta z}\right) \\
& \left.+\frac{w_{i+1, j, k+\frac{1}{2}}+w_{i+1, j, k-\frac{1}{2}}-w_{i, j, k+\frac{1}{2}}-w_{i, j, k-\frac{1}{2}}}{\delta x}\right) \\
& =-\left[\Phi_{i, j, k}^{y y}-\Phi_{i, j, k}^{z z}-\Phi_{i, j, k}^{x y}+\Phi_{i, j, k}^{x z}\right]
\end{aligned}
$$

que pode ser reescrita como (multiplicando por 2 e por $\delta x$ )

$$
\left(\frac{\delta x}{\delta z}-\frac{\delta x}{\delta y}\right) u_{i-\frac{1}{2}, j, k}+\left(4 \frac{\delta x}{\delta y}+1\right) v_{i, j+\frac{1}{2}, k}-\left(4 \frac{\delta x}{\delta z}+1\right) w_{i, j, k+\frac{1}{2}}=b_{2}
$$

onde

$$
\begin{aligned}
b_{2}= & 4 \frac{\delta x}{\delta y} v_{i, j-\frac{1}{2}, k}-4 \frac{\delta x}{\delta z} w_{i, j, k-\frac{1}{2}}+\left(\frac{\delta x}{\delta y}\right)\left(u_{i+\frac{1}{2}, j, k}-u_{i+\frac{1}{2}, j-1, k}-u_{i-\frac{1}{2}, j-1, k}\right) \\
& +\left(v_{i+1, j+\frac{1}{2}, k}+v_{i+1, j-\frac{1}{2}, k}-v_{i, j-\frac{1}{2}, k}\right) \\
& -\left(\frac{\delta x}{\delta z}\right)\left(u_{i+\frac{1}{2}, j, k}-u_{i+\frac{1}{2}, j, k-1}-u_{i-\frac{1}{2}, j, k-1}\right) \\
& -\left(w_{i+1, j, k+\frac{1}{2}}+w_{i+1, j, k-\frac{1}{2}}-w_{i, j, k-\frac{1}{2}}\right) \\
& -2 \delta x\left[\Phi_{i, j, k}^{y y}-\Phi_{i, j, k}^{z z}-\Phi_{i, j, k}^{x y}+\Phi_{i, j, k}^{x z}\right] .
\end{aligned}
$$


Analogamente, discretizando (B.120) da célula de superfície na posição $(i, j, k)$, obtemos

$$
\begin{aligned}
& -2\left(\frac{u_{i+\frac{1}{2}, j, k}-u_{i-\frac{1}{2}, j, k}}{\delta x}\right)+2\left(\frac{v_{i, j+\frac{1}{2}, k}-v_{i, j-\frac{1}{2}, k}}{\delta y}\right) \\
& +\frac{1}{2}\left(\frac{u_{i+\frac{1}{2}, j, k}+u_{i-\frac{1}{2}, j, k}-u_{i+\frac{1}{2}, j, k-1}-u_{i-\frac{1}{2}, j, k-1}}{\delta z}\right. \\
& \left.+\frac{w_{i+1, j, k+\frac{1}{2}}+w_{i+1, j, k-\frac{1}{2}}-w_{i, j, k+\frac{1}{2}}-w_{i, j, k-\frac{1}{2}}}{\delta x}\right) \\
& +\frac{1}{2}\left(\frac{v_{i, j+\frac{1}{2}, k}+v_{i, j-\frac{1}{2}, k}-v_{i, j+\frac{1}{2}, k-1}-v_{i, j-\frac{1}{2}, k-1}}{\delta z}\right) \\
& \left.+\frac{w_{i, j, k+\frac{1}{2}}+w_{i, j, k-\frac{1}{2}}-w_{i, j-1, k+\frac{1}{2}}-w_{i, j-1, k-\frac{1}{2}}}{\delta y}\right) \\
& =\left[\Phi_{i, j, k}^{x x}-\Phi_{i, j, k}^{y y}-\Phi_{i, j, k}^{x z}-\Phi_{i, j, k}^{y z}\right]
\end{aligned}
$$

a qual reescrevemos como

$$
\left(4+\frac{\delta x}{\delta z}\right) u_{i-\frac{1}{2}, j, k}+\left(4 \frac{\delta x}{\delta y}+\frac{\delta x}{\delta z}\right) v_{i, j+\frac{1}{2}, k}+\left(\frac{\delta x}{\delta y}-1\right) w_{i, j, k+\frac{1}{2}}=b_{1}
$$

onde

$$
\begin{aligned}
b_{1}= & 4 u_{i+\frac{1}{2}, j, k}+4 \frac{\delta x}{\delta y} v_{i, j-\frac{1}{2}, k}-\left(\frac{\delta x}{\delta z}\right)\left(u_{i+\frac{1}{2}, j, k}-u_{i+\frac{1}{2}, j, k-1}-u_{i-\frac{1}{2}, j, k-1}\right) \\
& -\left(w_{i+1, j, k+\frac{1}{2}}+w_{i+1, j, k-\frac{1}{2}}-w_{i, j, k-\frac{1}{2}}\right) \\
& -\left(\frac{\delta x}{\delta z}\right)\left(v_{i, j-\frac{1}{2}, k}-v_{i, j+\frac{1}{2}, k-1}-v_{i, j-\frac{1}{2}, k-1}\right) \\
& -\left(\frac{\delta x}{\delta y}\right)\left(w_{i, j, k-\frac{1}{2}}-w_{i, j-1, k+\frac{1}{2}}-w_{i, j-1, k-\frac{1}{2}}\right) \\
& +2 \delta x\left[\Phi_{i, j, k}^{x x}-\Phi_{i, j, k}^{y y}-\Phi_{i, j, k}^{x z}-\Phi_{i, j, k}^{y z}\right] .
\end{aligned}
$$

As equações (B.124), (B.123) e (B.122) fornecem um sistema linear $(3 \times 3)$ para as velocidades $u_{i-\frac{1}{2}, j, k}, v_{i, j+\frac{1}{2}, k}, w_{i, j, k+\frac{1}{2}}$ que pode ser escrito na forma matricial como:

$$
\left[\begin{array}{ccc}
\left(4+\frac{\delta x}{\delta z}\right) & \left(4 \frac{\delta x}{\delta y}+\frac{\delta x}{\delta z}\right) & \left(\frac{\delta x}{\delta y}-1\right) \\
\left(\frac{\delta x}{\delta z}-\frac{\delta x}{\delta y}\right) & \left(4 \frac{\delta x}{\delta y}+1\right) & -\left(4 \frac{\delta x}{\delta z}+1\right) \\
-1 & \frac{\delta x}{\delta y} & \frac{\delta x}{\delta z}
\end{array}\right]\left[\begin{array}{c}
u_{i-\frac{1}{2}, j, k} \\
v_{i, j+\frac{1}{2}, k} \\
w_{i, j, k+\frac{1}{2}}
\end{array}\right]=\left[\begin{array}{l}
b_{1} \\
b_{2} \\
b_{3}
\end{array}\right]
$$

Esse sistema pode ser resolvido facilmente pelo método de eliminação de Gauss. Com os valores de $u_{i-\frac{1}{2}, j, k}, v_{i, j+\frac{1}{2}, k}$ e $w_{i, j, k+\frac{1}{2}}$ calculados a pressão é obtida pela discretização de 
(B.116), no centro da célula de superfície, obtendo,

$$
\begin{aligned}
\tilde{p}_{i, j, k}= & \frac{1}{3 R e}\left[-\left(\frac{u_{i+\frac{1}{2}, j, k}+u_{i-\frac{1}{2}, j, k}-u_{i+\frac{1}{2}, j-1, k}-u_{i-\frac{1}{2}, j-1, k}}{\delta y}\right.\right. \\
& \left.+\frac{v_{i+1, j+\frac{1}{2}, k}+v_{i+1, j-\frac{1}{2}, k}-v_{i, j+\frac{1}{2}, k}-v_{i, j-\frac{1}{2}, k}}{\delta x}\right) \\
& -\left(\frac{u_{i+\frac{1}{2}, j, k}+u_{i-\frac{1}{2}, j, k}-u_{i+\frac{1}{2}, j, k-1}-u_{i-\frac{1}{2}, j, k-1}}{\delta z}\right. \\
& \left.+\frac{w_{i+1, j, k+\frac{1}{2}}+w_{i+1, j, k-\frac{1}{2}}-w_{i, j, k+\frac{1}{2}}-w_{i, j, k-\frac{1}{2}}}{\delta x}\right) \\
& +\left(\frac{v_{i, j+\frac{1}{2}, k}+v_{i, j-\frac{1}{2}, k}-v_{i, j+\frac{1}{2}, k-1}-v_{i, j-\frac{1}{2}, k-1}}{\delta z}\right. \\
& \left.\left.+\frac{w_{i, j, k+\frac{1}{2}}+w_{i, j, k-\frac{1}{2}}-w_{i, j-1, k+\frac{1}{2}}-w_{i, j-1, k-\frac{1}{2}}}{\delta y}\right)\right] \\
& +\frac{1}{3 R e}\left[\Phi_{i, j, k}^{x x}+\Phi_{i, j, k}^{y y}+\Phi_{i, j, k}^{z z}+2\left(-\Phi_{i, j, k}^{x y}-\Phi_{i, j, k}^{x z}+\Phi_{i, j, k}^{y z}\right)\right] .
\end{aligned}
$$

B.3.2 Células S possuem as faces $\left(i+\frac{1}{2}\right),\left(j-\frac{1}{2}\right)$ e $\left(k+\frac{1}{2}\right)$ em contato com células $\mathrm{E}$.

$$
\mathbf{n}=\left(\frac{\sqrt{3}}{3},-\frac{\sqrt{3}}{3}, \frac{\sqrt{3}}{3}\right), \mathbf{m} \mathbf{1}=\left(\frac{\sqrt{2}}{2}, 0,-\frac{\sqrt{2}}{2}\right), \mathbf{m} \mathbf{2}=\left(\frac{\sqrt{6}}{6}, \frac{\sqrt{6}}{3}, \frac{\sqrt{6}}{6}\right)
$$

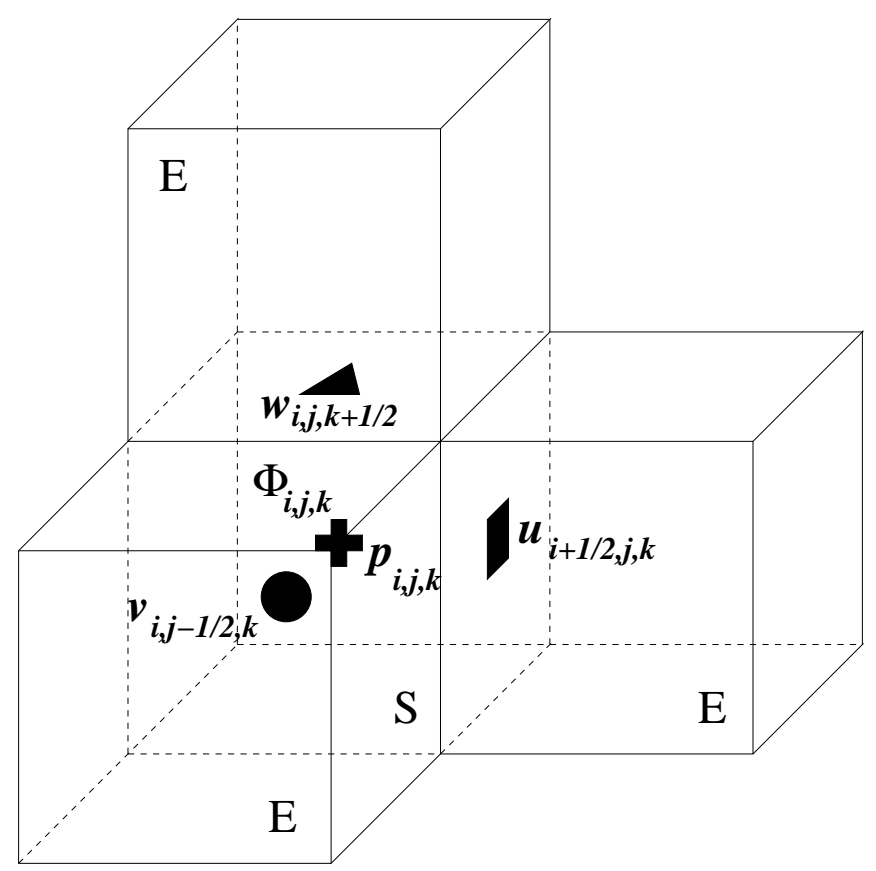

Figura B.18: Célula S com as faces $\left(i+\frac{1}{2}\right),\left(j-\frac{1}{2}\right)$ e $\left(k+\frac{1}{2}\right)$ em contato com faces de células vazias. 


$$
\begin{gathered}
\tilde{p}=\frac{1}{3 R e}\left[-2\left(\frac{\partial u}{\partial y}+\frac{\partial v}{\partial x}\right)+2\left(\frac{\partial u}{\partial z}+\frac{\partial w}{\partial x}\right)-2\left(\frac{\partial v}{\partial z}+\frac{\partial w}{\partial y}\right)\right. \\
\left.+\Phi^{x x}+\Phi^{y y}+\Phi^{z z}+2\left(-\Phi^{x y}+\Phi^{x z}-\Phi^{y z}\right)\right] \\
2 \frac{\partial u}{\partial x}-2 \frac{\partial w}{\partial z}-\left(\frac{\partial u}{\partial y}+\frac{\partial v}{\partial x}\right)+\left(\frac{\partial v}{\partial z}+\frac{\partial w}{\partial y}\right)=-\left[\Phi^{x x}-\Phi^{z z}-\Phi^{x y}+\Phi^{y z}\right] \\
2 \frac{\partial u}{\partial x}-4 \frac{\partial v}{\partial y}+2 \frac{\partial w}{\partial z}+\left(\frac{\partial u}{\partial y}+\frac{\partial v}{\partial x}\right)+2\left(\frac{\partial u}{\partial z}+\frac{\partial w}{\partial x}\right)+\left(\frac{\partial v}{\partial z}+\frac{\partial w}{\partial y}\right) \\
=-\left[\Phi^{x x}-2 \Phi^{y y}+\Phi^{z z}+\Phi^{x y}+2 \Phi^{x z}+\Phi^{y z}\right]
\end{gathered}
$$

Agora, adicionando as equações (B.128) e (B.129) obtém-se

$$
2 \frac{\partial u}{\partial x}-2 \frac{\partial v}{\partial y}+\left(\frac{\partial u}{\partial z}+\frac{\partial w}{\partial x}\right)+\left(\frac{\partial v}{\partial z}+\frac{\partial w}{\partial y}\right)=-\left[\Phi^{x x}-\Phi^{y y}+\Phi^{x z}+\Phi^{y z}\right]
$$

Pela discretização da equação da continuidade obtemos

$$
u_{i+\frac{1}{2}, j, k}-\frac{\delta x}{\delta y} v_{i, j-\frac{1}{2}, k}+\frac{\delta x}{\delta z} w_{i, j, k+\frac{1}{2}}=b_{3}
$$

onde

$$
b_{3}=u_{i-\frac{1}{2}, j, k}-\frac{\delta x}{\delta y} v_{i, j+\frac{1}{2}, k}+\frac{\delta x}{\delta z} w_{i, j, k-\frac{1}{2}} .
$$

Agora, equação (B.128) aplicada no centro da célula fornece

$$
\begin{aligned}
& 2\left(\frac{u_{i+\frac{1}{2}, j, k}-u_{i-\frac{1}{2}, j, k}}{\delta x}\right)-2\left(\frac{w_{i, j, k+\frac{1}{2}}-w_{i, j, k-\frac{1}{2}}}{\delta z}\right) \\
& -\frac{1}{2}\left(\frac{u_{i+\frac{1}{2}, j+1, k}+u_{i-\frac{1}{2}, j+1, k}-u_{i+\frac{1}{2}, j, k}-u_{i-\frac{1}{2}, j, k}}{\delta y}\right. \\
& \left.+\frac{v_{i, j+\frac{1}{2}, k}+v_{i, j-\frac{1}{2}, k}-v_{i-1, j+\frac{1}{2}, k}-v_{i-1, j-\frac{1}{2}, k}}{\delta x}\right) \\
& +\frac{1}{2}\left(\frac{v_{i, j+\frac{1}{2}, k}+v_{i, j-\frac{1}{2}, k}-v_{i, j+\frac{1}{2}, k-1}-v_{i, j-\frac{1}{2}, k-1}}{\delta z}\right) \\
& \left.+\frac{w_{i, j+1, k+\frac{1}{2}}+w_{i, j+1, k-\frac{1}{2}}-w_{i, j, k+\frac{1}{2}}-w_{i, j, k-\frac{1}{2}}}{\delta y}\right) \\
& =-\left[\Phi_{i, j, k}^{x x}-\Phi_{i, j, k}^{z z}-\Phi_{i, j, k}^{x y}+\Phi_{i, j, k}^{y z}\right]
\end{aligned}
$$

que pode ser reescrita como (multiplicando por 2 e por $\delta x$ )

$$
\left(4+\frac{\delta x}{\delta y}\right) u_{i+\frac{1}{2}, j, k}+\left(\frac{\delta x}{\delta z}-1\right) v_{i, j-\frac{1}{2}, k}-\left(4 \frac{\delta x}{\delta z}+\frac{\delta x}{\delta y}\right) w_{i, j, k+\frac{1}{2}}=b_{2}
$$


onde

$$
\begin{aligned}
b_{2}= & 4 u_{i-\frac{1}{2}, j, k}-4 \frac{\delta x}{\delta z} w_{i, j, k-\frac{1}{2}}+\left(\frac{\delta x}{\delta y}\right)\left(u_{i+\frac{1}{2}, j+1, k}+u_{i-\frac{1}{2}, j+1, k}-u_{i-\frac{1}{2}, j, k}\right) \\
& +\left(v_{i, j+\frac{1}{2}, k}-v_{i-1, j+\frac{1}{2}, k}-v_{i-1, j-\frac{1}{2}, k}\right) \\
& -\left(\frac{\delta x}{\delta z}\right)\left(v_{i, j-\frac{1}{2}, k}-v_{i, j+\frac{1}{2}, k-1}-v_{i, j-\frac{1}{2}, k-1}\right) \\
& -\left(\frac{\delta x}{\delta y}\right)\left(w_{i, j+1, k+\frac{1}{2}}+w_{i, j+1, k-\frac{1}{2}}-w_{i, j, k-\frac{1}{2}}\right) \\
& -2 \delta x\left[\Phi_{i, j, k}^{x x}-\Phi_{i, j, k}^{z z}-\Phi_{i, j, k}^{x y}+\Phi_{i, j, k}^{y z}\right] .
\end{aligned}
$$

Analogamente, discretizando (B.130) da célula de superfície na posição $(i, j, k)$, obtemos

$$
\begin{aligned}
& 2\left(\frac{u_{i+\frac{1}{2}, j, k}-u_{i-\frac{1}{2}, j, k}}{\delta x}\right)-2\left(\frac{v_{i, j+\frac{1}{2}, k}-v_{i, j-\frac{1}{2}, k}}{\delta y}\right) \\
& +\frac{1}{2}\left(\frac{u_{i+\frac{1}{2}, j, k}+u_{i-\frac{1}{2}, j, k}-u_{i+\frac{1}{2}, j, k-1}-u_{i-\frac{1}{2}, j, k-1}}{\delta z}\right. \\
& \left.+\frac{w_{i, j, k+\frac{1}{2}}+w_{i, j, k-\frac{1}{2}}-w_{i-1, j, k+\frac{1}{2}}-w_{i-1, j, k-\frac{1}{2}}}{\delta x}\right) \\
& +\frac{1}{2}\left(\frac{v_{i, j+\frac{1}{2}, k}+v_{i, j-\frac{1}{2}, k}-v_{i, j+\frac{1}{2}, k-1}-v_{i, j-\frac{1}{2}, k-1}}{\delta z}\right) \\
& \left.+\frac{w_{i, j+1, k+\frac{1}{2}}+w_{i, j+1, k-\frac{1}{2}}-w_{i, j, k+\frac{1}{2}}-w_{i, j, k-\frac{1}{2}}}{\delta y}\right) \\
& =-\left[\Phi_{i, j, k}^{x x}-\Phi_{i, j, k}^{y y}+\Phi_{i, j, k}^{x z}+\Phi_{i, j, k}^{y z}\right]
\end{aligned}
$$

a qual reescrevemos como

$$
\left(4+\frac{\delta x}{\delta z}\right) u_{i+\frac{1}{2}, j, k}+\left(4 \frac{\delta x}{\delta y}+\frac{\delta x}{\delta z}\right) v_{i, j-\frac{1}{2}, k}+\left(1-\frac{\delta x}{\delta y}\right) w_{i, j, k+\frac{1}{2}}=b_{1}
$$

onde

$$
\begin{aligned}
b_{1}= & 4 u_{i-\frac{1}{2}, j, k}+4 \frac{\delta x}{\delta y} v_{i, j+\frac{1}{2}, k}-\left(\frac{\delta x}{\delta z}\right)\left(u_{i-\frac{1}{2}, j, k}-u_{i+\frac{1}{2}, j, k-1}-u_{i-\frac{1}{2}, j, k-1}\right) \\
& -\left(w_{i, j, k-\frac{1}{2}}-w_{i-1, j, k+\frac{1}{2}}-w_{i-1, j, k-\frac{1}{2}}\right) \\
& -\left(\frac{\delta x}{\delta z}\right)\left(v_{i, j+\frac{1}{2}, k}-v_{i, j+\frac{1}{2}, k-1}-v_{i, j-\frac{1}{2}, k-1}\right) \\
& -\left(\frac{\delta x}{\delta y}\right)\left(w_{i, j+1, k+\frac{1}{2}}+w_{i, j+1, k-\frac{1}{2}}-w_{i, j, k-\frac{1}{2}}\right) \\
& -2 \delta x\left[\Phi_{i, j, k}^{x x}-\Phi_{i, j, k}^{y y}+\Phi_{i, j, k}^{x z}+\Phi_{i, j, k}^{y z}\right] .
\end{aligned}
$$

As equações (B.133), (B.132) e (B.131) fornecem um sistema linear $(3 \times 3)$ para as velocidades $u_{i+\frac{1}{2}, j, k}, v_{i, j-\frac{1}{2}, k}, w_{i, j, k+\frac{1}{2}}$ que pode ser escrito na forma matricial como:

$$
\left[\begin{array}{ccc}
\left(4+\frac{\delta x}{\delta z}\right) & \left(4 \frac{\delta x}{\delta y}+\frac{\delta x}{\delta z}\right) & \left(1-\frac{\delta x}{\delta y}\right) \\
\left(4+\frac{\delta x}{\delta y}\right) & \left(\frac{\delta x}{\delta z}-1\right) & -\left(4 \frac{\delta x}{\delta z}+\frac{\delta x}{\delta y}\right) \\
1 & -\frac{\delta x}{\delta y} & \frac{\delta x}{\delta z}
\end{array}\right]\left[\begin{array}{c}
u_{i+\frac{1}{2}, j, k} \\
v_{i, j-\frac{1}{2}, k} \\
w_{i, j, k+\frac{1}{2}}
\end{array}\right]=\left[\begin{array}{c}
b_{1} \\
b_{2} \\
b_{3}
\end{array}\right]
$$


Esse sistema pode ser resolvido facilmente pelo método de eliminação de Gauss. Com os valores de $u_{i+\frac{1}{2}, j, k}, v_{i, j-\frac{1}{2}, k}$ e $w_{i, j, k+\frac{1}{2}}$ calculados a pressão é obtida pela discretização de (B.127), no centro da célula de superfície, obtendo,

$$
\begin{aligned}
\tilde{p}_{i, j, k}= & \frac{1}{3 R e}\left[-\left(\frac{u_{i+\frac{1}{2}, j+1, k}+u_{i-\frac{1}{2}, j+1, k}-u_{i+\frac{1}{2}, j, k}-u_{i-\frac{1}{2}, j, k}}{\delta y}\right.\right. \\
& \left.+\frac{v_{i, j+\frac{1}{2}, k}+v_{i, j-\frac{1}{2}, k}-v_{i-1, j+\frac{1}{2}, k}-v_{i-1, j-\frac{1}{2}, k}}{\delta x}\right) \\
& +\left(\frac{u_{i+\frac{1}{2}, j, k}+u_{i-\frac{1}{2}, j, k}-u_{i+\frac{1}{2}, j, k-1}-u_{i-\frac{1}{2}, j, k-1}}{\delta z}\right. \\
& \left.+\frac{w_{i, j, k+\frac{1}{2}}+w_{i, j, k-\frac{1}{2}}-w_{i-1, j, k+\frac{1}{2}}-w_{i-1, j, k-\frac{1}{2}}}{\delta x}\right) \\
& -\left(\frac{v_{i, j+\frac{1}{2}, k}+v_{i, j-\frac{1}{2}, k}-v_{i, j+\frac{1}{2}, k-1}-v_{i, j-\frac{1}{2}, k-1}}{\delta z}\right. \\
& \left.\left.+\frac{w_{i, j+1, k+\frac{1}{2}}+w_{i, j+1, k-\frac{1}{2}}-w_{i, j, k+\frac{1}{2}}-w_{i, j, k-\frac{1}{2}}}{\delta y}\right)\right] \\
& +\frac{1}{3 R e}\left[\Phi_{i, j, k}^{x x}+\Phi_{i, j, k}^{y y}+\Phi_{i, j, k}^{z z}+2\left(-\Phi_{i, j, k}^{x y}+\Phi_{i, j, k}^{x z}-\Phi_{i, j, k}^{y z}\right)\right] .
\end{aligned}
$$

\section{B.3.3 Células $\mathbf{S}$ possuem as faces $\left(i-\frac{1}{2}\right),\left(j-\frac{1}{2}\right)$ e $\left(k+\frac{1}{2}\right)$ em contato com células $\mathrm{E}$.}

$$
\begin{gathered}
\mathbf{n}=\left(-\frac{\sqrt{3}}{3},-\frac{\sqrt{3}}{3}, \frac{\sqrt{3}}{3}\right), \mathbf{m} \mathbf{1}=\left(\frac{\sqrt{2}}{2},-\frac{\sqrt{2}}{2}, 0\right), \mathbf{m} \mathbf{2}=\left(\frac{\sqrt{6}}{6}, \frac{\sqrt{6}}{6}, \frac{\sqrt{6}}{3}\right) . \\
\tilde{p}=\frac{1}{3 R e}\left[2\left(\frac{\partial u}{\partial y}+\frac{\partial v}{\partial x}\right)-2\left(\frac{\partial u}{\partial z}+\frac{\partial w}{\partial x}\right)-2\left(\frac{\partial v}{\partial z}+\frac{\partial w}{\partial y}\right)\right. \\
\left.+\Phi^{x x}+\Phi^{y y}+\Phi^{z z}+2\left(\Phi^{x y}-\Phi^{x z}-\Phi^{y z}\right)\right], \\
-2 \frac{\partial u}{\partial x}+2 \frac{\partial v}{\partial y}+\left(\frac{\partial u}{\partial z}+\frac{\partial w}{\partial x}\right)-\left(\frac{\partial v}{\partial z}+\frac{\partial w}{\partial y}\right)=-\left[-\Phi^{x x}+\Phi^{y y}+\Phi^{x z}-\Phi^{y z}\right], \\
-2 \frac{\partial u}{\partial x}-2 \frac{\partial v}{\partial y}+4 \frac{\partial w}{\partial z}-2\left(\frac{\partial u}{\partial y}+\frac{\partial v}{\partial x}\right)-\left(\frac{\partial u}{\partial z}+\frac{\partial w}{\partial x}\right)-\left(\frac{\partial v}{\partial z}+\frac{\partial w}{\partial y}\right) \\
=-\left[-\Phi^{x x}-\Phi^{y y}+2 \Phi^{z z}-2 \Phi^{x y}-\Phi^{x z}-\Phi^{y z}\right],
\end{gathered}
$$

Agora, adicionando as equações (B.137) e (B.138) obtém-se

$$
-2 \frac{\partial u}{\partial x}+2 \frac{\partial w}{\partial z}-\left(\frac{\partial u}{\partial y}+\frac{\partial v}{\partial x}\right)-\left(\frac{\partial v}{\partial z}+\frac{\partial w}{\partial y}\right)=\left[\Phi^{x x}-\Phi^{z z}+\Phi^{x y}+\Phi^{y z}\right] .
$$

Pela discretização da equação da continuidade obtemos

$$
-u_{i-\frac{1}{2}, j, k}-\frac{\delta x}{\delta y} v_{i, j-\frac{1}{2}, k}+\frac{\delta x}{\delta z} w_{i, j, k+\frac{1}{2}}=b_{3}
$$




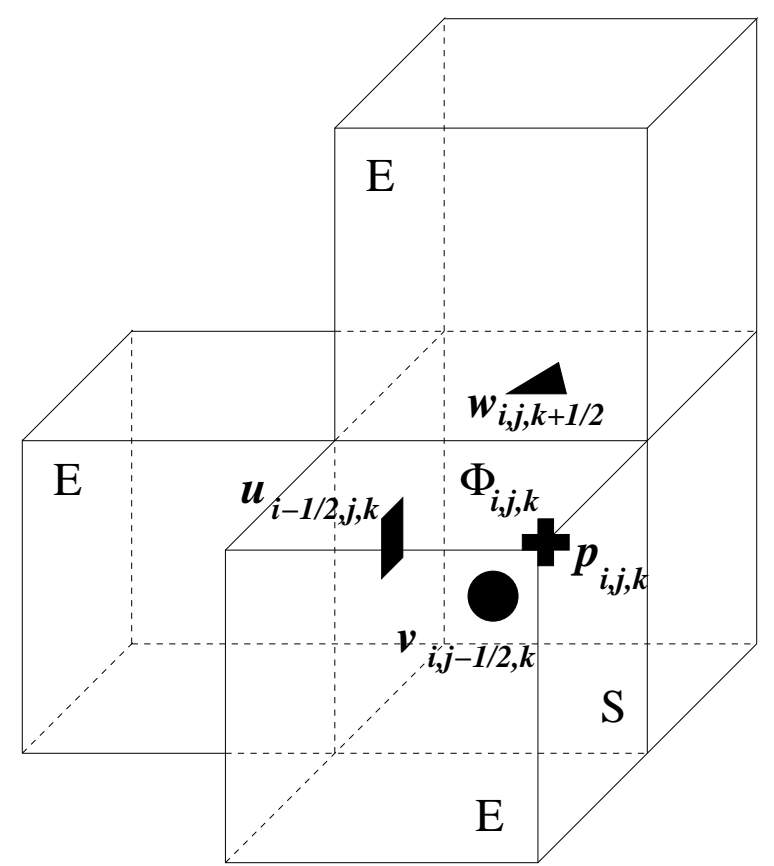

Figura B.19: Célula S com as faces $\left(i-\frac{1}{2}\right),\left(j-\frac{1}{2}\right)$ e $\left(k+\frac{1}{2}\right)$ em contato com faces de células vazias.

onde

$$
b_{3}=-u_{i+\frac{1}{2}, j, k}-\frac{\delta x}{\delta y} v_{i, j+\frac{1}{2}, k}+\frac{\delta x}{\delta z} w_{i, j, k-\frac{1}{2}} .
$$

Agora, equação (B.137) aplicada no centro da célula fornece

$$
\begin{aligned}
& -2\left(\frac{u_{i+\frac{1}{2}, j, k}-u_{i-\frac{1}{2}, j, k}}{\delta x}\right)+2\left(\frac{v_{i, j+\frac{1}{2}, k}-v_{i, j-\frac{1}{2}, k}}{\delta y}\right) \\
& +\frac{1}{2}\left(\frac{u_{i+\frac{1}{2}, j, k}+u_{i-\frac{1}{2}, j, k}-u_{i+\frac{1}{2}, j, k-1}-u_{i-\frac{1}{2}, j, k-1}}{\delta z}\right. \\
& \left.+\frac{w_{i+1, j, k+\frac{1}{2}}+w_{i+1, j, k-\frac{1}{2}}-w_{i, j, k+\frac{1}{2}}-w_{i, j, k-\frac{1}{2}}}{\delta x}\right) \\
& -\frac{1}{2}\left(\frac{v_{i, j+\frac{1}{2}, k}+v_{i, j-\frac{1}{2}, k}-v_{i, j+\frac{1}{2}, k-1}-v_{i, j-\frac{1}{2}, k-1}}{\delta z}\right) \\
& \left.+\frac{w_{i, j+1, k+\frac{1}{2}}+w_{i, j+1, k-\frac{1}{2}}-w_{i, j, k+\frac{1}{2}}-w_{i, j, k-\frac{1}{2}}}{\delta y}\right) \\
& =-\left[-\Phi_{i, j, k}^{x x}+\Phi_{i, j, k}^{y y}+\Phi_{i, j, k}^{x z}-\Phi_{i, j, k}^{y z}\right]
\end{aligned}
$$

que pode ser reescrita como (multiplicando por 2 e por $\delta x$ )

$$
\left(4+\frac{\delta x}{\delta y}\right) u_{i-\frac{1}{2}, j, k}-\left(4 \frac{\delta x}{\delta y}+\frac{\delta x}{\delta z}\right) v_{i, j-\frac{1}{2}, k}+\left(\frac{\delta x}{\delta y}-1\right) w_{i, j, k+\frac{1}{2}}=b_{2}
$$


onde

$$
\begin{aligned}
b_{2}= & 4 u_{i+\frac{1}{2}, j, k}-4 \frac{\delta x}{\delta y} v_{i, j+\frac{1}{2}, k}-\left(\frac{\delta x}{\delta z}\right)\left(u_{i+\frac{1}{2}, j, k}-u_{i+\frac{1}{2}, j, k-1}-u_{i-\frac{1}{2}, j, k-1}\right) \\
& -\left(w_{i+1, j, k+\frac{1}{2}}+w_{i+1, j, k-\frac{1}{2}}-w_{i, j, k-\frac{1}{2}}\right) \\
& +\left(\frac{\delta x}{\delta z}\right)\left(v_{i, j+\frac{1}{2}, k}-v_{i, j+\frac{1}{2}, k-1}-v_{i, j-\frac{1}{2}, k-1}\right) \\
& +\left(\frac{\delta x}{\delta y}\right)\left(w_{i, j+1, k+\frac{1}{2}}+w_{i, j+1, k-\frac{1}{2}}-w_{i, j, k-\frac{1}{2}}\right) \\
& -2 \delta x\left[-\Phi_{i, j, k}^{x x}+\Phi_{i, j, k}^{y y}+\Phi_{i, j, k}^{x z}-\Phi_{i, j, k}^{y z}\right] .
\end{aligned}
$$

Analogamente, discretizando (B.130) da célula de superfície na posição $(i, j, k)$, obtemos

$$
\begin{aligned}
& -2\left(\frac{u_{i+\frac{1}{2}, j, k}-u_{i-\frac{1}{2}, j, k}}{\delta x}\right)+2\left(\frac{w_{i, j, k+\frac{1}{2}}-w_{i, j, k-\frac{1}{2}}}{\delta z}\right) \\
& -\frac{1}{2}\left(\frac{u_{i+\frac{1}{2}, j+1, k}+u_{i-\frac{1}{2}, j+1, k}-u_{i+\frac{1}{2}, j, k}-u_{i-\frac{1}{2}, j, k}}{\delta y}\right. \\
& \left.+\frac{v_{i+1, j+\frac{1}{2}, k}+v_{i+1, j-\frac{1}{2}, k}-v_{i, j+\frac{1}{2}, k}-v_{i, j-\frac{1}{2}, k}}{\delta x}\right) \\
& -\frac{1}{2}\left(\frac{v_{i, j+\frac{1}{2}, k}+v_{i, j-\frac{1}{2}, k}-v_{i, j+\frac{1}{2}, k-1}-v_{i, j-\frac{1}{2}, k-1}}{\delta z}\right. \\
& \left.+\frac{w_{i, j+1, k+\frac{1}{2}}+w_{i, j+1, k-\frac{1}{2}}-w_{i, j, k+\frac{1}{2}}-w_{i, j, k-\frac{1}{2}}}{\delta y}\right) \\
& =\left[\Phi_{i, j, k}^{x x}-\Phi_{i, j, k}^{z z}+\Phi_{i, j, k}^{x y}+\Phi_{i, j, k}^{y z}\right]
\end{aligned}
$$

a qual reescrevemos como

$$
\left(4+\frac{\delta x}{\delta z}\right) u_{i-\frac{1}{2}, j, k}+\left(1-\frac{\delta x}{\delta z}\right) v_{i, j-\frac{1}{2}, k}+\left(4 \frac{\delta x}{\delta z}+\frac{\delta x}{\delta y}\right) w_{i, j, k+\frac{1}{2}}=b_{1}
$$

onde

$$
\begin{aligned}
b_{1}= & 4 u_{i+\frac{1}{2}, j, k}+4 \frac{\delta x}{\delta z} w_{i, j, k-\frac{1}{2}}+\left(\frac{\delta x}{\delta y}\right)\left(u_{i+\frac{1}{2}, j+1, k}+u_{i-\frac{1}{2}, j+1, k}-u_{i+\frac{1}{2}, j, k}\right) \\
& +\left(v_{i+1, j+\frac{1}{2}, k}+v_{i+1, j-\frac{1}{2}, k}-v_{i, j+\frac{1}{2}, k}\right) \\
& +\left(\frac{\delta x}{\delta z}\right)\left(v_{i, j+\frac{1}{2}, k}-v_{i, j+\frac{1}{2}, k-1}-v_{i, j-\frac{1}{2}, k-1}\right) \\
& +\left(\frac{\delta x}{\delta y}\right)\left(w_{i, j+1, k+\frac{1}{2}}+w_{i, j+1, k-\frac{1}{2}}-w_{i, j, k-\frac{1}{2}}\right) \\
& +2 \delta x\left[\Phi_{i, j, k}^{x x}-\Phi_{i, j, k}^{z z}+\Phi_{i, j, k}^{x y}+\Phi_{i, j, k}^{y z}\right] .
\end{aligned}
$$

As equações (B.142), (B.141) e (B.140) fornecem um sistema linear $(3 \times 3)$ para as velocidades $u_{i-\frac{1}{2}, j, k}, v_{i, j-\frac{1}{2}, k}, w_{i, j, k+\frac{1}{2}}$ que pode ser escrito na forma matricial como:

$$
\left[\begin{array}{ccc}
\left(4+\frac{\delta x}{\delta y}\right) & \left(1-\frac{\delta x}{\delta z}\right) & \left(4 \frac{\delta x}{\delta z}+\frac{\delta x}{\delta y}\right) \\
\left(4+\frac{\delta x}{\delta z}\right) & -\left(4 \frac{\delta x}{\delta y}+\frac{\delta x}{\delta z}\right) & \left(\frac{\delta x}{\delta y}-1\right) \\
-1 & -\frac{\delta x}{\delta y} & \frac{\delta x}{\delta z}
\end{array}\right]\left[\begin{array}{c}
u_{i-\frac{1}{2}, j, k} \\
v_{i, j-\frac{1}{2}, k} \\
w_{i, j, k+\frac{1}{2}}
\end{array}\right]=\left[\begin{array}{c}
b_{1} \\
b_{2} \\
b_{3}
\end{array}\right]
$$


Esse sistema pode ser resolvido facilmente pelo método de eliminação de Gauss. Com os valores de $u_{i-\frac{1}{2}, j, k}, v_{i, j-\frac{1}{2}, k}$ e $w_{i, j, k+\frac{1}{2}}$ calculados a pressão é obtida pela discretização de (B.136), no centro da célula de superfície, obtendo,

$$
\begin{aligned}
\tilde{p}_{i, j, k}= & \frac{1}{3 R e}\left[\left(\frac{u_{i+\frac{1}{2}, j+1, k}+u_{i-\frac{1}{2}, j+1, k}-u_{i+\frac{1}{2}, j, k}-u_{i-\frac{1}{2}, j, k}}{\delta y}\right.\right. \\
& \left.+\frac{v_{i+1, j+\frac{1}{2}, k}+v_{i+1, j-\frac{1}{2}, k}-v_{i, j+\frac{1}{2}, k}-v_{i, j-\frac{1}{2}, k}}{\delta x}\right) \\
& -\left(\frac{u_{i+\frac{1}{2}, j, k}+u_{i-\frac{1}{2}, j, k}-u_{i+\frac{1}{2}, j, k-1}-u_{i-\frac{1}{2}, j, k-1}}{\delta z}\right. \\
& \left.+\frac{w_{i+1, j, k+\frac{1}{2}}+w_{i+1, j, k-\frac{1}{2}}-w_{i, j, k+\frac{1}{2}}-w_{i, j, k-\frac{1}{2}}}{\delta x}\right) \\
& -\left(\frac{v_{i, j+\frac{1}{2}, k}+v_{i, j-\frac{1}{2}, k}-v_{i, j+\frac{1}{2}, k-1}-v_{i, j-\frac{1}{2}, k-1}}{\delta z}\right. \\
& \left.\left.+\frac{w_{i, j+1, k+\frac{1}{2}}+w_{i, j+1, k-\frac{1}{2}}-w_{i, j, k+\frac{1}{2}}-w_{i, j, k-\frac{1}{2}}}{\delta y}\right)\right] \\
& +\frac{1}{3 R e}\left[\Phi_{i, j, k}^{x x}+\Phi_{i, j, k}^{y y}+\Phi_{i, j, k}^{z z}+2\left(\Phi_{i, j, k}^{x y}-\Phi_{i, j, k}^{x z}-\Phi_{i, j, k}^{y z}\right)\right] .
\end{aligned}
$$

\section{B.3.4 Células S possuem as faces $\left(i+\frac{1}{2}\right),\left(j+\frac{1}{2}\right)$ e $\left(k-\frac{1}{2}\right)$ em contato com células $\mathrm{E}$.}

$$
\begin{gathered}
\mathbf{n}=\left(\frac{\sqrt{3}}{3}, \frac{\sqrt{3}}{3},-\frac{\sqrt{3}}{3}\right), \mathbf{m} \mathbf{1}=\left(\frac{\sqrt{2}}{2},-\frac{\sqrt{2}}{2}, 0\right), \mathbf{m} \mathbf{2}=\left(\frac{\sqrt{6}}{6}, \frac{\sqrt{6}}{6}, \frac{\sqrt{6}}{3}\right) . \\
\tilde{p}=\frac{1}{3 R e}\left[2\left(\frac{\partial u}{\partial y}+\frac{\partial v}{\partial x}\right)-2\left(\frac{\partial u}{\partial z}+\frac{\partial w}{\partial x}\right)-2\left(\frac{\partial v}{\partial z}+\frac{\partial w}{\partial y}\right)\right. \\
\left.+\Phi^{x x}+\Phi^{y y}+\Phi^{z z}+2\left(\Phi^{x y}-\Phi^{x z}-\Phi^{y z}\right)\right], \\
2 \frac{\partial u}{\partial x}-2 \frac{\partial v}{\partial y}-\left(\frac{\partial u}{\partial z}+\frac{\partial w}{\partial x}\right)+\left(\frac{\partial v}{\partial z}+\frac{\partial w}{\partial y}\right)=-\left[\Phi^{x x}-\Phi^{y y}-\Phi^{x z}+\Phi^{y z}\right], \\
2 \frac{\partial u}{\partial x}+2 \frac{\partial v}{\partial y}-4 \frac{\partial w}{\partial z}+2\left(\frac{\partial u}{\partial y}+\frac{\partial v}{\partial x}\right)+\left(\frac{\partial u}{\partial z}+\frac{\partial w}{\partial x}\right)+\left(\frac{\partial v}{\partial z}+\frac{\partial w}{\partial y}\right) \\
=-\left[\Phi^{x x}+\Phi^{y y}-2 \Phi^{z z}+2 \Phi^{x y}+\Phi^{x z}+\Phi^{y z}\right],
\end{gathered}
$$

Agora, adicionando as equações (B.146) e (B.147) obtém-se

$$
2 \frac{\partial u}{\partial x}-2 \frac{\partial w}{\partial z}+\left(\frac{\partial u}{\partial y}+\frac{\partial v}{\partial x}\right)+\left(\frac{\partial v}{\partial z}+\frac{\partial w}{\partial y}\right)=\left[-\Phi^{x x}+\Phi^{z z}-\Phi^{x y}-\Phi^{y z}\right] .
$$

Pela discretização da equação da continuidade obtemos

$$
u_{i+\frac{1}{2}, j, k}+\frac{\delta x}{\delta y} v_{i, j+\frac{1}{2}, k}-\frac{\delta x}{\delta z} w_{i, j, k-\frac{1}{2}}=b_{3}
$$




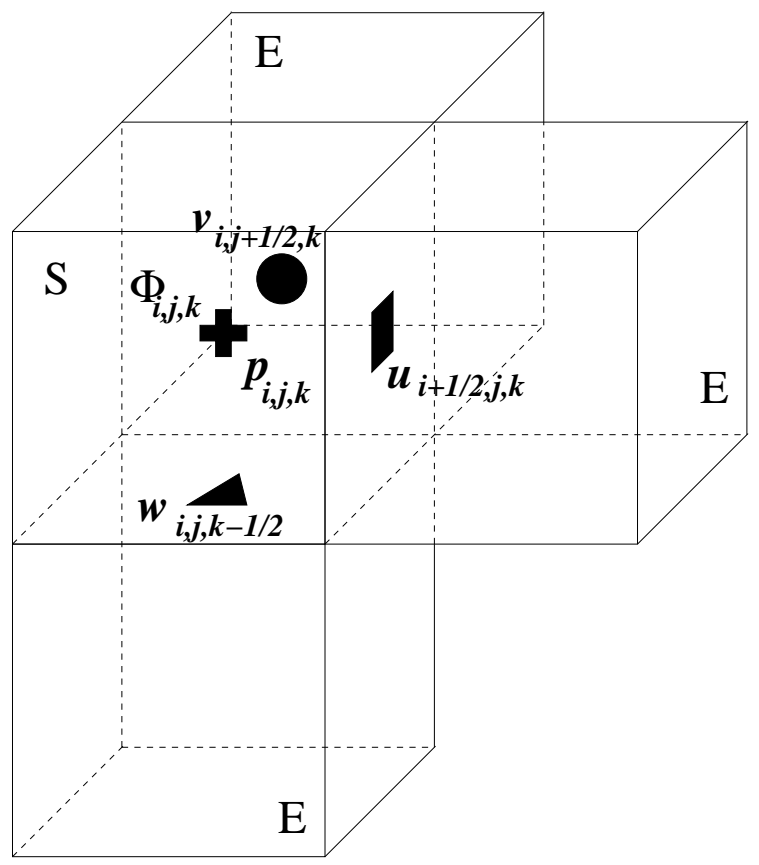

Figura B.20: Célula S com as faces $\left(i+\frac{1}{2}\right),\left(j+\frac{1}{2}\right)$ e $\left(k-\frac{1}{2}\right)$ em contato com faces de células vazias.

onde

$$
b_{3}=u_{i-\frac{1}{2}, j, k}+\frac{\delta x}{\delta y} v_{i, j-\frac{1}{2}, k}-\frac{\delta x}{\delta z} w_{i, j, k+\frac{1}{2}} .
$$

Agora, equação (B.146) aplicada no centro da célula fornece

$$
\begin{aligned}
& 2\left(\frac{u_{i+\frac{1}{2}, j, k}-u_{i-\frac{1}{2}, j, k}}{\delta x}\right)-2\left(\frac{v_{i, j+\frac{1}{2}, k}-v_{i, j-\frac{1}{2}, k}}{\delta y}\right) \\
& -\frac{1}{2}\left(\frac{u_{i+\frac{1}{2}, j, k+1}+u_{i-\frac{1}{2}, j, k+1}-u_{i+\frac{1}{2}, j, k}-u_{i-\frac{1}{2}, j, k}}{\delta z}\right. \\
& \left.+\frac{w_{i, j, k+\frac{1}{2}}+w_{i, j, k-\frac{1}{2}}-w_{i-1, j, k+\frac{1}{2}}-w_{i-1, j, k-\frac{1}{2}}}{\delta x}\right) \\
& +\frac{1}{2}\left(\frac{v_{i, j+\frac{1}{2}, k+1}+v_{i, j-\frac{1}{2}, k+1}-v_{i, j+\frac{1}{2}, k}-v_{i, j-\frac{1}{2}, k}}{\delta z}\right. \\
& \left.+\frac{w_{i, j, k+\frac{1}{2}}+w_{i, j, k-\frac{1}{2}}-w_{i, j-1, k+\frac{1}{2}}-w_{i, j-1, k-\frac{1}{2}}}{\delta y}\right) \\
& =-\left[\Phi_{i, j, k}^{x x}-\Phi_{i, j, k}^{y y}-\Phi_{i, j, k}^{x z}+\Phi_{i, j, k}^{y z}\right]
\end{aligned}
$$

que pode ser reescrita como (multiplicando por 2 e por $\delta x$ )

$$
\left(4+\frac{\delta x}{\delta z}\right) u_{i+\frac{1}{2}, j, k}-\left(4 \frac{\delta x}{\delta y}+\frac{\delta x}{\delta z}\right) v_{i, j+\frac{1}{2}, k}+\left(\frac{\delta x}{\delta y}-1\right) w_{i, j, k-\frac{1}{2}}=b_{2}
$$


onde

$$
\begin{aligned}
b_{2}= & 4 u_{i-\frac{1}{2}, j, k}-4 \frac{\delta x}{\delta y} v_{i, j-\frac{1}{2}, k}+\left(\frac{\delta x}{\delta z}\right)\left(u_{i+\frac{1}{2}, j, k+1}+u_{i-\frac{1}{2}, j, k+1}-u_{i-\frac{1}{2}, j, k}\right) \\
& +\left(w_{i, j, k+\frac{1}{2}}-w_{i-1, j, k+\frac{1}{2}}-w_{i-1, j, k-\frac{1}{2}}\right) \\
& -\left(\frac{\delta x}{\delta z}\right)\left(v_{i, j+\frac{1}{2}, k+1}+v_{i, j-\frac{1}{2}, k+1}-v_{i, j-\frac{1}{2}, k}\right) \\
& -\left(\frac{\delta x}{\delta y}\right)\left(w_{i, j, k+\frac{1}{2}}-w_{i, j-1, k+\frac{1}{2}}-w_{i, j-1, k-\frac{1}{2}}\right) \\
& -2 \delta x\left[\Phi_{i, j, k}^{x x}-\Phi_{i, j, k}^{y y}-\Phi_{i, j, k}^{x z}+\Phi_{i, j, k}^{y z}\right] .
\end{aligned}
$$

Analogamente, discretizando (B.148) da célula de superfície na posição $(i, j, k)$, obtemos

$$
\begin{aligned}
& 2\left(\frac{u_{i+\frac{1}{2}, j, k}-u_{i-\frac{1}{2}, j, k}}{\delta x}\right)-2\left(\frac{w_{i, j, k+\frac{1}{2}}-w_{i, j, k-\frac{1}{2}}}{\delta z}\right) \\
& +\frac{1}{2}\left(\frac{u_{i+\frac{1}{2}, j, k}+u_{i-\frac{1}{2}, j, k}-u_{i+\frac{1}{2}, j-1, k}-u_{i-\frac{1}{2}, j-1, k}}{\delta y}\right. \\
& \left.+\frac{v_{i, j+\frac{1}{2}, k}+v_{i, j-\frac{1}{2}, k}-v_{i-1, j+\frac{1}{2}, k}-v_{i-1, j-\frac{1}{2}, k}}{\delta x}\right) \\
& +\frac{1}{2}\left(\frac{v_{i, j+\frac{1}{2}, k+1}+v_{i, j-\frac{1}{2}, k+1}-v_{i, j+\frac{1}{2}, k}-v_{i, j-\frac{1}{2}, k}}{\delta z}\right) \\
& \left.+\frac{w_{i, j, k+\frac{1}{2}}+w_{i, j, k-\frac{1}{2}}-w_{i, j-1, k+\frac{1}{2}}-w_{i, j-1, k-\frac{1}{2}}}{\delta y}\right) \\
& =\left[-\Phi_{i, j, k}^{x x}+\Phi_{i, j, k}^{z z}-\Phi_{i, j, k}^{x y}-\Phi_{i, j, k}^{y z}\right]
\end{aligned}
$$

a qual reescrevemos como

$$
\left(4+\frac{\delta x}{\delta y}\right) u_{i+\frac{1}{2}, j, k}+\left(1-\frac{\delta x}{\delta z}\right) v_{i, j+\frac{1}{2}, k}+\left(4 \frac{\delta x}{\delta z}+\frac{\delta x}{\delta y}\right) w_{i, j, k-\frac{1}{2}}=b_{1}
$$

onde

$$
\begin{aligned}
b_{1}= & 4 u_{i-\frac{1}{2}, j, k}+4 \frac{\delta x}{\delta z} w_{i, j, k+\frac{1}{2}}-\left(\frac{\delta x}{\delta y}\right)\left(u_{i-\frac{1}{2}, j, k}-u_{i+\frac{1}{2}, j-1, k}-u_{i-\frac{1}{2}, j-1, k}\right) \\
& -\left(v_{i, j-\frac{1}{2}, k}-v_{i-1, j+\frac{1}{2}, k}-v_{i-1, j-\frac{1}{2}, k}\right) \\
& -\left(\frac{\delta x}{\delta z}\right)\left(v_{i, j+\frac{1}{2}, k+1}+v_{i, j-\frac{1}{2}, k+1}-v_{i, j-\frac{1}{2}, k}\right) \\
& -\left(\frac{\delta x}{\delta y}\right)\left(w_{i, j, k+\frac{1}{2}}-w_{i, j-1, k+\frac{1}{2}}-w_{i, j-1, k-\frac{1}{2}}\right) \\
& +2 \delta x\left[-\Phi_{i, j, k}^{x x}+\Phi_{i, j, k}^{z z}-\Phi_{i, j, k}^{x y}-\Phi_{i, j, k}^{y z}\right] .
\end{aligned}
$$

As equações (B.151), (B.150) e (B.149) fornecem um sistema linear $(3 \times 3)$ para as velocidades $u_{i+\frac{1}{2}, j, k}, v_{i, j+\frac{1}{2}, k}, w_{i, j, k-\frac{1}{2}}$ que pode ser escrito na forma matricial como:

$$
\left[\begin{array}{ccc}
\left(4+\frac{\delta x}{\delta y}\right) & \left(1-\frac{\delta x}{\delta z}\right) & \left(4 \frac{\delta x}{\delta z}+\frac{\delta x}{\delta y}\right) \\
\left(4+\frac{\delta x}{\delta z}\right) & -\left(4 \frac{\delta x}{\delta y}+\frac{\delta x}{\delta z}\right) & \left(\frac{\delta x}{\delta y}-1\right) \\
1 & \frac{\delta x}{\delta y} & -\frac{\delta x}{\delta z}
\end{array}\right]\left[\begin{array}{c}
u_{i+\frac{1}{2}, j, k} \\
v_{i, j+\frac{1}{2}, k} \\
w_{i, j, k-\frac{1}{2}}
\end{array}\right]=\left[\begin{array}{c}
b_{1} \\
b_{2} \\
b_{3}
\end{array}\right]
$$


Esse sistema pode ser resolvido facilmente pelo método de eliminação de Gauss. Com os valores de $u_{i+\frac{1}{2}, j, k}, v_{i, j+\frac{1}{2}, k}$ e $w_{i, j, k-\frac{1}{2}}$ calculados a pressão é obtida pela discretização de (B.145), no centro da célula de superfície, obtendo,

$$
\begin{aligned}
\tilde{p}_{i, j, k}= & \frac{1}{3 R e}\left[\left(\frac{u_{i+\frac{1}{2}, j, k}+u_{i-\frac{1}{2}, j, k}-u_{i+\frac{1}{2}, j-1, k}-u_{i-\frac{1}{2}, j-1, k}}{\delta y}\right.\right. \\
& \left.+\frac{v_{i, j+\frac{1}{2}, k}+v_{i, j-\frac{1}{2}, k}-v_{i-1, j+\frac{1}{2}, k}-v_{i-1, j-\frac{1}{2}, k}}{\delta x}\right) \\
& -\left(\frac{u_{i+\frac{1}{2}, j, k+1}+u_{i-\frac{1}{2}, j, k+1}-u_{i+\frac{1}{2}, j, k}-u_{i-\frac{1}{2}, j, k}}{\delta z}\right. \\
& \left.+\frac{w_{i, j, k+\frac{1}{2}}+w_{i, j, k-\frac{1}{2}}-w_{i-1, j, k+\frac{1}{2}}-w_{i-1, j, k-\frac{1}{2}}}{\delta x}\right) \\
& -\left(\frac{v_{i, j+\frac{1}{2}, k+1}+v_{i, j-\frac{1}{2}, k+1}-v_{i, j+\frac{1}{2}, k}-v_{i, j-\frac{1}{2}, k}}{\delta z}\right. \\
& \left.\left.+\frac{w_{i, j, k+\frac{1}{2}}+w_{i, j, k-\frac{1}{2}}-w_{i, j-1, k+\frac{1}{2}}-w_{i, j-1, k-\frac{1}{2}}}{\delta y}\right)\right] \\
& +\frac{1}{3 R e}\left[\Phi_{i, j, k}^{x x}+\Phi_{i, j, k}^{y y}+\Phi_{i, j, k}^{z z}+2\left(\Phi_{i, j, k}^{x y}-\Phi_{i, j, k}^{x z}-\Phi_{i, j, k}^{y z}\right)\right] .
\end{aligned}
$$

\section{B.3.5 Células $\mathbf{S}$ possuem as faces $\left(i-\frac{1}{2}\right),\left(j+\frac{1}{2}\right)$ e $\left(k-\frac{1}{2}\right)$ em contato com células $\mathrm{E}$.}

$$
\begin{gathered}
\mathbf{n}=\left(-\frac{\sqrt{3}}{3}, \frac{\sqrt{3}}{3},-\frac{\sqrt{3}}{3}\right), \mathbf{m} \mathbf{1}=\left(\frac{\sqrt{2}}{2}, 0,-\frac{\sqrt{2}}{2}\right), \mathbf{m} \mathbf{2}=\left(\frac{\sqrt{6}}{6}, \frac{\sqrt{6}}{3}, \frac{\sqrt{6}}{6}\right) . \\
\tilde{p}=\frac{1}{3 R e}\left[-2\left(\frac{\partial u}{\partial y}+\frac{\partial v}{\partial x}\right)+2\left(\frac{\partial u}{\partial z}+\frac{\partial w}{\partial x}\right)-2\left(\frac{\partial v}{\partial z}+\frac{\partial w}{\partial y}\right)\right. \\
\left.+\Phi^{x x}+\Phi^{y y}+\Phi^{z z}+2\left(-\Phi^{x y}+\Phi^{x z}-\Phi^{y z}\right)\right], \\
-2 \frac{\partial u}{\partial x}+2 \frac{\partial w}{\partial z}+\left(\frac{\partial u}{\partial y}+\frac{\partial v}{\partial x}\right)-\left(\frac{\partial v}{\partial z}+\frac{\partial w}{\partial y}\right)=-\left[-\Phi^{x x}+\Phi^{z z}+\Phi^{x y}-\Phi^{y z}\right] \\
-2 \frac{\partial u}{\partial x}+4 \frac{\partial v}{\partial y}-2 \frac{\partial w}{\partial z}-\left(\frac{\partial u}{\partial y}+\frac{\partial v}{\partial x}\right)-2\left(\frac{\partial u}{\partial z}+\frac{\partial w}{\partial x}\right)-\left(\frac{\partial v}{\partial z}+\frac{\partial w}{\partial y}\right) \\
=-\left[-\Phi^{x x}+2 \Phi^{y y}-\Phi^{z z}-\Phi^{x y}-2 \Phi^{x z}-\Phi^{y z}\right],
\end{gathered}
$$

Agora, adicionando as equações (B.155) e (B.156) obtém-se

$$
-2 \frac{\partial u}{\partial x}+2 \frac{\partial v}{\partial y}-\left(\frac{\partial u}{\partial z}+\frac{\partial w}{\partial x}\right)-\left(\frac{\partial v}{\partial z}+\frac{\partial w}{\partial y}\right)=\left[\Phi^{x x}-\Phi^{y y}+\Phi^{x z}+\Phi^{y z}\right] .
$$

Pela discretização da equação da continuidade obtemos

$$
-u_{i-\frac{1}{2}, j, k}+\frac{\delta x}{\delta y} v_{i, j+\frac{1}{2}, k}-\frac{\delta x}{\delta z} w_{i, j, k-\frac{1}{2}}=b_{3}
$$




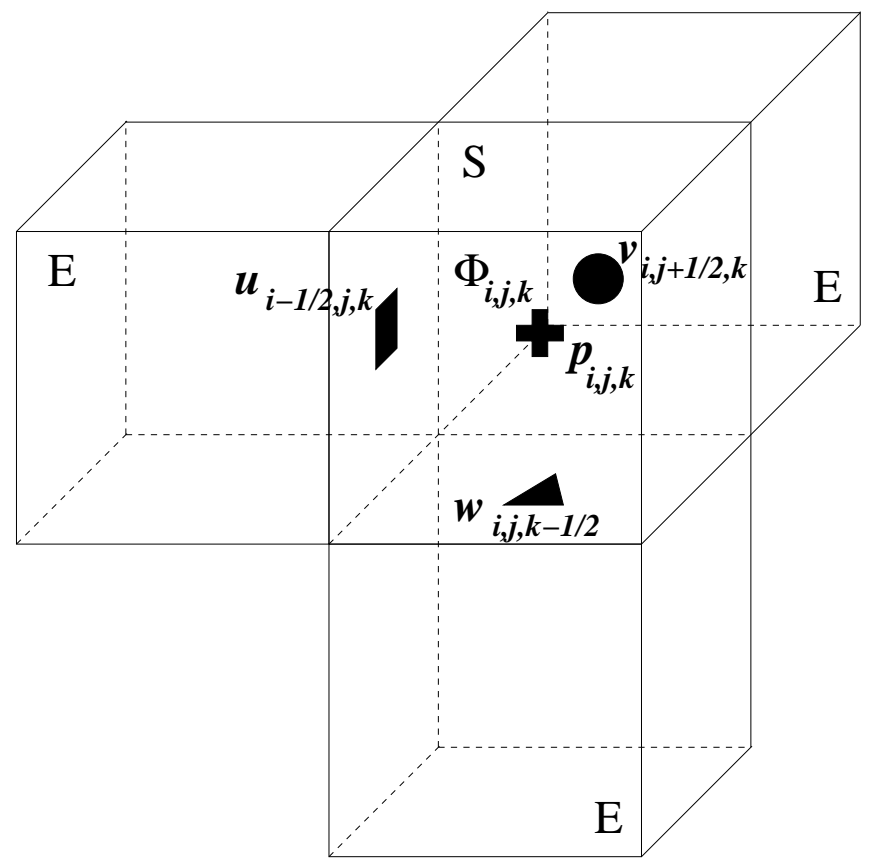

Figura B.21: Célula S com as faces $\left(i-\frac{1}{2}\right),\left(j+\frac{1}{2}\right)$ e $\left(k-\frac{1}{2}\right)$ em contato com faces de células vazias.

onde

$$
b_{3}=-u_{i+\frac{1}{2}, j, k}+\frac{\delta x}{\delta y} v_{i, j-\frac{1}{2}, k}-\frac{\delta x}{\delta z} w_{i, j, k+\frac{1}{2}} .
$$

Agora, equação (B.155) aplicada no centro da célula fornece

$$
\begin{aligned}
& -2\left(\frac{u_{i+\frac{1}{2}, j, k}-u_{i-\frac{1}{2}, j, k}}{\delta x}\right)+2\left(\frac{w_{i, j, k+\frac{1}{2}}-w_{i, j, k-\frac{1}{2}}}{\delta z}\right) \\
& +\frac{1}{2}\left(\frac{u_{i+\frac{1}{2}, j, k}+u_{i-\frac{1}{2}, j, k}-u_{i+\frac{1}{2}, j-1, k}-u_{i-\frac{1}{2}, j-1, k}}{\delta y}\right. \\
& \left.+\frac{v_{i+1, j+\frac{1}{2}, k}+v_{i+1, j-\frac{1}{2}, k}-v_{i, j+\frac{1}{2}, k}-v_{i, j-\frac{1}{2}, k}}{\delta x}\right) \\
& -\frac{1}{2}\left(\frac{v_{i, j+\frac{1}{2}, k+1}+v_{i, j-\frac{1}{2}, k+1}-v_{i, j+\frac{1}{2}, k}-v_{i, j-\frac{1}{2}, k}}{\delta z}\right. \\
& \left.+\frac{w_{i, j, k+\frac{1}{2}}+w_{i, j, k-\frac{1}{2}}-w_{i, j-1, k+\frac{1}{2}}-w_{i, j-1, k-\frac{1}{2}}}{\delta y}\right) \\
& =-\left[-\Phi_{i, j, k}^{x x}+\Phi_{i, j, k}^{z z}+\Phi_{i, j, k}^{x y}-\Phi_{i, j, k}^{y z}\right]
\end{aligned}
$$

que pode ser reescrita como (multiplicando por 2 e por $\delta x$ )

$$
\left(4+\frac{\delta x}{\delta y}\right) u_{i-\frac{1}{2}, j, k}+\left(\frac{\delta x}{\delta z}-1\right) v_{i, j+\frac{1}{2}, k}-\left(4 \frac{\delta x}{\delta z}+\frac{\delta x}{\delta y}\right) w_{i, j, k-\frac{1}{2}}=b_{2}
$$


onde

$$
\begin{aligned}
b_{2}= & 4 u_{i+\frac{1}{2}, j, k}-4 \frac{\delta x}{\delta z} w_{i, j, k+\frac{1}{2}}-\left(\frac{\delta x}{\delta y}\right)\left(u_{i+\frac{1}{2}, j, k}-u_{i+\frac{1}{2}, j-1, k}-u_{i-\frac{1}{2}, j-1, k}\right) \\
& -\left(v_{i+1, j+\frac{1}{2}, k}+v_{i+1, j-\frac{1}{2}, k}-v_{i, j-\frac{1}{2}, k}\right) \\
& +\left(\frac{\delta x}{\delta z}\right)\left(v_{i, j+\frac{1}{2}, k+1}+v_{i, j-\frac{1}{2}, k+1}-v_{i, j-\frac{1}{2}, k}\right) \\
& +\left(\frac{\delta x}{\delta y}\right)\left(w_{i, j, k+\frac{1}{2}}-w_{i, j-1, k+\frac{1}{2}}-w_{i, j-1, k-\frac{1}{2}}\right) \\
& -2 \delta x\left[-\Phi_{i, j, k}^{x x}+\Phi_{i, j, k}^{z z}+\Phi_{i, j, k}^{x y}-\Phi_{i, j, k}^{y z}\right] .
\end{aligned}
$$

Analogamente, discretizando (B.157) da célula de superfície na posição $(i, j, k)$, obtemos

$$
\begin{aligned}
& -2\left(\frac{u_{i+\frac{1}{2}, j, k}-u_{i-\frac{1}{2}, j, k}}{\delta x}\right)+2\left(\frac{v_{i, j+\frac{1}{2}, k}-v_{i, j-\frac{1}{2}, k}}{\delta y}\right) \\
& -\frac{1}{2}\left(\frac{u_{i+\frac{1}{2}, j, k+1}+u_{i-\frac{1}{2}, j, k+1}-u_{i+\frac{1}{2}, j, k}-u_{i-\frac{1}{2}, j, k}}{\delta z}\right. \\
& \left.+\frac{w_{i+1, j+\frac{1}{2}, k}+w_{i+1, j-\frac{1}{2}, k}-w_{i, j+\frac{1}{2}, k}-w_{i, j-\frac{1}{2}, k}}{\delta x}\right) \\
& -\frac{1}{2}\left(\frac{v_{i, j+\frac{1}{2}, k+1}+v_{i, j-\frac{1}{2}, k+1}-v_{i, j+\frac{1}{2}, k}-v_{i, j-\frac{1}{2}, k}}{\delta z}\right) \\
& \left.+\frac{w_{i, j, k+\frac{1}{2}}+w_{i, j, k-\frac{1}{2}}-w_{i, j-1, k+\frac{1}{2}}-w_{i, j-1, k-\frac{1}{2}}}{\delta y}\right) \\
& =\left[\Phi_{i, j, k}^{x x}-\Phi_{i, j, k}^{y y}+\Phi_{i, j, k}^{x z}+\Phi_{i, j, k}^{y z}\right]
\end{aligned}
$$

a qual reescrevemos como

$$
\left(4+\frac{\delta x}{\delta z}\right) u_{i-\frac{1}{2}, j, k}+\left(4 \frac{\delta x}{\delta y}+\frac{\delta x}{\delta z}\right) v_{i, j+\frac{1}{2}, k}+\left(1-\frac{\delta x}{\delta y}\right) w_{i, j, k-\frac{1}{2}}=b_{1}
$$

onde

$$
\begin{aligned}
b_{1}= & 4 u_{i+\frac{1}{2}, j, k}+4 \frac{\delta x}{\delta y} v_{i, j-\frac{1}{2}, k}+\left(\frac{\delta x}{\delta z}\right)\left(u_{i+\frac{1}{2}, j, k+1}+u_{i-\frac{1}{2}, j, k+1}-u_{i+\frac{1}{2}, j, k}\right) \\
& +\left(w_{i+1, j, k+\frac{1}{2}}+w_{i+1, j, k-\frac{1}{2}}-w_{i, j, k+\frac{1}{2}}\right) \\
& +\left(\frac{\delta x}{\delta z}\right)\left(v_{i, j+\frac{1}{2}, k+1}+v_{i, j-\frac{1}{2}, k+1}-v_{i, j-\frac{1}{2}, k}\right) \\
& +\left(\frac{\delta x}{\delta y}\right)\left(w_{i, j, k+\frac{1}{2}}-w_{i, j-1, k+\frac{1}{2}}-w_{i, j-1, k-\frac{1}{2}}\right) \\
& +2 \delta x\left[\Phi_{i, j, k}^{x x}-\Phi_{i, j, k}^{y y}+\Phi_{i, j, k}^{x z}+\Phi_{i, j, k}^{y z}\right] .
\end{aligned}
$$

As equações (B.160), (B.159) e (B.158) fornecem um sistema linear $(3 \times 3)$ para as velocidades $u_{i-\frac{1}{2}, j, k}, v_{i, j+\frac{1}{2}, k}, w_{i, j, k-\frac{1}{2}}$ que pode ser escrito na forma matricial como:

$$
\left[\begin{array}{ccc}
\left(4+\frac{\delta x}{\delta z}\right) & \left(4 \frac{\delta x}{\delta y}+\frac{\delta x}{\delta z}\right) & \left(1-\frac{\delta x}{\delta y}\right) \\
\left(4+\frac{\delta x}{\delta y}\right) & \left(\frac{\delta x}{\delta z}-1\right) & -\left(4 \frac{\delta x}{\delta z}+\frac{\delta x}{\delta y}\right) \\
-1 & \frac{\delta x}{\delta y} & -\frac{\delta x}{\delta z}
\end{array}\right]\left[\begin{array}{c}
u_{i-\frac{1}{2}, j, k} \\
v_{i, j+\frac{1}{2}, k} \\
w_{i, j, k-\frac{1}{2}}
\end{array}\right]=\left[\begin{array}{c}
b_{1} \\
b_{2} \\
b_{3}
\end{array}\right]
$$


Esse sistema pode ser resolvido facilmente pelo método de eliminação de Gauss. Com os valores de $u_{i-\frac{1}{2}, j, k}, v_{i, j+\frac{1}{2}, k}$ e $w_{i, j, k-\frac{1}{2}}$ calculados a pressão é obtida pela discretização de (B.154), no centro da célula de superfície, obtendo,

$$
\begin{aligned}
\tilde{p}_{i, j, k}= & \frac{1}{3 R e}\left[-\left(\frac{u_{i+\frac{1}{2}, j, k}+u_{i-\frac{1}{2}, j, k}-u_{i+\frac{1}{2}, j-1, k}-u_{i-\frac{1}{2}, j-1, k}}{\delta y}\right.\right. \\
& \left.+\frac{v_{i+1, j+\frac{1}{2}, k}+v_{i+1, j-\frac{1}{2}, k}-v_{i, j+\frac{1}{2}, k}-v_{i, j-\frac{1}{2}, k}}{\delta x}\right) \\
& +\left(\frac{u_{i+\frac{1}{2}, j, k+1}+u_{i-\frac{1}{2}, j, k+1}-u_{i+\frac{1}{2}, j, k}-u_{i-\frac{1}{2}, j, k}}{\delta z}\right. \\
& \left.+\frac{w_{i+1, j, k+\frac{1}{2}}+w_{i+1, j, k-\frac{1}{2}}-w_{i, j, k+\frac{1}{2}}-w_{i, j, k-\frac{1}{2}}}{\delta x}\right) \\
& -\left(\frac{v_{i, j+\frac{1}{2}, k+1}+v_{i, j-\frac{1}{2}, k+1}-v_{i, j+\frac{1}{2}, k}-v_{i, j-\frac{1}{2}, k}}{\delta z}\right. \\
& \left.\left.+\frac{w_{i, j, k+\frac{1}{2}}+w_{i, j, k-\frac{1}{2}}-w_{i, j-1, k+\frac{1}{2}}-w_{i, j-1, k-\frac{1}{2}}}{\delta y}\right)\right] \\
& +\frac{1}{3 R e}\left[\Phi_{i, j, k}^{x x}+\Phi_{i, j, k}^{y y}+\Phi_{i, j, k}^{z z}+2\left(-\Phi_{i, j, k}^{x y}+\Phi_{i, j, k}^{x z}-\Phi_{i, j, k}^{y z}\right)\right] .
\end{aligned}
$$

\section{B.3.6 Células S possuem as faces $\left(i+\frac{1}{2}\right),\left(j-\frac{1}{2}\right)$ e $\left(k-\frac{1}{2}\right)$ em contato com células $\mathrm{E}$.}

$$
\mathbf{n}=\left(\frac{\sqrt{3}}{3},-\frac{\sqrt{3}}{3},-\frac{\sqrt{3}}{3}\right), \mathbf{m} \mathbf{1}=\left(0, \frac{\sqrt{2}}{2},-\frac{\sqrt{2}}{2}\right), \mathbf{m} \mathbf{2}=\left(\frac{\sqrt{6}}{3}, \frac{\sqrt{6}}{6}, \frac{\sqrt{6}}{6}\right)
$$

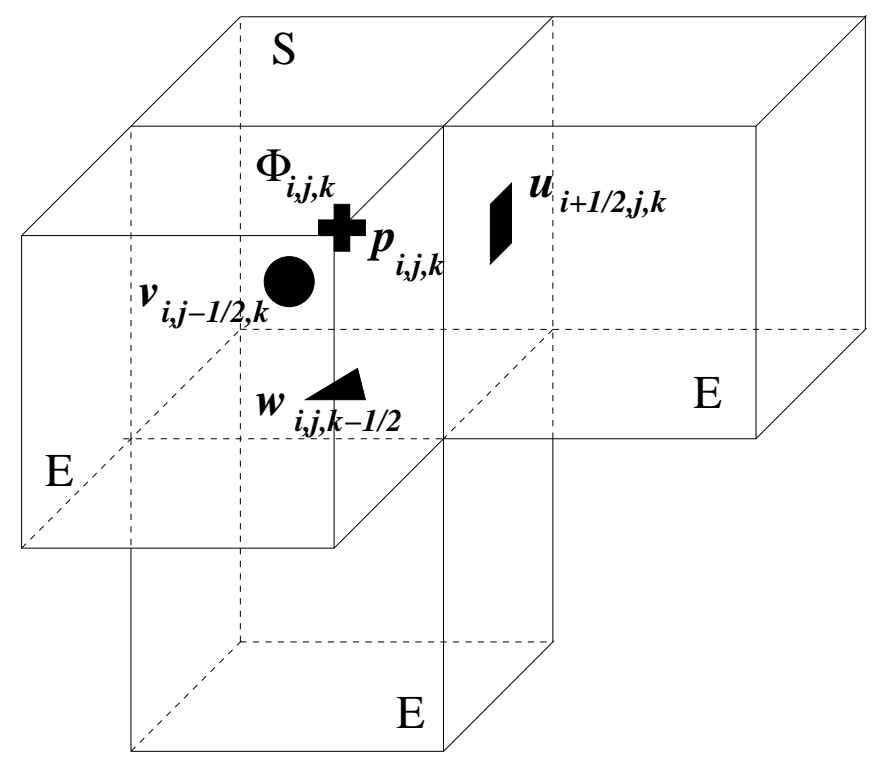

Figura B.22: Célula S com as faces $\left(i+\frac{1}{2}\right),\left(j-\frac{1}{2}\right)$ e $\left(k-\frac{1}{2}\right)$ em contato com faces de células vazias. 


$$
\begin{gathered}
\tilde{p}=\frac{1}{3 R e}\left[-2\left(\frac{\partial u}{\partial y}+\frac{\partial v}{\partial x}\right)-2\left(\frac{\partial u}{\partial z}+\frac{\partial w}{\partial x}\right)+2\left(\frac{\partial v}{\partial z}+\frac{\partial w}{\partial y}\right)\right. \\
\left.+\Phi^{x x}+\Phi^{y y}+\Phi^{z z}+2\left(-\Phi^{x y}-\Phi^{x z}+\Phi^{y z}\right)\right] \\
-2 \frac{\partial v}{\partial y}+2 \frac{\partial w}{\partial z}+\left(\frac{\partial u}{\partial y}+\frac{\partial v}{\partial x}\right)-\left(\frac{\partial u}{\partial z}+\frac{\partial w}{\partial x}\right)=-\left[-\Phi^{y y}+\Phi^{z z}+\Phi^{x y}-\Phi^{x z}\right] \\
4 \frac{\partial u}{\partial x}-2 \frac{\partial v}{\partial y}-2 \frac{\partial w}{\partial z}-\left(\frac{\partial u}{\partial y}+\frac{\partial v}{\partial x}\right)-\left(\frac{\partial u}{\partial z}+\frac{\partial w}{\partial x}\right)-2\left(\frac{\partial v}{\partial z}+\frac{\partial w}{\partial y}\right) \\
=-\left[2 \Phi^{x x}-\Phi^{y y}-\Phi^{z z}-\Phi^{x y}-\Phi^{x z}-2 \Phi^{y z}\right]
\end{gathered}
$$

Agora, adicionando as equações (B.164) e (B.165) obtém-se

$$
2 \frac{\partial u}{\partial x}-2 \frac{\partial v}{\partial y}-\left(\frac{\partial u}{\partial z}+\frac{\partial w}{\partial x}\right)-\left(\frac{\partial v}{\partial z}+\frac{\partial w}{\partial y}\right)=\left[-\Phi^{x x}+\Phi^{y y}+\Phi^{x z}+\Phi^{y z}\right] .
$$

Pela discretização da equação da continuidade obtemos

$$
u_{i+\frac{1}{2}, j, k}-\frac{\delta x}{\delta y} v_{i, j-\frac{1}{2}, k}-\frac{\delta x}{\delta z} w_{i, j, k-\frac{1}{2}}=b_{3}
$$

onde

$$
b_{3}=u_{i-\frac{1}{2}, j, k}-\frac{\delta x}{\delta y} v_{i, j+\frac{1}{2}, k}-\frac{\delta x}{\delta z} w_{i, j, k+\frac{1}{2}} .
$$

Agora, equação (B.164) aplicada no centro da célula fornece

$$
\begin{aligned}
& -2\left(\frac{v_{i, j+\frac{1}{2}, k}-v_{i, j-\frac{1}{2}, k}}{\delta y}\right)+2\left(\frac{w_{i, j, k+\frac{1}{2}}-w_{i, j, k-\frac{1}{2}}}{\delta z}\right) \\
& +\frac{1}{2}\left(\frac{u_{i+\frac{1}{2}, j+1, k}+u_{i-\frac{1}{2}, j+1, k}-u_{i+\frac{1}{2}, j, k}-u_{i-\frac{1}{2}, j, k}}{\delta y}\right. \\
& \left.+\frac{v_{i, j+\frac{1}{2}, k}+v_{i, j-\frac{1}{2}, k}-v_{i-1, j+\frac{1}{2}, k}-v_{i-1, j-\frac{1}{2}, k}}{\delta x}\right) \\
& -\frac{1}{2}\left(\frac{u_{i+\frac{1}{2}, j, k+1}+u_{i-\frac{1}{2}, j, k+1}-u_{i+\frac{1}{2}, j, k}-u_{i-\frac{1}{2}, j, k}}{\delta z}\right. \\
& \left.+\frac{w_{i, j, k+\frac{1}{2}}+w_{i, j, k-\frac{1}{2}}-w_{i-1, j, k+\frac{1}{2}}-w_{i-1, j, k-\frac{1}{2}}}{\delta x}\right) \\
& =-\left[-\Phi_{i, j, k}^{y y}+\Phi_{i, j, k}^{z z}+\Phi_{i, j, k}^{x y}-\Phi_{i, j, k}^{x z}\right]
\end{aligned}
$$

que pode ser reescrita como (multiplicando por 2 e por $\delta x$ )

$$
\left(\frac{\delta x}{\delta z}-\frac{\delta x}{\delta y}\right) u_{i+\frac{1}{2}, j, k}+\left(4 \frac{\delta x}{\delta y}+1\right) v_{i, j-\frac{1}{2}, k}-\left(4 \frac{\delta x}{\delta z}+1\right) w_{i, j, k-\frac{1}{2}}=b_{2}
$$


onde

$$
\begin{aligned}
b_{2}= & 4 \frac{\delta x}{\delta y} v_{i, j+\frac{1}{2}, k}-4 \frac{\delta x}{\delta z} w_{i, j, k+\frac{1}{2}}-\left(\frac{\delta x}{\delta y}\right)\left(u_{i+\frac{1}{2}, j+1, k}+u_{i-\frac{1}{2}, j+1, k}-u_{i-\frac{1}{2}, j, k}\right) \\
& -\left(v_{i, j+\frac{1}{2}, k}-v_{i-1, j+\frac{1}{2}, k}-v_{i-1, j-\frac{1}{2}, k}\right) \\
& +\left(\frac{\delta x}{\delta z}\right)\left(u_{i+\frac{1}{2}, j, k+1}+u_{i-\frac{1}{2}, j, k+1}-u_{i-\frac{1}{2}, j, k}\right) \\
& +\left(w_{i, j, k+\frac{1}{2}}-w_{i-1, j, k+\frac{1}{2}}-w_{i-1, j, k-\frac{1}{2}}\right) \\
& -2 \delta x\left[-\Phi_{i, j, k}^{y y}+\Phi_{i, j, k}^{z z}+\Phi_{i, j, k}^{x y}-\Phi_{i, j, k}^{x z}\right] .
\end{aligned}
$$

Analogamente, discretizando (B.166) da célula de superfície na posição $(i, j, k)$, obtemos

$$
\begin{aligned}
& 2\left(\frac{u_{i+\frac{1}{2}, j, k}-u_{i-\frac{1}{2}, j, k}}{\delta x}\right)-2\left(\frac{v_{i, j+\frac{1}{2}, k}-v_{i, j-\frac{1}{2}, k}}{\delta y}\right) \\
& -\frac{1}{2}\left(\frac{u_{i+\frac{1}{2}, j, k+1}+u_{i-\frac{1}{2}, j, k+1}-u_{i+\frac{1}{2}, j, k}-u_{i-\frac{1}{2}, j, k}}{\delta z}\right. \\
& \left.+\frac{w_{i, j, k+\frac{1}{2}}+w_{i, j, k-\frac{1}{2}}-w_{i-1, j, k+\frac{1}{2}}-w_{i-1, j, k-\frac{1}{2}}}{\delta x}\right) \\
& -\frac{1}{2}\left(\frac{v_{i, j+\frac{1}{2}, k+1}+v_{i, j-\frac{1}{2}, k+1}-v_{i, j+\frac{1}{2}, k}-v_{i, j-\frac{1}{2}, k}}{\delta z}\right. \\
& \left.+\frac{w_{i, j+1, k+\frac{1}{2}}+w_{i, j+1, k-\frac{1}{2}}-w_{i, j, k+\frac{1}{2}}-w_{i, j, k-\frac{1}{2}}}{\delta y}\right) \\
& =\left[-\Phi_{i, j, k}^{x x}+\Phi_{i, j, k}^{y y}+\Phi_{i, j, k}^{x z}+\Phi_{i, j, k}^{y z}\right]
\end{aligned}
$$

a qual reescrevemos como

$$
\left(4+\frac{\delta x}{\delta z}\right) u_{i+\frac{1}{2}, j, k}+\left(4 \frac{\delta x}{\delta y}+\frac{\delta x}{\delta z}\right) v_{i, j-\frac{1}{2}, k}+\left(\frac{\delta x}{\delta y}-1\right) w_{i, j, k-\frac{1}{2}}=b_{1}
$$

onde

$$
\begin{aligned}
b_{1}= & 4 u_{i-\frac{1}{2}, j, k}+4 \frac{\delta x}{\delta y} v_{i, j+\frac{1}{2}, k}+\left(\frac{\delta x}{\delta z}\right)\left(u_{i+\frac{1}{2}, j, k+1}+u_{i-\frac{1}{2}, j, k+1}-u_{i-\frac{1}{2}, j, k}\right) \\
& +\left(w_{i, j, k+\frac{1}{2}}-w_{i-1, j, k+\frac{1}{2}}-w_{i-1, j, k-\frac{1}{2}}\right) \\
& +\left(\frac{\delta x}{\delta z}\right)\left(v_{i, j+\frac{1}{2}, k+1}+v_{i, j-\frac{1}{2}, k+1}-v_{i, j+\frac{1}{2}, k}\right) \\
& +\left(\frac{\delta x}{\delta y}\right)\left(w_{i, j+1, k+\frac{1}{2}}+w_{i, j+1, k-\frac{1}{2}}-w_{i, j, k+\frac{1}{2}}\right) \\
& +2 \delta x\left[-\Phi_{i, j, k}^{x x}+\Phi_{i, j, k}^{y y}+\Phi_{i, j, k}^{x z}+\Phi_{i, j, k}^{y z}\right] .
\end{aligned}
$$

As equações (B.169), (B.168) e (B.167) fornecem um sistema linear $(3 \times 3)$ para as velocidades $u_{i+\frac{1}{2}, j, k}, v_{i, j-\frac{1}{2}, k}, w_{i, j, k-\frac{1}{2}}$ que pode ser escrito na forma matricial como:

$$
\left[\begin{array}{ccc}
\left(4+\frac{\delta x}{\delta z}\right) & \left(4 \frac{\delta x}{\delta y}+\frac{\delta x}{\delta z}\right) & \left(\frac{\delta x}{\delta y}-1\right) \\
\left(\frac{\delta x}{\delta z}-\frac{\delta x}{\delta y}\right) & \left(4 \frac{\delta x}{\delta y}+1\right) & -\left(4 \frac{\delta x}{\delta z}+1\right) \\
1 & -\frac{\delta x}{\delta y} & -\frac{\delta x}{\delta z}
\end{array}\right]\left[\begin{array}{c}
u_{i+\frac{1}{2}, j, k} \\
v_{i, j-\frac{1}{2}, k} \\
w_{i, j, k-\frac{1}{2}}
\end{array}\right]=\left[\begin{array}{c}
b_{1} \\
b_{2} \\
b_{3}
\end{array}\right]
$$


Esse sistema pode ser resolvido facilmente pelo método de eliminação de Gauss. Com os valores de $u_{i+\frac{1}{2}, j, k}, v_{i, j-\frac{1}{2}, k}$ e $w_{i, j, k-\frac{1}{2}}$ calculados a pressão é obtida pela discretização de (B.163), no centro da célula de superfície, obtendo,

$$
\begin{aligned}
\tilde{p}_{i, j, k}= & \frac{1}{3 R e}\left[-\left(\frac{u_{i+\frac{1}{2}, j+1, k}+u_{i-\frac{1}{2}, j+1, k}-u_{i+\frac{1}{2}, j, k}-u_{i-\frac{1}{2}, j, k}}{\delta y}\right.\right. \\
& \left.+\frac{v_{i, j+\frac{1}{2}, k}+v_{i, j-\frac{1}{2}, k}-v_{i-1, j+\frac{1}{2}, k}-v_{i-1, j-\frac{1}{2}, k}}{\delta x}\right) \\
& -\left(\frac{u_{i+\frac{1}{2}, j, k+1}+u_{i-\frac{1}{2}, j, k+1}-u_{i+\frac{1}{2}, j, k}-u_{i-\frac{1}{2}, j, k}}{\delta z}\right. \\
& \left.+\frac{w_{i, j, k+\frac{1}{2}}+w_{i, j, k-\frac{1}{2}}-w_{i-1, j, k+\frac{1}{2}}-w_{i-1, j, k-\frac{1}{2}}}{\delta x}\right) \\
& +\left(\frac{v_{i, j+\frac{1}{2}, k+1}+v_{i, j-\frac{1}{2}, k+1}-v_{i, j+\frac{1}{2}, k}-v_{i, j-\frac{1}{2}, k}}{\delta z}\right. \\
& \left.\left.+\frac{w_{i, j+1, k+\frac{1}{2}}+w_{i, j+1, k-\frac{1}{2}}-w_{i, j, k+\frac{1}{2}}-w_{i, j, k-\frac{1}{2}}}{\delta y}\right)\right] \\
& +\frac{1}{3 R e}\left[\Phi_{i, j, k}^{x x}+\Phi_{i, j, k}^{y y}+\Phi_{i, j, k}^{z z}+2\left(-\Phi_{i, j, k}^{x y}-\Phi_{i, j, k}^{x z}+\Phi_{i, j, k}^{y z}\right)\right] .
\end{aligned}
$$

B.3.7 Células $\mathbf{S}$ possuem as faces $\left(i-\frac{1}{2}\right),\left(j-\frac{1}{2}\right)$ e $\left(k-\frac{1}{2}\right)$ em contato com células $\mathrm{E}$.

$$
\mathbf{n}=\left(-\frac{\sqrt{3}}{3},-\frac{\sqrt{3}}{3},-\frac{\sqrt{3}}{3}\right), \mathbf{m} \mathbf{1}=\left(\frac{\sqrt{2}}{2},-\frac{\sqrt{2}}{2}, 0\right), \mathbf{m} \mathbf{2}=\left(\frac{\sqrt{6}}{6}, \frac{\sqrt{6}}{6},-\frac{\sqrt{6}}{3}\right)
$$

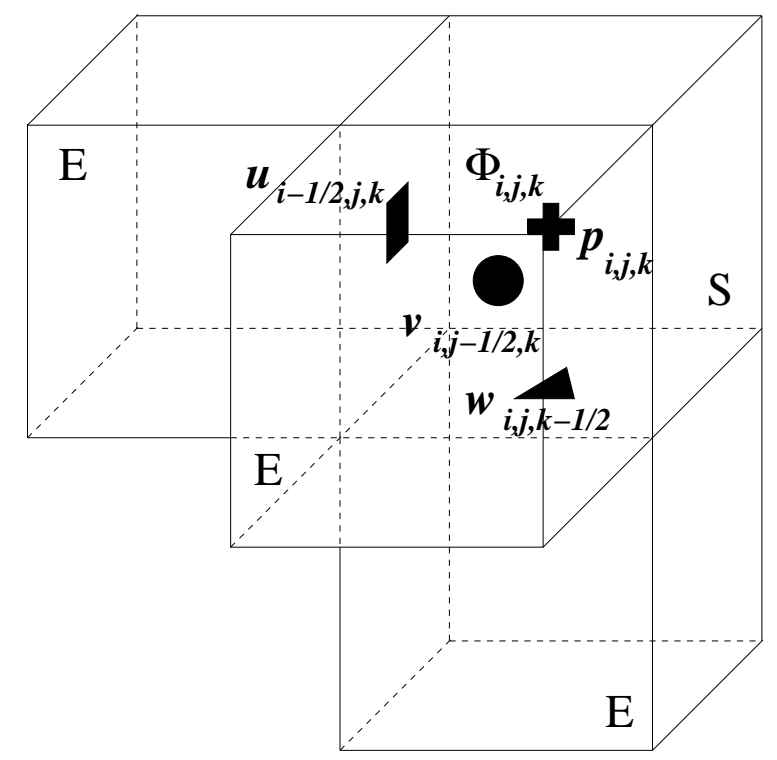

Figura B.23: Célula S com as faces $\left(i-\frac{1}{2}\right),\left(j-\frac{1}{2}\right)$ e $\left(k-\frac{1}{2}\right)$ em contato com faces de células vazias. 


$$
\begin{gathered}
\tilde{p}=\frac{1}{3 R e}\left[2\left(\frac{\partial u}{\partial y}+\frac{\partial v}{\partial x}\right)+2\left(\frac{\partial u}{\partial z}+\frac{\partial w}{\partial x}\right)+2\left(\frac{\partial v}{\partial z}+\frac{\partial w}{\partial y}\right)\right. \\
\left.+\Phi^{x x}+\Phi^{y y}+\Phi^{z z}+2\left(\Phi^{x y}+\Phi^{x z}+\Phi^{y z}\right)\right] \\
-2 \frac{\partial u}{\partial x}+2 \frac{\partial v}{\partial y}-\left(\frac{\partial u}{\partial z}+\frac{\partial w}{\partial x}\right)+\left(\frac{\partial v}{\partial z}+\frac{\partial w}{\partial y}\right)=-\left[-\Phi^{x x}+\Phi^{y y}-\Phi^{x z}+\Phi^{y z}\right], \\
-2 \frac{\partial u}{\partial x}-2 \frac{\partial v}{\partial y}+4 \frac{\partial w}{\partial z}-2\left(\frac{\partial u}{\partial y}+\frac{\partial v}{\partial x}\right)+\left(\frac{\partial u}{\partial z}+\frac{\partial w}{\partial x}\right)+\left(\frac{\partial v}{\partial z}+\frac{\partial w}{\partial y}\right) \\
=-\left[-\Phi^{x x}-\Phi^{y y}+2 \Phi^{z z}-2 \Phi^{x y}+\Phi^{x z}+\Phi^{y z}\right],
\end{gathered}
$$

Agora, adicionando as equações (B.173) e (B.174) obtém-se

$$
-2 \frac{\partial u}{\partial x}+2 \frac{\partial w}{\partial z}-\left(\frac{\partial u}{\partial y}+\frac{\partial v}{\partial x}\right)+\left(\frac{\partial v}{\partial z}+\frac{\partial w}{\partial y}\right)=\left[\Phi^{x x}-\Phi^{z z}+\Phi^{x y}-\Phi^{y z}\right]
$$

Pela discretização da equação da continuidade obtemos

$$
-u_{i-\frac{1}{2}, j, k}-\frac{\delta x}{\delta y} v_{i, j-\frac{1}{2}, k}-\frac{\delta x}{\delta z} w_{i, j, k-\frac{1}{2}}=b_{3}
$$

onde

$$
b_{3}=-u_{i+\frac{1}{2}, j, k}-\frac{\delta x}{\delta y} v_{i, j+\frac{1}{2}, k}-\frac{\delta x}{\delta z} w_{i, j, k+\frac{1}{2}} .
$$

Agora, equação (B.173) aplicada no centro da célula fornece

$$
\begin{aligned}
& -2\left(\frac{u_{i+\frac{1}{2}, j, k}-u_{i-\frac{1}{2}, j, k}}{\delta x}\right)+2\left(\frac{v_{i, j+\frac{1}{2}, k}-v_{i, j-\frac{1}{2}, k}}{\delta y}\right) \\
& -\frac{1}{2}\left(\frac{u_{i+\frac{1}{2}, j, k+1}+u_{i-\frac{1}{2}, j, k+1}-u_{i+\frac{1}{2}, j, k}-u_{i-\frac{1}{2}, j, k}}{\delta z}\right. \\
& \left.+\frac{w_{i+1, j, k+\frac{1}{2}}+w_{i+1, j, k-\frac{1}{2}}-w_{i, j, k+\frac{1}{2}}-w_{i, j, k-\frac{1}{2}}}{\delta x}\right) \\
& +\frac{1}{2}\left(\frac{v_{i, j+\frac{1}{2}, k+1}+v_{i, j-\frac{1}{2}, k+1}-v_{i, j+\frac{1}{2}, k}-v_{i, j-\frac{1}{2}, k}}{\delta z}\right. \\
& \left.+\frac{w_{i, j+1, k+\frac{1}{2}}+w_{i, j+1, k-\frac{1}{2}}-w_{i, j, k+\frac{1}{2}}-w_{i, j, k-\frac{1}{2}}}{\delta y}\right) \\
& =-\left[-\Phi_{i, j, k}^{x x}+\Phi_{i, j, k}^{y y}-\Phi_{i, j, k}^{x z}+\Phi_{i, j, k}^{y z}\right]
\end{aligned}
$$

que pode ser reescrita como (multiplicando por 2 e por $\delta x$ )

$$
\left(4+\frac{\delta x}{\delta z}\right) u_{i-\frac{1}{2}, j, k}-\left(4 \frac{\delta x}{\delta y}+\frac{\delta x}{\delta z}\right) v_{i, j-\frac{1}{2}, k}+\left(1-\frac{\delta x}{\delta y}\right) w_{i, j, k-\frac{1}{2}}=b_{2}
$$


onde

$$
\begin{aligned}
b_{2}= & 4 u_{i+\frac{1}{2}, j, k}-4 \frac{\delta x}{\delta y} v_{i, j+\frac{1}{2}, k}+\left(\frac{\delta x}{\delta z}\right)\left(u_{i+\frac{1}{2}, j, k+1}+u_{i-\frac{1}{2}, j, k+1}-u_{i+\frac{1}{2}, j, k}\right) \\
& +\left(w_{i+1, j, k+\frac{1}{2}}+w_{i+1, j, k-\frac{1}{2}}-w_{i, j, k+\frac{1}{2}}\right) \\
& -\left(\frac{\delta x}{\delta z}\right)\left(v_{i, j+\frac{1}{2}, k+1}+v_{i, j-\frac{1}{2}, k+1}-v_{i, j+\frac{1}{2}, k}\right) \\
& -\left(\frac{\delta x}{\delta y}\right)\left(w_{i, j+1, k+\frac{1}{2}}+w_{i, j+1, k-\frac{1}{2}}-w_{i, j, k+\frac{1}{2}}\right) \\
& -2 \delta x\left[-\Phi_{i, j, k}^{x x}+\Phi_{i, j, k}^{y y}-\Phi_{i, j, k}^{x z}+\Phi_{i, j, k}^{y z}\right] .
\end{aligned}
$$

Analogamente, discretizando (B.175) da célula de superfície na posição $(i, j, k)$, obtemos

$$
\begin{aligned}
& -2\left(\frac{u_{i+\frac{1}{2}, j, k}-u_{i-\frac{1}{2}, j, k}}{\delta x}\right)+2\left(\frac{w_{i, j, k+\frac{1}{2}}-w_{i, j, k-\frac{1}{2}}}{\delta z}\right) \\
& -\frac{1}{2}\left(\frac{u_{i+\frac{1}{2}, j+1, k}+u_{i-\frac{1}{2}, j+1, k}-u_{i+\frac{1}{2}, j, k}-u_{i-\frac{1}{2}, j, k}}{\delta y}\right. \\
& \left.+\frac{v_{i+1, j+\frac{1}{2}, k}+v_{i+1, j-\frac{1}{2}, k}-v_{i, j+\frac{1}{2}, k}-v_{i, j-\frac{1}{2}, k}}{\delta x}\right) \\
& +\frac{1}{2}\left(\frac{v_{i, j+\frac{1}{2}, k+1}+v_{i, j-\frac{1}{2}, k+1}-v_{i, j+\frac{1}{2}, k}-v_{i, j-\frac{1}{2}, k}}{\delta z}\right. \\
& \left.+\frac{w_{i, j+1, k+\frac{1}{2}}+w_{i, j+1, k-\frac{1}{2}}-w_{i, j, k+\frac{1}{2}}-w_{i, j, k-\frac{1}{2}}}{\delta y}\right) \\
& =\left[\Phi_{i, j, k}^{x x}-\Phi_{i, j, k}^{z z}+\Phi_{i, j, k}^{x y}-\Phi_{i, j, k}^{y z}\right]
\end{aligned}
$$

a qual reescrevemos como

$$
\left(4+\frac{\delta x}{\delta y}\right) u_{i-\frac{1}{2}, j, k}+\left(1-\frac{\delta x}{\delta z}\right) v_{i, j-\frac{1}{2}, k}-\left(4 \frac{\delta x}{\delta z}+\frac{\delta x}{\delta y}\right) w_{i, j, k-\frac{1}{2}}=b_{1}
$$

onde

$$
\begin{aligned}
b_{1}= & 4 u_{i+\frac{1}{2}, j, k}-4 \frac{\delta x}{\delta z} w_{i, j, k+\frac{1}{2}}+\left(\frac{\delta x}{\delta y}\right)\left(u_{i+\frac{1}{2}, j+1, k}+u_{i-\frac{1}{2}, j+1, k}-u_{i+\frac{1}{2}, j, k}\right) \\
& +\left(v_{i+1, j+\frac{1}{2}, k}+v_{i+1, j-\frac{1}{2}, k}-v_{i, j+\frac{1}{2}, k}\right) \\
& -\left(\frac{\delta x}{\delta z}\right)\left(v_{i, j+\frac{1}{2}, k+1}+v_{i, j-\frac{1}{2}, k+1}-v_{i, j+\frac{1}{2}, k}\right) \\
& -\left(\frac{\delta x}{\delta y}\right)\left(w_{i, j+1, k+\frac{1}{2}}+w_{i, j+1, k-\frac{1}{2}}-w_{i, j, k+\frac{1}{2}}\right) \\
& +2 \delta x\left[\Phi_{i, j, k}^{x x}-\Phi_{i, j, k}^{z z}+\Phi_{i, j, k}^{x y}-\Phi_{i, j, k}^{y z}\right] .
\end{aligned}
$$

As equações (B.178), (B.177) e (B.176) fornecem um sistema linear $(3 \times 3)$ para as velocidades $u_{i-\frac{1}{2}, j, k}, v_{i, j-\frac{1}{2}, k}, w_{i, j, k-\frac{1}{2}}$ que pode ser escrito na forma matricial como:

$$
\left[\begin{array}{ccc}
\left(4+\frac{\delta x}{\delta z}\right) & \left(1-\frac{\delta x}{\delta z}\right) & -\left(4 \frac{\delta x}{\delta z}+\frac{\delta x}{\delta y}\right) \\
\left(4+\frac{\delta x}{\delta z}\right) & -\left(4 \frac{\delta x}{\delta y}+\frac{\delta x}{\delta z}\right) & \left(1-\frac{\delta x}{\delta y}\right) \\
-1 & -\frac{\delta x}{\delta y} & -\frac{\delta x}{\delta z}
\end{array}\right]\left[\begin{array}{c}
u_{i-\frac{1}{2}, j, k} \\
v_{i, j-\frac{1}{2}, k} \\
w_{i, j, k-\frac{1}{2}}
\end{array}\right]=\left[\begin{array}{l}
b_{1} \\
b_{2} \\
b_{3}
\end{array}\right]
$$


Esse sistema pode ser resolvido facilmente pelo método de eliminação de Gauss. Com os valores de $u_{i-\frac{1}{2}, j, k}, v_{i, j-\frac{1}{2}, k}$ e $w_{i, j, k-\frac{1}{2}}$ calculados a pressão é obtida pela discretização de (B.172), no centro da célula de superfície, obtendo,

$$
\begin{aligned}
\tilde{p}_{i, j, k}= & \frac{1}{3 R e}\left[\left(\frac{u_{i+\frac{1}{2}, j+1, k}+u_{i-\frac{1}{2}, j+1, k}-u_{i+\frac{1}{2}, j, k}-u_{i-\frac{1}{2}, j, k}}{\delta y}\right.\right. \\
& \left.+\frac{v_{i+1, j+\frac{1}{2}, k}+v_{i+1, j-\frac{1}{2}, k}-v_{i, j+\frac{1}{2}, k}-v_{i, j-\frac{1}{2}, k}}{\delta x}\right) \\
& +\left(\frac{u_{i+\frac{1}{2}, j, k+1}+u_{i-\frac{1}{2}, j, k+1}-u_{i+\frac{1}{2}, j, k}-u_{i-\frac{1}{2}, j, k}}{\delta z}\right. \\
& \left.+\frac{w_{i+1, j, k+\frac{1}{2}}+w_{i+1, j, k-\frac{1}{2}}-w_{i, j, k+\frac{1}{2}}-w_{i, j, k-\frac{1}{2}}}{\delta x}\right) \\
& +\left(\frac{v_{i, j+\frac{1}{2}, k+1}+v_{i, j-\frac{1}{2}, k+1}-v_{i, j+\frac{1}{2}, k}-v_{i, j-\frac{1}{2}, k}}{\delta z}\right. \\
& \left.\left.+\frac{w_{i, j+1, k+\frac{1}{2}}+w_{i, j+1, k-\frac{1}{2}}-w_{i, j, k+\frac{1}{2}}-w_{i, j, k-\frac{1}{2}}}{\delta y}\right)\right] \\
& +\frac{1}{3 R e}\left[\Phi_{i, j, k}^{x x}+\Phi_{i, j, k}^{y y}+\Phi_{i, j, k}^{z z}+2\left(\Phi_{i, j, k}^{x y}+\Phi_{i, j, k}^{x z}+\Phi_{i, j, k}^{y z}\right)\right] .
\end{aligned}
$$

